\title{
Synthesis of chondroitin/dermatan sulfate-like oligosaccharides and evaluation of their protein affinity by fluorescence polarization
}

Susana Maza, M. Mar Kayser, Giuseppe Macchione, Javier López-Prados, Jesús Angulo, José L. de Paz, * Pedro M. Nieto*

Glycosystems Laboratory, Instituto de Investigaciones Químicas (IIQ), Centro de Investigaciones Científicas Isla de La Cartuja, CSIC and Universidad de Sevilla, Americo Vespucio, 49, 41092 Sevilla, Spain.

* Corresponding author. Fax: +34 954 460565; e-mail: jlpaz@iiq.csic.es

\begin{abstract}
Here, we present a novel approach for the chemical synthesis of chondroitin and dermatan sulfate oligosaccharides. A key point of this strategy is the preparation and use of an $\mathrm{N}$-trifluoroacetyl galactosamine building block containing a 4,6-O-di-tertbutylsilylene group. Glycosylation reactions proceeded in good yields (74-91\%) with our protecting group distribution. Using this approach, we have synthesized, for the first time, a chondroitin/dermatan sulfate-like tetrasaccharide that contains both types of uronic acids, D-glucuronic and L-iduronic acid. Moreover, we have employed a fluorescence polarization competition assay to evaluate the interactions between the synthesized oligosaccharides and FGF-2 (basic Fibroblast Growth Factor). Our results show that this method, using standard instrumentation and minimal sample consumption, is a powerful tool for the rapid analysis of the glycosaminoglycan affinity for proteins in solution.

\section{Introduction}


Chondroitin sulfate (CS) and dermatan sulfate (DS) are highly heterogeneous and sulfated, linear polysaccharides that belong to the glycosaminoglycan (GAG) family., ${ }^{1,2}$ $\mathrm{CS}$ is formed by the repetition of disaccharide units of D-glucuronic acid (GlcA) and $\mathrm{N}$ acetyl-D-galactosamine (GalNAc), following the sequence GlcA- $\beta(1 \rightarrow 3)$-GalNAc$\beta(1 \rightarrow 4)$. The disaccharide repeating unit of the structurally related DS mainly contains L-iduronic acid (IdoA) in place of GlcA. Both polysaccharides may contain sulfate groups at different positions of the chain (Figure 1). These sulfate groups are introduced during the biosynthesis of these polymers, through the action of specific sulfotransferases, giving rise to GAG chains with a high level of structural diversity. The microheterogeneity of CS and DS can be considered as a capacity to encode information and control a wide variety of biological processes by specific interactions with certain proteins. ${ }^{3}$ As in the case of others members of the GAG family, such as heparin and heparan sulfate, ${ }^{4-8}$ it is proposed that defined CS and DS oligosaccharide sequences are responsible for specific protein recognition and subsequent activity. ${ }^{3}$ However, little is known about the exact structural requirements for these interactions. In this context, synthetic CS and DS oligosaccharides ${ }^{9-13}$ are useful tools for the establishment of structure-activity relationships and the preparation of mimetics that potentially modulate the biological functions of the natural products. ${ }^{14-16}$ Interestingly, CS and DS are often found as co-polymeric structures. ${ }^{17}$ These hybrid CS/DS chains, containing both types of uronic acids, GlcA and IdoA, are involved in growth factor signalling and neuronal growth and development. ${ }^{17}$ The interaction between CS/DS and several chemokines and growth factors, including FGF-2 (basic Fibroblast Growth Factor), is mediated by oversulfated oligosaccharide sequences containing GlcA/IdoA-GalNAc $\left(4,6-\mathrm{OSO}_{3}\right) .{ }^{18-21}$ In order to study these interactions at the molecular level, it would be very useful the access to well-defined synthetic CS/DS 
oligosaccharides. However, to the best of our knowledge, the synthesis of a CS/DS oligomer, containing both GlcA and IdoA, has not yet been reported.

Despite significant advances in the field, ${ }^{22-33}$ the synthesis of GAG oligosaccharides, including DS and CS, is still challenging, mainly due to the low reactivity of the building blocks required. ${ }^{34-37}$ There is still a great demand for efficient synthetic strategies, involving a robust and reliable set of carbohydrate building blocks. This is an important point for the successful automation of oligosaccharide synthesis..$^{33,38-40}$ Here, we present a novel approach for the synthesis of CS and DS oligomers that is based on the use of an $N$-trifluroacetyl-protected galactosamine building block. Glycosylation reactions proceeded in high yields using our design of protecting groups. The efficiency of this strategy is illustrated with the total synthesis of the oversulfated tetrasaccharide $\mathbf{1}$ (Scheme 1) that contains both IdoA and GlcA and bears sulfate groups at positions 4 and 6 of the GalNAc units, position 2 of the uronic acid moieties, and position 4 of the non-reducing terminus. All these positions may be sulfated in the natural products (Figure 1), except position 4 of the non-reducing end. Interestingly, the introduction of a "non-natural" sulfate group at the reducing end did not significantly affect the FGF-2 affinity of a synthetic heparin hexasaccharide ${ }^{41}$ and an "artificial" IdoA monosaccharide, sulfated at positions 2 and 4, showed considerable binding to several proteins. ${ }^{42,43}$ Moreover, we have developed a fluorescence polarization assay to analyse the binding of tetrasaccharide $\mathbf{1}$ and its di- $O$-benzylated precursor to a model heparin-binding protein, FGF-2. Fluorescence polarization measurements allowed the study of GAG oligosaccharide-protein interactions in solution, with minimal time and sample consumption, and using a standard fluorescence reader. Therefore, our results indicate that this method can be considered as a powerful tool to evaluate the binding 
affinities of synthetic oligosaccharides for receptors of biological relevance, helping to establish structure-activity relationships.

\section{Results and discussion}

\section{Synthesis of chondroitin/dermatan sulfate-like oligosaccharides}

For the synthesis of the CS/DS-related tetrasaccharide 1, we first prepared the required monosaccharide building blocks 2-4 (Scheme 1). Glucuronic acid trichloroacetimidate 2 was prepared from known diol $\mathbf{5},{ }^{44}$ as shown in Scheme 2. Selective oxidation at position 6 was performed by treatment of the diol 5 with calcium hypochlorite and catalytic TEMPO, under phase-transfer conditions. ${ }^{44}$ Strict control of the reaction time and temperature, and quenching with $\mathrm{Na}_{2} \mathrm{SO}_{3}$ were required to avoid the chlorination of the electron-rich 4-methoxyphenyl ring. ${ }^{45}$ The carboxylate intermediate was then esterified with $\mathrm{BnBr}$ and $\mathrm{Bu}_{4} \mathrm{NI}$ in $\mathrm{DMF}$ at $60^{\circ} \mathrm{C}$ to give the benzyl uronate 6 . Levulinoylation at position 4 , followed by oxidative removal of the 4-methoxyphenyl group with CAN, and trichloroacetimidate formation, afforded glycosyl donor 2. Starting from diol 9, ${ }^{46,47}$ iduronic acid trichloroacetimidate $\mathbf{3}$ was obtained by levulinoylation, selective desilylation and anomeric activation with $\mathrm{Cl}_{3} \mathrm{CCN}$ and $\mathrm{K}_{2} \mathrm{CO}_{3}$. Regarding the galactosamine unit, two types of building blocks, possessing different protections on the amino group, have been employed, up to date, for the synthesis of CS and DS oligosaccharides. ${ }^{10}$ 2-Azido-2-deoxy-galactose derivatives ${ }^{11,12,48-51}$ present some limitations for their general use due to the non-participating character of the azido moiety. On the contrary, 2-deoxy-2-trichloroacetamido-galactose building blocks lead to the stereoselective formation of the required 1,2-trans glycosidic bond. Impressive synthesis of CS oligomers have been reported using $N$-trichloroacetyl(TCA)-protected units. ${ }^{3,32,52-55}$ However, this amino protecting group is associated with some problems. For example, we $\mathrm{e}^{56}$ and others ${ }^{57}$ have detected the formation of stable trichlorooxazoline 
side products during glycosidation of 2-deoxy-2-trichloroacetamido donors. Moreover, several difficulties are occasionally encountered in the final transformation to the desired 2-acetamido group. Thus, the deprotection of multiple $N$-TCA groups by basic hydrolysis, followed by selective $N$-acetylation, requires very long reaction times. ${ }^{57}$ Alternatively, radical reduction using tributylstannane afford, in some cases, significant amounts of mono- and dichloroacetamide intermediates ${ }^{58,59}$ For these reasons, we considered the use of an alternative amine-protecting group for the synthesis of CS/DS oligosaccharides. We chose an $N$-trifluoroacetyl (TFA) group because it can be easily removed under mild conditions while ensures high $\beta$ selectivities in glycosylation reactions. ${ }^{60}$

Thus, we first planned the preparation of a $N$-TFA-protected galactosamine unit that should act as an efficient glycosyl acceptor in coupling reactions with uronic acid donors. The synthesis of such a compound was challenging (Scheme 3). Known tetraacetate $12^{61,62}$ was synthesized from galactosamine hydrochloride in $66 \%$ yield by treatment with $\mathrm{NaOMe}$ and then with trifluoroacetic anhydride and $\mathrm{Et}_{3} \mathrm{~N}$ in $\mathrm{MeOH}$, followed by extensive acetylation $\left(\mathrm{Ac}_{2} \mathrm{O}, \mathrm{Py}, \mathrm{DMAP}\right)$. Compound 12 was transformed into 4-methoxyphenyl glycoside 15 by glycosylation with 4-methoxyphenol, followed by de- $O$-acetylation and benzylidenation. This compound was an ideal candidate for our synthetic approach because the 4,6-O-benzylidene acetal would allow the selective sulfation of these positions at the end of the synthesis to generate, among others, biologically relevant type E sulfation sequences. ${ }^{3}$ However, 15 suffered from poor solubility in organic solvents such as dichloromethane, toluene and acetonitrile, and glycosylation attempts with uronic acid donor $2^{63}$ in THF or $\mathrm{THF} / \mathrm{CH}_{2} \mathrm{Cl}_{2}$ mixtures did not give any desired disaccharide. We then decided to prepare compound $\mathbf{1 7}$ with a silyl ether group at the anomeric position. Selective cleavage of the anomeric acetyl group of 
12 was followed by treatment with TDSCl to afford derivative 16. Acetate hydrolysis and benzylidenation gave compound 17. Surprisingly, this derivative proved to be unstable during silica gel chromatography. Therefore, $\mathbf{1 7}$ was not considered anymore as building block for our synthetic scheme. We then directed our attention to the 6-Ochloroacetylated building block $\mathbf{1 8}$, efficiently synthetized from $\mathbf{1 6}$ in two steps. It was anticipated that diol 18 would be selectively glycosylated at position 3. Unfortunately, glycosylation reactions between diol $\mathbf{1 8}$ and uronic acid donor $2^{63}$ proceeded in low yield $(<15 \%)$ and regioselectivity. Finally, we decided to prepare monosaccharide 4 containing a di-tert-butylsilylene group. It has been reported that this protecting group, compared to benzylidene acetals, confers desirable properties to glycosyl acceptors, such as better solubility in most organic solvents and higher stability under acidic glycosylation conditions. ${ }^{58,64}$ Thus, we treated monosaccharide $\mathbf{1 4}$ with di-tertbutylsilyl bistriflate in pyridine to obtain 4 in high yield. Gratifyingly, coupling of $\mathbf{4}$ with glucuronic acid trichloroacetimidate 2 gave the desired $\beta(1 \rightarrow 3)$ disaccharide 19 in excellent yield (Scheme 4). These results highlight the profound effect that protecting group distribution of the building blocks has on glycosylation reactions. ${ }^{65} \mathrm{We}$ also perfomed the glycosylation reaction between $\mathbf{4}$ and iduronic acid donor $\mathbf{3}$. The target $\alpha$ $(1 \rightarrow 3)$ disaccharide $\mathbf{2 0}$ was also obtained in high yield. The small-to-zero coupling constants for IdoA protons indicated that this residue mainly exists in ${ }^{1} \mathrm{C}_{4}$ conformation, and the value of the ${ }^{1} \mathrm{~J}_{\mathrm{C}, \mathrm{H}}(172 \mathrm{~Hz})$ confirmed the $\alpha$ configuration of the new glycosidic linkage. ${ }^{66}$

Next, we studied the $2+2$ assembly of the disaccharide units 19 and 20 to generate a tetrasaccharide sequence containing both GlcA and IdoA. The 4,6-di-tert-butylsilylene group $^{67}$ of galactose and galactosamine donors leads to the selective formation of $\alpha$ glycosides despite the presence of participating groups at position $2 .{ }^{68}$ In some cases, 
even 4,6-O-benzylidene derivatives of galactosamine donors may lead to loss of stereocontrol in coupling reactions with glucuronic acid-derived acceptors. ${ }^{69}$ For these reasons, we decided to transform disaccharide $\mathbf{2 0}$ in a suitable protected donor containing less sterically hindered acetyl groups at positions 4 and 6 to obtain the desired 1,2-trans glycoside with excellent stereoselectivity. Cleavage of the silylene group gave diol 21. The isolation of this compound was tricky because $\mathbf{2 1}$ formed gels in several solvents, such as toluene, $\mathrm{CH}_{2} \mathrm{Cl}_{2}$ and EtOAc. Therefore, diol 21 was directly acetylated, without further purification, to yield compound 22. Removal of the 4methoxyphenyl group followed by treatment with $\mathrm{Cl}_{3} \mathrm{CCN}$ and catalytic DBU gave donor 24. On the other hand, 19 was transformed into acceptor 25 by treatment with hydrazine monohydrate in a pyridine/acetic acid solution. Coupling of disaccharides $\mathbf{2 4}$ and $\mathbf{2 5}$ gave the target $\beta$ tetrasaccharide $\mathbf{2 6}$ in high yield. This result demonstrates the utility of our synthetic route for the assembly of CS and DS oligosaccharides, paving the way for other sequences with different sulfation patterns.

Tetrasaccharide $\mathbf{2 6}$ was submitted to the deprotection/sulfation steps to obtain final compound 1. Cleavage of the silylene group was performed by treatment with $(\mathrm{HF})_{\mathrm{n}} \cdot \mathrm{Py}$ complex. Hydrolysis of the acyl groups, benzyl and methyl esters, and trifluoroacetamides was carried out by treatment with lithium hydroperoxide and then $\mathrm{NaOH}$ to give compound 28. The amine groups were selectively acetylated with $\mathrm{Ac}_{2} \mathrm{O}$ in $\mathrm{MeOH}$ to provide intermediate $\mathbf{2 9}$, which was purified by gel permeation chromatography. Then, extensive $O$-sulfation using $\mathrm{SO}_{3} \cdot \mathrm{Me}_{3} \mathrm{~N}$ in $\mathrm{DMF}$ at $100{ }^{\circ} \mathrm{C}$ under microwave heating ${ }^{41,70}$ gave cleanly the corresponding hepta- $O$-sulfated tetrasaccharide 30, which proved to be soluble in water. This compound was converted into the corresponding calcium salt for NMR characterization because the ${ }^{1} \mathrm{H}-\mathrm{NMR}$ spectrum of the sodium salt showed considerable signal overlap. NMR spectra showed the 
characteristic downfield shifts of the proton and carbon signals at positions bearing a sulfate group (see Table 1 and 2). COSY, HSQC and TOCSY NMR experiments were employed for the structural assignment. Additionally, mass spectroscopic analysis confirmed the structure of $\mathbf{3 0}$. Finally, hydrogenolysis of $\mathbf{3 0}$ gave the fully deprotected tetrasaccharide $\mathbf{1}$ in good yield. The structure of $\mathbf{1}$ was confirmed by NMR and mass spectroscopic analysis. The values of ${ }^{1} \mathrm{H}$ and ${ }^{13} \mathrm{C}$ NMR chemical shifts for sulfated positions are in good agreement with those reported in the literature for similar GAG sulfated sequences. ${ }^{41,53}$

\section{Fluorescence polarization measurements}

Fluorescent polarization is a powerful tool for the study of biomolecular interactions in solution. ${ }^{71}$ It is based on the observation that when a fluorescent molecule is excited with plane-polarized light, the remaining polarization of the emitted light depends on the rotational rate of the fluorescent molecule in solution that is inversely related to its molecular weight. Thus, the light emitted by a small fluorescent compound, which rotates quickly in solution, is highly depolarized and, therefore, the polarization value is low. If the fluorescent probe binds to a high molecular weight molecule, for example, a protein, the large complex rotates slower in solution, the emitted light remains polarized, and the polarization value is higher. Importantly, fluorescence polarization measurements do not require immobilization of protein or ligand to a surface for the analysis of the interaction. Thus, ligand's bound/free ratio can be directly measured in solution, avoiding potential misleading effects derived from the attachment of the biomolecules to solid supports. Moreover, this technique is adequate for high throughput screening, requires very little amount of samples, and is well suited for the binding analysis of small ligands, such as oligosaccharides, to a protein receptor. Despite these advantages, fluorescence polarization has had a limited use in the study of 
carbohydrate-protein interactions, ${ }^{72,73}$ in part due to the lack of sensitive and appropiate instrumentation until the late 90's. Interestingly, competition assays can be easily designed to study the binding affinities of nonfluorescent ligands. ${ }^{72}$ In fact, we employed a competition experiment to evaluate the affinity of synthetic tetrasaccharides $\mathbf{1}$ and $\mathbf{3 0}$ to FGF-2, as described below.

First, we prepared five different fluorescein-conjugated glycosaminoglycan oligosaccharides (36-40) to select an optimal probe for binding studies with FGF-2 (Scheme 7 and Supplementary information). Commercially available oligosaccharides 31-35, derived from natural heparin by enzymatic depolimerization, were functionalized by reaction of the aldehyde group of the reducing end of the chain with a hydrazidecontaining fluorescein molecule. ${ }^{74}$ The corresponding glycosyl hydrazides were obtained in good yield, after purification by reverse phase C-18 chromatography. Then, fluorescent labelled sugars 36-40 were mixed with a fixed concentration of FGF-2, and polarization was measured in 384-well microplates using a standard fluorescence microplate reader (Figure 2). Control wells containing only the fluorescent probe, without any protein, were included in the experiment. As expected, ${ }^{75,76}$ heparin hexasaccharide $\mathbf{3 8}$ and tetrasaccharide $\mathbf{3 7}$ bound to FGF-2 since a significant increased polarization value was observed in FGF-2 containing wells. No interaction was detected for heparin disaccharide 36 and hyaluronic acid oligosaccharides 39 and 40, ruling out any binding of FGF-2 to the fluorescein tag. Hexa 38, which gave best binding, was chosen as optimal probe for inhibition experiments (see below). Importantly, the use of 384-well plates allowed the minimization of the sample quantities required for these experiments: the standard assay was performed with $10 \mathrm{nM}$ fluorescent probe and approximately $100 \mathrm{nM}$ protein in a final volume of $40 \mu \mathrm{L}$ per well. 
Next, the binding of $\mathbf{3 8}$ to FGF-2 was measured with increasing concentrations of protein (see Supplementary information, Figure S1), giving the corresponding binding curve that was analyzed as a Langmuir isotherm to determine the dissociation constant $\left(\mathrm{K}_{\mathrm{D}}\right)$. The obtained value $(117 \pm 10 \mathrm{nM})$ was consistent with a previous measurement, in solution, of the binding affinity between a similar heparin hexasaccharide and FGF$2 .^{75}$

A competition binding assay was then optimized to analyze the binding affinites of nonfluorescent ligands, such as tetrasaccharides $\mathbf{1}$ and 30, to FGF-2. Thus, the polarization of samples containing fixed concentrations of protein and fluorescent probe were recorded in the presence of a certain concentration of potential competitors (Figure 3). In these experiments, we chose an FGF-2 concentration close to the $K_{D}$ of the interaction with $\mathbf{3 8}$ in order to get a high enough polarization value while still using the minimal amount of inhibitor. Besides 1 and 30, synthetic oligosaccharides $\mathbf{4 1 - 4 6}{ }^{41,77}$ were included in the screening (Figure 4). The displacement of fluorescent $\mathbf{3 8}$ by an active competitor resulted in a decrease of the polarization value (Figures 3 and 4). In this way, the inhibitory capacity of non labelled compounds could be easily and quickly screened. As shown in Figure 4, at $25 \mu \mathrm{M}$ concentration, monosaccharide $\mathbf{4 1}$ and disaccharides $\mathbf{4 2}$ and $\mathbf{4 6}$ did not significantly affect the interaction between fluorescent 38 and FGF-2, while hexasaccharides $\mathbf{4 4}$ and $\mathbf{4 5}$ strongly inhibited the binding. Interestingly, the presence of $25 \mu \mathrm{M}$ of $\mathbf{1}$ and $\mathbf{3 0}$ gave $63-67 \%$ inhibition. A similar effect was observed with tetrasaccharide 43. These results indicated that CS/DS-related tetrasaccharides $\mathbf{1}$ and $\mathbf{3 0}$ are able to interact with FGF-2 and that their relative inhibitory potencies are similar to the one displayed by a heparin tetrasaccharide. After demonstrating that this fluorescence polarization assay can be used for the rapid screening of a library of compounds, we studied the inhibitory potency of oversulfated 1 
in more detail. We measured the polarization of samples containing FGF-2, fluorescent 38 and increasing concentrations of tetrasaccharide 1 (Figure 5). The obtained curve was fitted to the equation for a simple one-site competitive interaction. An $\mathrm{IC}_{50}$ value of $15 \mu \mathrm{M}$ was estimated for compound $\mathbf{1}$. In terms of screening for inhibitors, our fluorescence polarization competition assay is advantageous over methods that require the immobilization of the ligand or the protein on a solid support and are based on the inhibition of the interaction that occurs at the surface, because this surface interaction can be affected by multivalency, involves unknown amounts of one of the biomolecules and requires additional washing and incubation steps.

The binding of FGF-2 to cell surface heparan sulfate GAGs is essential for tumor angiogenesis and growth. Inhibition of angiogenesis is a well established and important anti-cancer strategy and, therefore, there is a great interest on compounds that potentially inhibit the FGF-2/GAG interaction. ${ }^{78}$ Our data indicate that CS/DS tetrasaccharides $\mathbf{1}$ and $\mathbf{3 0}$ display considerable inhibitory activity and can be considered as starting points for the design and synthesis of more active compounds. Moreover, recent studies ${ }^{78,79}$ indicate that the introduction of lipophilic groups on the structure of synthetic antiangiogenic molecules improved the properties of these anticancer agents, and, in this context, the activity showed by the di-O-benzylated $\mathbf{3 0}$ is particularly remarkable.

\section{Conclusions}

We have prepared a $N$-TFA-protected galactosamine unit as key building block for the synthesis of CS and DS oligosaccharides. While the participating $N$-TFA group ensures the desired 1,2-trans stereochemistry of the glycosidic bond, the temporary introduction of a di-tert-butylsilylene group at positions 4 and 6 transforms this moiety in an excellent glycosyl acceptor for coupling reactions with uronic acid donors. Our results 
provide an additional illustration of the profound impact that protecting groups have on the success of a glycosylation reaction. Moreover, our protecting group design is compatible with the deprotection/sulfation steps required for the preparation of final CS and DS sequences. Following this strategy, oversulfated CS/DS-like tetrasaccharide 1 was successfully synthesized. Importantly, our approach can be easily applied to the synthesis of other CS and DS oligosaccharides, bearing different sulfate patterns. On the other hand, we have developed a fluorescence polarization assay for the rapid screening of the interactions between the synthesized oligosaccharides and proteins. The binding is analysed in solution, avoiding the potential artefacts and the additional washing steps that are typically associated to assays where the receptor or the ligand is immobilized on a solid surface. The only requirement to perform these experiments is the preparation of an adequate fluorescent probe. With this probe at hand, we could evaluate the relative binding affinities of a small library of non-fluorescent synthetic GAG oligosaccharides, including synthesized CS/DS tetrasaccharides $\mathbf{1}$ and $\mathbf{3 0}$, to a model heparin-binding protein (FGF-2), by using a competition experiment. Our results show that this method is an excellent platform for the fast screening of GAG-protein interactions. It requires very little sample (nmol/pmol per well) and can be useful for the determination of structure-activity relationships of synthetic GAG sequences, contributing to the understanding of the role of these polysaccharides in various biological processes.

\section{Experimental}

General procedures: Thin layer chromatography (TLC) analyses were performed on silica gel $60 \mathrm{~F}_{254}$ precoated on aluminium plates (Merck) and the compounds were detected by staining with sulfuric acid/ethanol (1:9), with cerium (IV) sulfate 
(10 g), phosphomolybdic acid (13 g), sulfuric acid $(60 \mathrm{~mL})$ solution in water $(1 \mathrm{~L})$ or with anisaldehyde solution (anisaldehyde $(25 \mathrm{~mL})$ with sulfuric acid $(25 \mathrm{~mL})$, ethanol $(450 \mathrm{~mL})$ and acetic acid $(1 \mathrm{~mL}))$ followed by heating at over $200^{\circ} \mathrm{C}$. Column chromatography was carried out on silica gel $60(0.2-0.5 \mathrm{~mm}, 0.2-0.063 \mathrm{~mm}$ or $0.040-$ $0.015 \mathrm{~mm}$; Merck). Optical rotations were determined with a Perkin-Elmer 341 polarimeter. ${ }^{1} \mathrm{H}$ - and ${ }^{13} \mathrm{C}-\mathrm{NMR}$ spectra were acquired on Bruker DPX-300, Avance III400 and DRX-500 spectrometers. Unit A refers to the reducing end monosaccharide in the NMR data. Electrospray mass spectra (ESI MS) were carried out with an Esquire 6000 ESI-Ion Trap from Bruker Daltonics. High resolution mass spectra (HR MS) were carried out by the Mass Spectrometry Service, CITIUS, Universidad de Sevilla. HR MS (electrospray) of compounds $\mathbf{3 0}$ and $\mathbf{1}$ were obtained with a Thermo LTQ Orbitrap Velos spectrometer at CCiT, Universitat de Barcelona. Microwave-based sulfation reactions were performed using a Biotage Initiator Eight synthesizer in sealed reaction vessels.

\section{Benzyl (4-methoxyphenyl 2-O-benzoyl-3-O-benzyl- $\beta$-D-glucopyranoside) uronate}

(6): TEMPO (2 mL of a $0.016 \mathrm{M}$ solution in $\left.\mathrm{CH}_{2} \mathrm{Cl}_{2}\right), \mathrm{Bu}_{4} \mathrm{NBr}(2 \mathrm{~mL}$ of a $0.08 \mathrm{M}$ solution in $\left.\mathrm{CH}_{2} \mathrm{Cl}_{2}\right)$ and $\mathrm{KBr}\left(0.65 \mathrm{~mL}\right.$ of a $0.5 \mathrm{M}$ solution in $\left.\mathrm{H}_{2} \mathrm{O}\right)$ were added dropwise at $0^{\circ} \mathrm{C}$ to a solution of diol $5(1.6 \mathrm{~g}, 3.33 \mathrm{mmol})$ in $\mathrm{CH}_{2} \mathrm{Cl}_{2}(33 \mathrm{~mL})$. A solution of $\mathrm{Ca}(\mathrm{ClO})_{2}(1.2 \mathrm{~g}, 8.3 \mathrm{mmol})$ and $\mathrm{NaHCO}_{3}(1.2 \mathrm{~g}, 14.3 \mathrm{mmol})$ in $\mathrm{H}_{2} \mathrm{O}(31$ $\mathrm{mL}$ ) was then added dropwise at $0^{\circ} \mathrm{C}$. After stirring for $1 \mathrm{~h}$ at $0^{\circ} \mathrm{C}$, the reaction was quenched by adding $\mathrm{Na}_{2} \mathrm{SO}_{3}\left(25 \mathrm{~mL}\right.$ of a $0.8 \mathrm{M}$ solution in $\mathrm{H}_{2} \mathrm{O}$ ). After stirring for 15 min at $0^{\circ} \mathrm{C}$, the reaction mixture was diluted with additional $\mathrm{CH}_{2} \mathrm{Cl}_{2}$ and $\mathrm{H}_{2} \mathrm{O}$, and the organic layer was then separated, washed with a solution of $\mathrm{Na}_{2} \mathrm{SO}_{3}(0.8 \mathrm{M})$ and brine, dried $\left(\mathrm{MgSO}_{4}\right)$, filtered, and concentrated. The residue was dissolved in DMF (45 mL) and benzyl bromide $(0.8 \mathrm{~mL}, 6.7 \mathrm{mmol})$ and $\mathrm{Bu}_{4} \mathrm{NI}(0.6 \mathrm{~g}, 1.7 \mathrm{mmol})$ were added. The 
mixture was stirred for $3 \mathrm{~h}$ at $60^{\circ} \mathrm{C}$, diluted with EtOAc, washed with $\mathrm{H}_{2} \mathrm{O}$, dried $\left(\mathrm{MgSO}_{4}\right)$, filtered, and concentrated. Flash chromatography (toluene/EtOAc, 13:1) gave $6(1.1 \mathrm{~g}, 56 \%)$. TLC $(6: 1$ toluene/EtOAc $) \mathrm{R}_{\mathrm{f}} 0.41 ;[\alpha]_{\mathrm{D}}^{20}-3.4^{\circ}\left(c 1.0, \mathrm{CH}_{2} \mathrm{Cl}_{2}\right) ;{ }^{1} \mathrm{H}-\mathrm{NMR}$ (300 MHz, $\left.\mathrm{CDCl}_{3}\right): \delta 7.94(\mathrm{~m}, 2 \mathrm{H}, \mathrm{Ar}), 7.50(\mathrm{~m}, 1 \mathrm{H}, \mathrm{Ar}), 7.36(\mathrm{~m}, 2 \mathrm{H}, \mathrm{Ar}), 7.28(\mathrm{~m}$, 5H, Ar), 7.09 (m, 5H, Ar), 6.83 (m, 2H, Ar), 6.62 (m, 2H, Ar), 5.42 (dd, 1H, H-2), 5.17 (2d, 2H, $\left.\mathrm{CH}_{2}(\mathrm{Bn})\right), 4.93$ (d, 1H, J 1,2 = 7.6 Hz, H-1), 4.70 (2d, 2H, $\left.\mathrm{CH}_{2}(\mathrm{Bn})\right), 4.12$ (dd, $\left.1 \mathrm{H}, J_{3,4}=J_{4,5}=9.5 \mathrm{~Hz}, \mathrm{H}-4\right), 3.96(\mathrm{~d}, 1 \mathrm{H}, \mathrm{H}-5), 3.71\left(\mathrm{dd}, 1 \mathrm{H}, J_{2,3}=9.2 \mathrm{~Hz}, \mathrm{H}-3\right), 3.63$ (s, 3H, Me(OMP)); ${ }^{13} \mathrm{C}-\mathrm{NMR}\left(75 \mathrm{MHz}, \mathrm{CDCl}_{3}\right): \delta 168.9,165.5(\mathrm{CO}(\mathrm{COOBn}, \mathrm{Bz}))$, 155.8, 151.3, 138.1, 135.3 (Ar-C), 133.5 (Ar-CH), 130.0, 129.8, 128.8, 128.7, 128.6, 128.4, 128.2, 127.8, (Ar-C, Ar-CH), 119.3, 114.6 (Ar-CH), 101.5 (C-1), 81.0 (C-3), 74.8, $74.7\left(\mathrm{CH}_{2}(\mathrm{Bn}), \mathrm{C}-5\right), 73.1(\mathrm{C}-2), 72.0(\mathrm{C}-4), 67.5\left(\mathrm{CH}_{2}(\mathrm{Bn})\right), 55.6(\mathrm{Me}(\mathrm{OMP}))$; HR MS: m/z: calcd for $\mathrm{C}_{34} \mathrm{H}_{32} \mathrm{O}_{9} \mathrm{Na}$ : 607.1944; found: $607.1946[M+\mathrm{Na}]^{+}$.

\section{Benzyl (4-methoxyphenyl 2-O-benzoyl-3- $O$-benzyl-4- $O$-levulinoyl- $\beta$-D-}

glucopyranoside) uronate (7): Compound 6 (2.1 g, $3.6 \mathrm{mmol}), 1,3-$

dicyclohexylcarbodiimide (1.11 g, $5.39 \mathrm{mmol})$, DMAP (44 mg, $0.36 \mathrm{mmol})$ and levulinic acid $(1.83 \mathrm{~mL}, 18.0 \mathrm{mmol})$ were dissolved in $\mathrm{CH}_{2} \mathrm{Cl}_{2}(25 \mathrm{~mL})$. After stirring for $3 \mathrm{~h}$, the mixture was diluted with $\mathrm{CH}_{2} \mathrm{Cl}_{2}$ and washed with saturated aqueous $\mathrm{NaHCO}_{3}$. The organic phase was dried with $\mathrm{MgSO}_{4}$, filtered, and concentrated in vacuo. The residue was purified by flash cromatography on silica gel (2:1 hexane/EtOAc) to give 7 as a white solid (1.98 g, 81\%). TLC (2:1 hexane/EtOAc) $\mathrm{R}_{\mathrm{f}}$ 0.28; $[\alpha]_{\mathrm{D}}^{20}+8.4^{\mathrm{o}}\left(c 1.0, \mathrm{CH}_{2} \mathrm{Cl}_{2}\right) ;{ }^{1} \mathrm{H}-\mathrm{NMR}\left(300 \mathrm{MHz}, \mathrm{CDCl}_{3}\right): \delta 7.94(\mathrm{~m}, 2 \mathrm{H}, \mathrm{Ar})$, $7.52(\mathrm{~m}, 1 \mathrm{H}, \mathrm{Ar}), 7.38(\mathrm{~m}, 2 \mathrm{H}, \mathrm{Ar}), 7.28(\mathrm{~m}, 5 \mathrm{H}, \mathrm{Ar}), 7.09$ (m, 5H, Ar), $6.83(\mathrm{~m}, 2 \mathrm{H}$, Ar), $6.65(\mathrm{~m}, 2 \mathrm{H}, \mathrm{Ar}), 5.49\left(\mathrm{dd}, 1 \mathrm{H}, J_{1,2}=7.2 \mathrm{~Hz}, J_{2,3}=8.9 \mathrm{~Hz}, \mathrm{H}-2\right), 5.40\left(\mathrm{dd}, 1 \mathrm{H}, J_{3,4}\right.$ $\left.=9.0 \mathrm{~Hz}, J_{4,5}=9.6 \mathrm{~Hz}, \mathrm{H}-4\right), 5.08\left(\mathrm{~s}, 2 \mathrm{H}, \mathrm{CH}_{2}(\mathrm{Bn})\right), 4.99(\mathrm{~d}, 1 \mathrm{H}, \mathrm{H}-1), 4.58(2 \mathrm{~d}, 2 \mathrm{H}$, $\left.\mathrm{CH}_{2}(\mathrm{Bn})\right), 4.08(\mathrm{~d}, 1 \mathrm{H}, \mathrm{H}-5), 3.88\left(\mathrm{dd}, 1 \mathrm{H}, J_{2,3}=J_{3,4}=8.9 \mathrm{~Hz}, \mathrm{H}-3\right), 3.66(\mathrm{~s}, 3 \mathrm{H}$, 
$\mathrm{Me}(\mathrm{OMP})$ ), 2.60-2.17 (m, 4H, $\left.\mathrm{CH}_{2}(\mathrm{Lev})\right), 2.05$ (s, 3H, $\left.\mathrm{CH}_{3}(\mathrm{Lev})\right) ;{ }^{13} \mathrm{C}-\mathrm{NMR}(75 \mathrm{MHz}$, $\left.\mathrm{CDCl}_{3}\right): \delta 205.9(\mathrm{CO}(\mathrm{Lev})), 171.2,166.9,165.0$ (CO (COOBn, Bz, Lev)), 155.9, 151.1, 137.4, 135.1 (Ar-C), 133.4 (Ar-CH), 129.8, 129.5, 128.6, 128.5, 128.3, 128.0, 127.7, (Ar-C, Ar-CH), 119.3, 114.5 (Ar-CH), 101.0 (C-1), 78.9 (C-3), $73.9\left(\mathrm{CH}_{2}(\mathrm{Bn})\right), 72.9$ (C-5), 72.8 (C-2), $71.0(\mathrm{C}-4), 67.7\left(\mathrm{CH}_{2}(\mathrm{Bn})\right), 55.6(\mathrm{Me}(\mathrm{OMP})), 37.6\left(\mathrm{CH}_{2}(\mathrm{Lev})\right), 29.7$ $\left(\mathrm{CH}_{3}(\mathrm{Lev})\right), 27.7\left(\mathrm{CH}_{2}(\mathrm{Lev})\right)$; HR MS: m/z: calcd for $\mathrm{C}_{39} \mathrm{H}_{38} \mathrm{O}_{11} \mathrm{Na}$ : 705.2312; found: $705.2316[M+\mathrm{Na}]^{+}$.

\section{Benzyl 2-O-benzoyl-3- $O$-benzyl-4- $O$-levulinoyl- $\alpha, \beta$-D-glucopyranosuronate (8):}

CAN (8.1 g, $14 \mathrm{mmol})$ was added to a solution of the glycoside 7 (1.9 g, $2.8 \mathrm{mmol})$ in toluene-acetonitrile-water $(1: 6: 1,75 \mathrm{~mL})$ and the mixture was stirred for $1.5 \mathrm{~h}$ at r.t. The mixture was then diluted with EtOAc, washed with $\mathrm{H}_{2} \mathrm{O}$ and $\mathrm{NaHCO}_{3}$, dried $\left(\mathrm{MgSO}_{4}\right)$, filtered and concentrated under reduced pressure. Flash chromatography on silica gel (toluene/EtOAc 1:0 $\rightarrow$ 3:1) afforded the corresponding hemiacetal 8 (960 mg, $60 \%)$ as a mixture of $\alpha / \beta$ anomers (9:1). TLC (2:1 toluene/EtOAc) $\mathrm{R}_{\mathrm{f}} 0.47 ;{ }^{1} \mathrm{H}-\mathrm{NMR}$ (300 MHz, $\mathrm{CDCl}_{3}$ ) (for $\alpha$ anomer): $\delta 8.03(\mathrm{~m}, 2 \mathrm{H}, \mathrm{Ar}), 7.58(\mathrm{~m}, 1 \mathrm{H}, \mathrm{Ar}), 7.43(\mathrm{~m}, 2 \mathrm{H}$, Ar), $7.34(\mathrm{~m}, 5 \mathrm{H}, \mathrm{Ar}), 7.21(\mathrm{~m}, 5 \mathrm{H}, \mathrm{Ar}), 5.68\left(\mathrm{~d}, 1 \mathrm{H}, J_{1,2}=3.4 \mathrm{~Hz}, \mathrm{H}-1\right), 5.29(\mathrm{dd}, 1 \mathrm{H}$, $\left.J_{3,4}=J_{4,5}=8.9 \mathrm{~Hz}, \mathrm{H}-4\right), 5.16(\mathrm{dd}, 1 \mathrm{H}, \mathrm{H}-2), 5.06\left(2 \mathrm{~d}, 2 \mathrm{H}, \mathrm{CH}_{2}(\mathrm{Bn})\right), 4.70(2 \mathrm{~d}, 2 \mathrm{H}$, $\left.\mathrm{CH}_{2}(\mathrm{Bn})\right), 4.61\left(\mathrm{~d}, 1 \mathrm{H}, J_{4,5}=8.9 \mathrm{~Hz}, \mathrm{H}-5\right), 4.23\left(\mathrm{dd}, 1 \mathrm{H}, J_{2,3}=J_{3,4}=8.9 \mathrm{~Hz}, \mathrm{H}-3\right)$, 2.58-2.21 (m, 4H, $\mathrm{CH}_{2}(\mathrm{Lev})$ ), 2.09 (s, 3H, $\mathrm{CH}_{3}(\mathrm{Lev})$ ); ${ }^{13} \mathrm{C}-\mathrm{NMR}\left(75 \mathrm{MHz}, \mathrm{CDCl}_{3}\right.$ ) (for $\alpha$ anomer): $\delta 206.4$ (CO(Lev)), 171.4, 168.5, 165.7 (CO (COOBn, Bz, Lev)), 137.9127.7 (Ar), 90.3 (C-1), 76.1(C-3), $74.8\left(\mathrm{CH}_{2}(\mathrm{Bn})\right), 72.9$ (C-2), 71.0 (C-4), 68.9 (C-5), $68.0\left(\mathrm{CH}_{2}(\mathrm{Bn})\right), 37.6\left(\mathrm{CH}_{2}(\mathrm{Lev})\right), 29.7\left(\mathrm{CH}_{3}(\mathrm{Lev})\right), 27.7\left(\mathrm{CH}_{2}(\mathrm{Lev})\right) ;{ }^{1} \mathrm{H}-\mathrm{NMR}(300$ $\mathrm{MHz}, \mathrm{CDCl}_{3}$ ) (selected data for $\beta$ anomer): $\delta 7.99(\mathrm{~m}, 2 \mathrm{H}, \mathrm{Ar}), 7.58$ (m, 1H, Ar), 7.43 $(\mathrm{m}, 2 \mathrm{H}, \mathrm{Ar}), 7.26(\mathrm{~m}, 5 \mathrm{H}, \mathrm{Ar}), 7.18(\mathrm{~m}, 5 \mathrm{H}, \mathrm{Ar}), 5.37\left(\mathrm{dd}, 1 \mathrm{H}, J_{3,4}=J_{4,5}=9.0 \mathrm{~Hz}, \mathrm{H}-\right.$ 4), $5.20(\mathrm{dd}, 1 \mathrm{H}, \mathrm{H}-2), 4.86\left(\mathrm{~d}, 1 \mathrm{H}, J_{1,2}=6.9 \mathrm{~Hz}, \mathrm{H}-1\right), 4.15\left(\mathrm{~d}, 1 \mathrm{H}, J_{4,5}=9.2 \mathrm{~Hz}, \mathrm{H}-5\right)$, 
$3.95\left(\mathrm{dd}, 1 \mathrm{H}, J_{2,3}=J_{3,4}=8.3 \mathrm{~Hz}, \mathrm{H}-3\right), 2.58-2.21\left(\mathrm{~m}, 4 \mathrm{H}, \mathrm{CH}_{2}(\mathrm{Lev})\right), 2.09(\mathrm{~s}, 3 \mathrm{H}$, $\left.\mathrm{CH}_{3}(\mathrm{Lev})\right)$; ${ }^{13} \mathrm{C}-\mathrm{NMR}\left(75 \mathrm{MHz}, \mathrm{CDCl}_{3}\right.$ ) (selected data for $\beta$ anomer from HMQC experiment): $\delta 96.3$ (C-1), 77.6 (C-3), 72.7 (C-5), 70.8 (C-4); HR MS: $m / z$ : calcd for $\mathrm{C}_{32} \mathrm{H}_{32} \mathrm{O}_{10} \mathrm{Na}$ : 599.1893; found: $599.1911[M+\mathrm{Na}]^{+}$.

$O$-(Benzyl 2- $O$-benzoyl-3- $O$-benzyl-4- $O$-levulinoyl- $\alpha, \beta$-D-glucopyranosyluronate) trichloroacetimidate (2): Trichloroacetonitrile $(0.83 \mathrm{~mL}, 8.3 \mathrm{mmol})$ and $\mathrm{K}_{2} \mathrm{CO}_{3}(126$ $\mathrm{mg}, 0.91 \mathrm{mmol})$ were added to $\mathbf{8}(480 \mathrm{mg}, 0.83 \mathrm{mmol})$ in dry $\mathrm{CH}_{2} \mathrm{Cl}_{2}(4.8 \mathrm{~mL})$. After stirring at room temperature for $4 \mathrm{~h}$, the mixture was filtered off and concentrated in vacuo to obtain $2(574 \mathrm{mg}, 96 \%)$. TLC (2:1 toluene/EtOAc) $\mathrm{R}_{\mathrm{f}} 0.55 ;{ }^{1} \mathrm{H}-\mathrm{NMR}(300$ $\left.\mathrm{MHz}, \mathrm{CDCl}_{3}\right)$ : (for $\alpha$ anomer): $\delta 8.59(\mathrm{~s}, 1 \mathrm{H}, \mathrm{NH}), 7.95(\mathrm{~m}, 2 \mathrm{H}, \mathrm{Ar}), 7.58(\mathrm{~m}, 1 \mathrm{H}, \mathrm{Ar})$, $7.43(\mathrm{~m}, 2 \mathrm{H}, \mathrm{Ar}), 7.37$ (m, 5H, Ar), 7.20 (m, 5H, Ar), 6.74 (d, 1H, $\left.J_{1,2}=3.3 \mathrm{~Hz}, \mathrm{H}-1\right)$, 5.44 (dd, 1H, H-2), 5.38 (dd, 1H, H-4), 5.15 (2d, 2H, $\left.\mathrm{CH}_{2}(\mathrm{Bn})\right), 4.72(2 \mathrm{~d}, 2 \mathrm{H}$, $\left.\mathrm{CH}_{2}(\mathrm{Bn})\right), 4.52\left(\mathrm{~d}, 1 \mathrm{H}, J_{4,5}=10.1 \mathrm{~Hz}, \mathrm{H}-5\right), 4.29\left(\mathrm{dd}, 1 \mathrm{H}, J_{2,3}=J_{3,4}=9.7 \mathrm{~Hz}, \mathrm{H}-3\right)$, 2.66-2.20 (m, 4H, $\left.\mathrm{CH}_{2}(\mathrm{Lev})\right), 2.12\left(\mathrm{~s}, 3 \mathrm{H}, \mathrm{CH}_{3}(\mathrm{Lev})\right) ;{ }^{13} \mathrm{C}-\mathrm{NMR}\left(75 \mathrm{MHz}, \mathrm{CDCl}_{3}\right)$ (for $\alpha$ anomer): $\delta 206.4$ (CO(Lev)), 171.4, 168.5, 165.7 (CO(COOBn, Bz, Lev)), 160.0 $(\mathrm{C}=\mathrm{NH})$, 137.4-127.7 (Ar), $93.1(\mathrm{C}-1), 90.8\left(\mathrm{CCl}_{3}\right), 75.7(\mathrm{C}-3), 74.9\left(\mathrm{CH}_{2}(\mathrm{Bn})\right)$, 71.3, 71.2, $70.9\left(\mathrm{C}-2\right.$, C-5, C-4), $67.9\left(\mathrm{CH}_{2}(\mathrm{Bn})\right), 37.6\left(\mathrm{CH}_{2}(\mathrm{Lev})\right), 29.7\left(\mathrm{CH}_{3}(\mathrm{Lev})\right), 27.7$ $\left(\mathrm{CH}_{2}(\mathrm{Lev})\right)$; HR MS: $\mathrm{m} / z$ : calcd for $\mathrm{C}_{34} \mathrm{H}_{32} \mathrm{Cl}_{3} \mathrm{NO}_{10} \mathrm{Na}$ : 742.0989; found: 742.0984 $[M+\mathrm{Na}]^{+}$.

\section{Methyl (dimethylthexylsilyl 3-O-benzyl-2,4-di- $O$-levulinoyl- $\beta$-L-idopyranoside)}

uronate (10): $\mathrm{Lev}_{2} \mathrm{O}$ preparation: $\mathrm{LevOH}(4.7 \mathrm{~mL}, 45.4 \mathrm{mmol})$ was added at $0^{\circ} \mathrm{C}$ to a solution of 1,3-dicyclohexylcarbodiimide (4.68 g, $22.7 \mathrm{mmol})$ in $\mathrm{CH}_{2} \mathrm{Cl}_{2}(38 \mathrm{~mL})$. After stirring for $5 \mathrm{~min}$ at room temperature, the mixture was cooled and filtered to give a solution of $\mathrm{Lev}_{2} \mathrm{O}$ in $\mathrm{CH}_{2} \mathrm{Cl}_{2}$. 
$\mathrm{Lev}_{2} \mathrm{O}\left(15.0 \mathrm{~mL}\right.$ of a $0.76 \mathrm{M}$ solution in $\left.\mathrm{CH}_{2} \mathrm{Cl}_{2}\right)$ was added at room temperature to a mixture of 9 (1.0 g, $2.3 \mathrm{mmol})$ and DMAP (41 mg, $0.34 \mathrm{mmol})$ in dry Py (40 mL). The mixture was stirred for $22 \mathrm{~h}$, diluted with $\mathrm{CH}_{2} \mathrm{Cl}_{2}$, and washed with $1 \mathrm{M} \mathrm{HCl}$, saturated aqueous $\mathrm{NaHCO}_{3}$, and $\mathrm{H}_{2} \mathrm{O}$. The organic phase was dried $\left(\mathrm{MgSO}_{4}\right)$, filtered and concentrated to dryness. The residue was purified by column chromatography (hexane/EtOAc 2:1) to afford $\mathbf{1 0}(1.55 \mathrm{~g}, 98 \%)$. TLC (hexane/EtOAc $1: 1) \mathrm{R}_{\mathrm{f}} 0.26 ;[\alpha]_{\mathrm{D}}^{20}$ $+19^{\circ}\left(c\right.$ 1.0, $\left.\mathrm{CHCl}_{3}\right) ;{ }^{1} \mathrm{H}-\mathrm{NMR}\left(300 \mathrm{MHz}, \mathrm{CDCl}_{3}\right): \delta 7.34(\mathrm{~m}, 5 \mathrm{H}, \mathrm{Ar}), 5.16(\mathrm{~m}, 1 \mathrm{H}, \mathrm{H}-$ 4), $5.08\left(\mathrm{~d}, 1 \mathrm{H}, J_{1,2}=1.6 \mathrm{~Hz}, \mathrm{H}-1\right), 4.93(\mathrm{~m}, 1 \mathrm{H}, \mathrm{H}-2), 4.76,4.69\left(2 \mathrm{~d}, 2 \mathrm{H}, \mathrm{CH}_{2}(\mathrm{Bn})\right)$, $4.59\left(\mathrm{~d}, 1 \mathrm{H}, J_{4,5}=2.1 \mathrm{~Hz}, \mathrm{H}-5\right), 3.86\left(\mathrm{t}, 1 \mathrm{H}, J_{2,3}=J_{3,4}=2.7 \mathrm{~Hz}, \mathrm{H}-3\right), 3.78(\mathrm{~s}, 3 \mathrm{H}$, COOMe), 2.92-2.50 (m, 8H, $\left.\mathrm{CH}_{2}(\mathrm{Lev})\right), 2.18$ (s, 6H, $\left.\mathrm{CH}_{3}(\mathrm{Lev})\right), 1.61$ (hp, 1H, $\left.\mathrm{CH}\left(\mathrm{CH}_{3}\right)_{2}\right), 0.87-0.83\left(12 \mathrm{H}, \mathrm{CH}\left(\mathrm{CH}_{3}\right)_{2}, \mathrm{C}\left(\mathrm{CH}_{3}\right)_{2}\right), 0.23,0.14$ (2s, 6H, $\left.\mathrm{Si}\left(\mathrm{CH}_{3}\right)_{2}\right)$; ${ }^{13} \mathrm{C}-\mathrm{NMR}\left(75 \mathrm{MHz}, \mathrm{CDCl}_{3}\right): \delta$ 206.5, 206.3 (CO(Lev)), 172.3, 172.1 (CO(Lev)), 167.9 (COOMe), 137.3 (Ar-C), 128.6, 128.2, 127.8 (Ar-CH), 93.1 (C-1), 74.4 (C-3), 73.1 $\left(\mathrm{CH}_{2}(\mathrm{Bn})\right), 72.7$ (C-5), 68.0 (C-2), 67.2 (C-4), 52.4 (COOMe), 38.0, $37.8\left(\mathrm{CH}_{2}(\mathrm{Lev})\right)$, $34.1\left(\mathrm{CH}\left(\mathrm{CH}_{3}\right)_{2}\right), 30.0,29.9\left(\mathrm{CH}_{3}(\mathrm{Lev})\right), 28.1\left(\mathrm{CH}_{2}(\mathrm{Lev})\right), 25.0\left(\mathrm{C}\left(\mathrm{CH}_{3}\right)_{2}\right), 20.3,20.0$, 18.7, $18.5\left(\mathrm{CH}\left(\mathrm{CH}_{3}\right)_{2}, \mathrm{C}\left(\mathrm{CH}_{3}\right)_{2}\right),-1.8,-3.5\left(\mathrm{Si}\left(\mathrm{CH}_{3}\right)_{2}\right)$; HR MS: $m / z$ : calcd for $\mathrm{C}_{32} \mathrm{H}_{48} \mathrm{O}_{11} \mathrm{SiNa}$ : 659.2864; found: $659.2847[M+\mathrm{Na}]^{+}$.

Methyl 3- $O$-benzyl-2,4-di- $O$-levulinoyl- $\alpha, \beta$-L-idopyranosuronate (11): An excess of $(\mathrm{HF})_{\mathrm{n}} \cdot \mathrm{Py}(7.2 \mathrm{~mL})$ was added at $-10^{\circ} \mathrm{C}$ under an argon atmosphere to a solution of $\mathbf{1 0}$ $(1.63 \mathrm{~g}, 2.56 \mathrm{mmol})$ in dry THF $(37 \mathrm{~mL})$. After $19 \mathrm{~h}$ at $0^{\circ} \mathrm{C}$ the mixture was diluted with $\mathrm{CH}_{2} \mathrm{Cl}_{2}$ and washed with $\mathrm{H}_{2} \mathrm{O}$ and saturated $\mathrm{NaHCO}_{3}$ solution until neutral $\mathrm{pH}$. The organic layers were dried $\left(\mathrm{MgSO}_{4}\right)$, filtered and concentrated in vacuo. The residue was purified by column chromatography (toluene/EtOAc 1:3) to afford $\mathbf{1 1}$ as a mixture of $\alpha / \beta$ anomers (1:1) (970 mg, 77\%). TLC (hexane/EtOAc 1:2) $\mathrm{R}_{\mathrm{f}} 0.13 ;{ }^{1} \mathrm{H}-\mathrm{NMR}(300$ $\left.\mathrm{MHz}, \mathrm{CDCl}_{3}\right): \delta 7.34(\mathrm{~m}, 10 \mathrm{H}, \mathrm{Ar}, \mathrm{Ar}$ '), 5.30 (m, 1H, H-1), 5.26 (m, 1H, H-4), 5.16 (m, 
1H, H-4'), 5.14 (bs, 1H, H-1'), 4.98 (d, 1H, J4,5 = 2.3 Hz, H-5), 4.95 (m, 1H, H-2'), 4.85 (m, 1H, H-2), 4.78, 4.74 (2m, 4H, $\left.\mathrm{CH}_{2}(\mathrm{Bn}), \mathrm{CH}_{2}(\mathrm{Bn})^{\prime}\right), 4.69$ (d, 1H, J4,5 $=2.0 \mathrm{~Hz}, \mathrm{H}-$ 5'), 4.18 (bd, 1H, OH), 3.96 (bt, 2H, H-3, H-3'), 3.81, 3.80 (2s, 6H, COOMe, COOMe'), 2.91-2.45 (m, 16H, $\mathrm{CH}_{2}(\mathrm{Lev}), \mathrm{CH}_{2}(\mathrm{Lev})$ '), 2.19-2-17 (3s, 12H, $\mathrm{CH}_{3}(\mathrm{Lev})$, $\left.\mathrm{CH}_{3}(\mathrm{Lev}) '\right) ;{ }^{13} \mathrm{C}-\mathrm{NMR}\left(75 \mathrm{MHz}, \mathrm{CDCl}_{3}\right.$ ): $\delta 207.3,206.5,206.3$ (CO(Lev), CO(Lev)'), 172.4, 171.9, 171.8 (CO(Lev), CO(Lev)'), 168.8, 168.1 (COOMe, COOMe'), 137.0, 136.6 (Ar-C), 129.1, 128.7, 128.6, 128.4, 128.3, 128.2, 128.0, 127.8 (Ar-C, Ar-CH), 93.0 (C-1), 92.1 (C-1'), 73.5, $\left.73.4\left(\mathrm{CH}_{2}(\mathrm{Bn}), \mathrm{CH}_{2}(\mathrm{Bn})\right)^{\prime}\right), 73.1,72.7,72.3$ (C-3, C-3', C-5’), 68.0 (C-2'), 67.1 (C-4, C-4'), 66.7 (C-2), 65.6 (C-5), 52.6 (COOMe, COOMe'), 38.1, 37.8, 37.7 ( $\mathrm{CH}_{2}(\mathrm{Lev}), \mathrm{CH}_{2}(\mathrm{Lev})$ '), $29.8\left(\mathrm{CH}_{3}(\mathrm{Lev}), \mathrm{CH}_{3}(\mathrm{Lev})\right.$ ') $, 28.0,27.9,27.8$ $\left(\mathrm{CH}_{2}(\mathrm{Lev}), \mathrm{CH}_{2}(\mathrm{Lev})^{\prime}\right)$; HR MS: $m / z$ : calcd for $\mathrm{C}_{24} \mathrm{H}_{30} \mathrm{O}_{11} \mathrm{Na}$ : 517.1686; found: $517.1666[M+\mathrm{Na}]^{+}$.

\section{$O$-(Methyl}

\section{3-O-benzyl-2,4-di- $O$-levulinoyl- $\alpha, \beta$-L-idopyranosyluronate)}

trichloroacetimidate (3): $\mathrm{K}_{2} \mathrm{CO}_{3}(207 \mathrm{mg}, 1.67 \mathrm{mmol})$ and trichloroacetonitrile $(3.0$ $\mathrm{mL}, 30 \mathrm{mmol})$ were added at room temperature to a solution of $\mathbf{1 1}(750 \mathrm{mg}, 1.52 \mathrm{mmol})$ in dry $\mathrm{CH}_{2} \mathrm{Cl}_{2}(15 \mathrm{~mL})$. After stirring for $11 \mathrm{~h}$ at room temperature, the reaction mixture was filtered through a pad of Celite and concentrated to dryness to obtain $\mathbf{3}$ as a mixture of $\alpha / \beta$ anomers $\left(920 \mathrm{mg}, 95 \%\right.$ ). TLC (toluene/EtOAc 1:4) $\mathrm{R}_{\mathrm{f}} 0.44,0.58$ ( $\alpha$ and $\beta$ anomers); ${ }^{1} \mathrm{H}-\mathrm{NMR}\left(300 \mathrm{MHz}, \mathrm{CDCl}_{3}\right.$ ) (data for major anomer): $\delta 8.68(\mathrm{~s}, 1 \mathrm{H}, \mathrm{NH}$ ), 7.34 (m, 5H, Ar), 6.23 (d, 1H, $\left.J_{1,2}=1.8 \mathrm{~Hz}, \mathrm{H}-1\right), 5.27$ (m, 1H, H-2), 5.22 (m, 1H, H-4), $4.80\left(\mathrm{~d}, 1 \mathrm{H}, J_{4,5}=2.0 \mathrm{~Hz}, \mathrm{H}-5\right), 4.80-4.68\left(\mathrm{~m}, 2 \mathrm{H}, \mathrm{CH}_{2}(\mathrm{Bn})\right), 3.99\left(\mathrm{t}, 1 \mathrm{H}, J_{2,3}=J_{3,4}=3.0\right.$ Hz, H-3), 3.78 (s, 3H, COOMe), 2.90-2.49 (m, 8H, $\mathrm{CH}_{2}(\mathrm{Lev})$ ), 2.16 (s, 6H, $\mathrm{CH}_{3}(\mathrm{Lev})$ ); ${ }^{13} \mathrm{C}-\mathrm{NMR}\left(75 \mathrm{MHz}, \mathrm{CDCl}_{3}\right.$ ) (data for major anomer): $\delta$ 206.4, $206.3(\mathrm{CO}(\mathrm{Lev})), 172.1$, $172.0(\mathrm{CO}(\mathrm{Lev})), 167.2(\mathrm{COOMe}), 160.4(\mathrm{C}=\mathrm{NH}), 136.9$ (Ar-C), 128.6, 128.3, 127.9 (Ar-CH), $94.4(\mathrm{C}-1), 90.8\left(\mathrm{CCl}_{3}\right), 73.6(\mathrm{C}-3), 73.4\left(\mathrm{CH}_{2}(\mathrm{Bn})\right), 73.2(\mathrm{C}-5), 67.3(\mathrm{C}-4)$, 
65.8 (C-2), 52.7 (COOMe), 37.8, $37.7\left(\mathrm{CH}_{2}(\mathrm{Lev})\right)$, 29.9, $29.8\left(\mathrm{CH}_{3}(\mathrm{Lev})\right), 28.0,27.9$ $\left(\mathrm{CH}_{2}(\mathrm{Lev})\right) ;{ }^{1} \mathrm{H}-\mathrm{NMR}\left(300 \mathrm{MHz}, \mathrm{CDCl}_{3}\right.$ ) (data for minor anomer): $\delta 8.69(\mathrm{~s}, 1 \mathrm{H}, \mathrm{NH})$, 7.34 (m, 5H, Ar), 6.40 (bs, 1H, H-1), 5.27 (m, 1H, H-4), 5.11 (m, 1H, H-2), 5.04 (d, 1H, $\left.J_{4,5}=1.8 \mathrm{~Hz}, \mathrm{H}-5\right), 4.80-4.68\left(\mathrm{~m}, 2 \mathrm{H}, \mathrm{CH}_{2}(\mathrm{Bn})\right), 3.87(\mathrm{~m}, 1 \mathrm{H}, \mathrm{H}-3), 3.78(\mathrm{~s}, 3 \mathrm{H}$, COOMe), 2.90-2.49 (m, 8H, $\left.\mathrm{CH}_{2}(\mathrm{Lev})\right), 2.17$ (s, 6H, $\left.\mathrm{CH}_{3}(\mathrm{Lev})\right) ;{ }^{13} \mathrm{C}-\mathrm{NMR}(75 \mathrm{MHz}$, $\mathrm{CDCl}_{3}$ ) (data for minor anomer): $\delta$ 206.4, $206.3(\mathrm{CO}(\mathrm{Lev})), 171.8,171.7(\mathrm{CO}(\mathrm{Lev}))$, 168.1 (COOMe), $160.1(\mathrm{C}=\mathrm{NH}), 137.2$ (Ar-C), 128.4, 127.9, 127.7 (Ar-CH), 95.0 (C-1), $90.5\left(\mathrm{CCl}_{3}\right), 72.6\left(\mathrm{CH}_{2}(\mathrm{Bn})\right), 71.5(\mathrm{C}-3), 67.8(\mathrm{C}-5), 67.3(\mathrm{C}-4), 65.2(\mathrm{C}-2), 52.7$ (COOMe), 37.9, $37.8\left(\mathrm{CH}_{2}(\mathrm{Lev})\right), 29.9,29.8\left(\mathrm{CH}_{3}(\mathrm{Lev})\right)$, 28.0, $27.9\left(\mathrm{CH}_{2}(\mathrm{Lev})\right)$; HR MS: $m / z$ : calcd for $\mathrm{C}_{26} \mathrm{H}_{30} \mathrm{Cl}_{3} \mathrm{NO}_{11} \mathrm{Na}$ : 660.0782; found: 660.0782 [M+Na] $]^{+}$

\section{1,3,4,6-Tetra- $O$-acetyl-2-deoxy-2-trifluoroacetamido- $\alpha, \beta$-D-galactopyranose $(12)^{61}$,}

62: Galactosamine hydrochloride (21 g, $93.4 \mathrm{mmol})$ was suspended in $\mathrm{MeOH}$ (250 mL). $\mathrm{NaOMe}(105 \mathrm{~mL}, 1.3 \mathrm{M}$ solution in $\mathrm{MeOH})$ was added at room temperature. After stirring for $30 \mathrm{~min}$, TFA anhydride $(14.2 \mathrm{~mL}, 98.1 \mathrm{mmol})$ was added at $0^{\circ} \mathrm{C}$. After stirring for $10 \mathrm{~min}, \mathrm{Et}_{3} \mathrm{~N}(13.6 \mathrm{~mL}, 94.0 \mathrm{mmol})$ was added. After stirring for $28 \mathrm{~h}$ at room temperature, the reaction mixture was concentrated in vacuo. The residue was dissolved in Py (420 ml) and DMAP (6.4 g, $46.7 \mathrm{mmol})$ and acetic anhydride (105.8 $\mathrm{mL}, 1.1 \mathrm{~mol}$ ) were added at $0{ }^{\circ} \mathrm{C}$. The reaction was stirred for $72 \mathrm{~h}$ at room temperature, diluted with EtOAc, washed with $\mathrm{H}_{2} \mathrm{O}, 1 \mathrm{M} \mathrm{HCl}$ and $\mathrm{NaHCO}_{3}$, dried $\left(\mathrm{MgSO}_{4}\right)$, filtered, and concentrated. Flash chromatography (hexane/EtOAc, 3:1) gave 12 (28.4 g, 66\%) as a mixture of $\alpha / \beta$ anomers (1:0.8). TLC (2:1 hexane/EtOAc) $\mathrm{R}_{\mathrm{f}} 0.27 ;{ }^{1} \mathrm{H}-\mathrm{NMR}(300$ $\mathrm{MHz}, \mathrm{CDCl}_{3}$ ) (data for $\alpha$ anomer): $\delta 6.55(\mathrm{bs}, 1 \mathrm{H}, \mathrm{NH}), 6.28\left(\mathrm{~d}, 1 \mathrm{H}, J_{1,2}=3.6 \mathrm{~Hz}, \mathrm{H}-\right.$ 1), $5.46(\mathrm{dd}, 1 \mathrm{H}, \mathrm{H}-4), 5.29$ (dd, $\left.1 \mathrm{H}, J_{2,3}=11.4 \mathrm{~Hz}, J_{3,4}=3.5 \mathrm{~Hz}, \mathrm{H}-3\right), 4.68(\mathrm{~m}, 1 \mathrm{H}$, H-2), 4.28-4.03 (m, 3H, H-5, H-6a, H-6b), 2.18-2.00 (m, 12H, $\left.\mathrm{CH}_{3}\right) ;{ }^{1} \mathrm{H}-\mathrm{NMR}(300$ $\mathrm{MHz}, \mathrm{CDCl}_{3}$ ) (data for $\beta$ anomer): $\delta 7.02(\mathrm{bs}, 1 \mathrm{H}, \mathrm{NH}), 5.77\left(\mathrm{~d}, 1 \mathrm{H}, J_{1,2}=8.7 \mathrm{~Hz}, \mathrm{H}-\right.$ 
1), 5.40 (dd, 1H, H-4), 5.18 (dd, $\left.1 \mathrm{H}, J_{2,3}=11.3 \mathrm{~Hz}, J_{3,4}=3.3 \mathrm{~Hz}, \mathrm{H}-3\right), 4.49(\mathrm{~m}, 1 \mathrm{H}$, H-2), 4.28-4.03 (m, 3H, H-5, H-6a, H-6b), 2.18-2.00 (m, 12H, $\mathrm{CH}_{3}$ ); ESI MS: $m / z$ : calcd for $\mathrm{C}_{16} \mathrm{H}_{20} \mathrm{~F}_{3} \mathrm{NO}_{10} \mathrm{Na}$ : 466.1; found: $466.1[M+\mathrm{Na}]^{+}$.

4-Methoxyphenyl 3,4,6-tri- $O$-acetyl-2-deoxy-2-trifluoroacetamido- $\beta$-Dgalactopyranoside (13): TMSOTf $(320 \mu \mathrm{L}, 1.7 \mathrm{mmol})$ was added to a cooled $\left(0^{\circ} \mathrm{C}\right)$ solution of 12 (5.1 g, $11.5 \mathrm{mmol})$ and 4-methoxyphenol (2.6 g, $20.7 \mathrm{mmol})$ in dry $\mathrm{CH}_{2} \mathrm{Cl}_{2}(51 \mathrm{~mL})$. The mixture was stirred for $1 \mathrm{~h}$ at $0^{\circ} \mathrm{C}$ and TEA $(1 \mathrm{~mL})$ was then added. The mixture was diluted with EtOAc and washed with $\mathrm{H}_{2} \mathrm{O}$, saturated aqueous $\mathrm{NaHCO}_{3}$ and $\mathrm{H}_{2} \mathrm{O}$. The organic phase was dried $\left(\mathrm{MgSO}_{4}\right)$, filtered, and concentrated. The residue was purified by silica gel chromatography (toluene/EtOAc $4: 1 \rightarrow 1: 1$ ) to give $13(2.4 \mathrm{~g}, 42 \%$ ) and starting material ( $\alpha$ anomer, $2.4 \mathrm{~g}, 40 \%)$. TLC (4:1

toluene/EtOAc) $\mathrm{R}_{\mathrm{f}} 0.17 ;[\alpha]_{\mathrm{D}}^{20}-3.5^{\circ}\left(c 1.0, \mathrm{CHCl}_{3}\right) ;{ }^{1} \mathrm{H}-\mathrm{NMR}\left(300 \mathrm{MHz}, \mathrm{CDCl}_{3}\right): \delta$ $6.98\left(\mathrm{~d}, 1 \mathrm{H}, J_{2, \mathrm{NH}}=9.1 \mathrm{~Hz}, \mathrm{NH}\right), 6.92(\mathrm{~m}, 2 \mathrm{H}, \mathrm{Ar}), 6.78(\mathrm{~m}, 2 \mathrm{H}, \mathrm{Ar}), 5.41\left(\mathrm{~d}, 1 \mathrm{H}, J_{3,4}=\right.$ $2.9 \mathrm{~Hz}, \mathrm{H}-4), 5.31$ (dd, 1H, $\left.J_{2,3}=11.3 \mathrm{~Hz}, \mathrm{H}-3\right), 5.04$ (d, 1H, $\left.J_{1,2}=8.3 \mathrm{~Hz}, \mathrm{H}-1\right), 4.40$ (m, 1H, H-2), 4.26-4.12 (2dd, 2H, $\left.J_{6 \mathrm{a}, 6 \mathrm{~b}}=11.5 \mathrm{~Hz}, \mathrm{H}-6 \mathrm{a}, \mathrm{H}-6 \mathrm{~b}\right), 4.03$ (br dd, $1 \mathrm{H}, J_{5,6 \mathrm{a}}=$ $\left.J_{5,6 \mathrm{~b}}=6.5 \mathrm{~Hz}, \mathrm{H}-5\right), 3.76(\mathrm{~s}, 3 \mathrm{H}, \mathrm{Me}(\mathrm{OMP})), 2.18-2.00\left(3 \mathrm{~s}, 9 \mathrm{H}, \mathrm{CH}_{3}(\mathrm{Ac})\right) ;{ }^{13} \mathrm{C}-\mathrm{NMR}$ (75 MHz, $\left.\mathrm{CDCl}_{3}\right): \delta 170.7,170.6,170.3\left(\mathrm{COCH}_{3}\right), 158.0\left(\mathrm{q},{ }^{2} J_{\mathrm{C}, \mathrm{F}}=34.1 \mathrm{~Hz}, \mathrm{COCF}_{3}\right)$, 155.9, 151.0 (Ar-C), 118.8, $114.7(\mathrm{Ar}-\mathrm{CH}), 115.6\left(\mathrm{q},{ }^{1} J_{\mathrm{C}, \mathrm{F}}=287.0 \mathrm{~Hz}, \mathrm{COCF}_{3}\right), 100.4$ (C-1), 71.1 (C-5), 69.5 (C-3), 66.4 (C-4), 61.6 (C-6), 55.7 (Me(OMP)), 51.8 (C-2), 20.5 $\left(\mathrm{CH}_{3}(\mathrm{Ac})\right)$; HR MS: $m / z$ : calcd for $\mathrm{C}_{21} \mathrm{H}_{24} \mathrm{NO}_{10} \mathrm{~F}_{3} \mathrm{Na}$ : 530.1250 ; found: 530.1268 $[M+\mathrm{Na}]^{+}$.

\section{4-Methoxyphenyl 3,4,6-trihydroxy-2-deoxy-2-(trifluoroacetamido)- $\beta$-D-}

galactopyranoside (14): Compound 13 (2.8 g, $5.5 \mathrm{mmol}$ ) was dissolved in $\mathrm{MeOH}$ (39 $\mathrm{mL})$ and $\mathrm{NaOMe}(365 \mu \mathrm{L}, 2.17 \mathrm{M}$ solution in $\mathrm{MeOH})$ was added. After 50 min, Amberlite acidic resin was added until $\mathrm{pH}$ 7. The Amberlite resin was filtered off, and 
the solvent was removed in vacuo to give $\mathbf{1 4}$ (2.1 g, quantitative). TLC (16:1 $\mathrm{CH}_{2} \mathrm{Cl}_{2}$ $/ \mathrm{MeOH}) \mathrm{R}_{\mathrm{f}} 0.21 ;{ }^{1} \mathrm{H}$ NMR (300 MHz, MeOD): $\delta 6.97$ (m, 2H, Ar), 6.82 (m, 2H, Ar), $4.91\left(\mathrm{~d}, 1 \mathrm{H}, J_{1,2}=8.5 \mathrm{~Hz}, \mathrm{H}-1\right), 4.25\left(\mathrm{dd}, 1 \mathrm{H}, J_{2,3}=10.7 \mathrm{~Hz}, \mathrm{H}-2\right), 3.91\left(\mathrm{~d}, 1 \mathrm{H}, J_{3,4}=\right.$ 3.1 Hz, H-4), 3.87-3.71 (m, 6H, H-6a, H-6b, H-3, $\mathrm{CH}_{3}(\mathrm{OMP})$ ), 3.64 (dd, 1H, H-5); HR MS: $m / z$ : calcd for $\mathrm{C}_{15} \mathrm{H}_{18} \mathrm{NO}_{7} \mathrm{~F}_{3} \mathrm{Na}$ : 404.0933; found: $404.0923[M+\mathrm{Na}]^{+}$.

\section{4-Methoxyphenyl 4,6-O-benzylidene-2-deoxy-2-trifluoroacetamido- $\beta$-D-}

galactopyranoside (15): Benzaldehyde dimethyl acetal $(1.0 \mathrm{~mL}, 6.7 \mathrm{mmol})$ and $p$ toluenesulfonic acid $(0.08 \mathrm{~g}, 0.45 \mathrm{mmol})$ were added to a solution of $\mathbf{1 4}(1.7 \mathrm{~g}, 4.5$ $\mathrm{mmol}$ ) in $\mathrm{MeCN}(31 \mathrm{~mL})$. After stirring at room temperature for $4 \mathrm{~h}$, EtOAc was added and the mixture was washed with saturated aqueous $\mathrm{NaHCO}_{3}$. The organic phase was dried with $\mathrm{MgSO}_{4}$, filtered and concentrated in vacuo. Flash chromatography ( toluene/acetone $5: 1 \rightarrow 1: 1)$ gave $15(1.8 \mathrm{~g}, 87 \%)$. TLC (2:1 toluene/acetone) $\mathrm{R}_{\mathrm{f}} 0.43$;

$[\alpha]_{\mathrm{D}}^{20}-8.4^{\circ}(c 1.0$, acetone $) ;{ }^{1} \mathrm{H}-\mathrm{NMR}\left(400 \mathrm{MHz},\left(\mathrm{CD}_{3}\right)_{2} \mathrm{CO}\right): \delta 8.56\left(\mathrm{~d}, 1 \mathrm{H}, J_{2, \mathrm{NH}}=9.4\right.$ Hz, NH), 7.59 (m, 2H, Ar), 7.40 (m, 3H, Ar), 7.02 (m, 2H, Ar), 6.86 (m, 2H, Ar), 5.72 ( s, $1 \mathrm{H}, \mathrm{PhCHO}), 5.19$ (d, 1H, $\left.J_{1,2}=8.3 \mathrm{~Hz}, \mathrm{H}-1\right), 4.48-4.35$ (m, 3H, H-2, H-4, OH), 4.324.18 (m, 2H, H-6a, H-6b), 4.16-4.06 (m, 1H, H-3), 3.85 (s, 1H, H-5), 3.76 (s, 3H, Me (OMP)), ${ }^{13} \mathrm{C}-\mathrm{NMR}\left(100 \mathrm{MHz},\left(\mathrm{CD}_{3}\right)_{2} \mathrm{CO}\right): \delta 157.3\left(\mathrm{q},{ }^{2} J_{\mathrm{C}, \mathrm{F}}=36.0 \mathrm{~Hz}, C \mathrm{OCF}_{3}\right), 155.5-$ $115.5(\mathrm{Ar}), 116.3\left(\mathrm{q},{ }^{1} J_{\mathrm{C}, \mathrm{F}}=289.0 \mathrm{~Hz}, \mathrm{COCF}_{3}\right), 100.7(\mathrm{PhCHO}), 100.3(\mathrm{C}-1), 75.4(\mathrm{C}-$ 4), 69.8 (C-3), 68.8 (C-6), 66.9 (C-5), 55.0 (Me(OMP)), 53.8 (C-2); HR MS: m/z: calcd for $\mathrm{C}_{22} \mathrm{H}_{22} \mathrm{NO}_{7} \mathrm{~F}_{3} \mathrm{Na}$ : 492.1246; found: $492.1234[M+\mathrm{Na}]^{+}$.

\section{Dimethylthexylsilyl}

\section{3,4,6-tri- $O$-acetyl-2-deoxy-2-trifluoroacetamido- $\beta$-D-}

galactopyranoside (16): Benzylamine $(3.0 \mathrm{~mL}, 28 \mathrm{mmol})$ was added to a solution of $12(4.0 \mathrm{~g}, 9.0 \mathrm{mmol})$ in THF $(40 \mathrm{~mL})$. After stirring for $3 \mathrm{~h}$ at room temperature, the mixture was diluted with $\mathrm{CH}_{2} \mathrm{Cl}_{2}$, and washed with $1 \mathrm{M} \mathrm{HCl}$ and $\mathrm{H}_{2} \mathrm{O}$. The organic phase was dried over $\mathrm{Mg}_{2} \mathrm{SO}_{4}$, filtered and the solvent was removed under reduced 
pressure. The residue [TLC (3:2 hexane/EtOAc) $\mathrm{R}_{\mathrm{f}}$ 0.36] (3.9 $\mathrm{g}$ ) was dissolved in $\mathrm{CH}_{2} \mathrm{Cl}_{2}(19.5 \mathrm{~mL})$. Imidazole $(1.78 \mathrm{~g}, 26.2 \mathrm{mmol})$ and thexyldimethylsilyl chloride ( $2.30 \mathrm{~mL}, 11.7 \mathrm{mmol}$ ) were added. After $24 \mathrm{~h}$, the mixture was diluted with $\mathrm{CH}_{2} \mathrm{Cl}_{2}$ and washed with $\mathrm{H}_{2} \mathrm{O}$. The organic layer was dried over $\mathrm{MgSO}_{4}$, filtered, and the solvent was removed in vacuo. Flash chromatography on silica gel (3:1 hexane/EtOAc) afforded $16(2.43 \mathrm{~g}, 51 \%)$. TLC $\left(3: 1\right.$ hexane/EtOAc) $\mathrm{R}_{\mathrm{f}} 0.33 ;[\alpha]_{\mathrm{D}}^{20}-8.7^{\circ}\left(c 1.0, \mathrm{CHCl}_{3}\right)$; ${ }^{1} \mathrm{H}-\mathrm{NMR}\left(400 \mathrm{MHz}, \mathrm{CDCl}_{3}\right): \delta 6.71\left(\mathrm{~d}, 1 \mathrm{H}, J_{2, \mathrm{NH}}=9.3 \mathrm{~Hz}, \mathrm{NH}\right), 5.38\left(\mathrm{dd}, 1 \mathrm{H}, J_{3,4}=\right.$ $3.8 \mathrm{~Hz}, \mathrm{H}-4), 5.22\left(\mathrm{dd}, 1 \mathrm{H}, J_{3,4}=3.5 \mathrm{~Hz}, J_{2,3}=11.3 \mathrm{~Hz}, \mathrm{H}-3\right), 4.84\left(\mathrm{~d}, 1 \mathrm{H}, J_{1,2}=8.0\right.$ Hz, H-1), 4.29- 4.08 (m, 3H, H-2, H-6a, H-6b), 3.95 (m, 1H, H-5), 2.28-1.94 (3s, 9H, $\left.\mathrm{COCH}_{3}\right), 1.62\left(\mathrm{~m}, 1 \mathrm{H}, \mathrm{CH}\left(\mathrm{CH}_{3}\right)_{2}\right), 0.97-0.76\left(\mathrm{~m}, 12 \mathrm{H}, \mathrm{C}\left(\mathrm{CH}_{3}\right)_{2}\right.$ and $\left.\mathrm{CH}\left(\mathrm{CH}_{3}\right)_{2}\right), 0.29$ $0.07\left(2 \mathrm{~s}, 6 \mathrm{H}, \mathrm{Si}\left(\mathrm{CH}_{3}\right)_{2}\right) ;{ }^{13} \mathrm{C}-\mathrm{NMR}\left(100 \mathrm{MHz}, \mathrm{CDCl}_{3}\right): \delta 170.9,170.6,170.4\left(\mathrm{COCH}_{3}\right)$, $157.3\left(\mathrm{q},{ }^{2} J_{\mathrm{C}, \mathrm{F}}=36.9 \mathrm{~Hz}, C \mathrm{COCF}_{3}\right), 115.8\left(\mathrm{q},{ }^{1} J_{\mathrm{C}, \mathrm{F}}=290.0 \mathrm{~Hz}, \mathrm{COCF}_{3}\right), 96.0(\mathrm{C}-1), 71.0$ (C-5), 69.8 (C-3), 66.8 (C-4), 61.9 (C-6), 53.6 (C-2), 33.9, 24.8, 20.7, 20.5, 19.8, 18.4 (TDS, OAc), -2.0, -3.0 (TDS); HR MS: $m / z$ : calcd for $\mathrm{C}_{22} \mathrm{H}_{36} \mathrm{NO}_{9} \mathrm{~F}_{3} \mathrm{NaSi}$ : 566.2009; found: $566.1991[M+\mathrm{Na}]^{+}$.

\section{Dimethylthexylsilyl}

6-O-chloroacetyl-2-deoxy-2-trifluoroacetamido- $\beta$-D-

galactopyranoside (18): Compound 16 (2.2 g, $4.1 \mathrm{mmol})$ was dissolved in $\mathrm{MeOH}$ (31 $\mathrm{mL})$ and $\mathrm{NaOMe}(273 \mu \mathrm{L}, 2.17 \mathrm{M}$ solution in $\mathrm{MeOH})$ was added. After $50 \mathrm{~min}$, Amberlite acidic resin was added until $\mathrm{pH}$ 7. The Amberlite resin was filtered off, and the solvent was removed in vacuo to give the desired triol (1.7 $\mathrm{g}$, quantitative). An aliquot of this triol $(0.5 \mathrm{~g}, 1.2 \mathrm{mmol})$ was dissolved in anhydrous $\mathrm{CH}_{2} \mathrm{Cl}_{2}(45 \mathrm{~mL})$ and collidine $(4.5 \mathrm{~mL})$, and chloroacetic anhydride $(112 \mu \mathrm{L}, 1.4 \mathrm{mmol})$ was then added dropwise at $-60^{\circ} \mathrm{C}$. After stirring for $1 \mathrm{~h}$ at $-60^{\circ} \mathrm{C}$, the mixture was diluted with EtOAc and washed with $1 \mathrm{M} \mathrm{HCl}, \mathrm{NaHCO}_{3}$ and $\mathrm{H}_{2} \mathrm{O}$. The organic phase was dried $\left(\mathrm{MgSO}_{4}\right)$, filtered, and concentrated. Flash silica chromatography (3:2 hexane/EtOAc) afforded 18 
(454 mg, 77\%). TLC (3:2 hexane/EtOAc) $\mathrm{R}_{\mathrm{f}} 0.14 ;[\alpha]_{\mathrm{D}}^{20}+17^{\circ}(c 1.0, \mathrm{MeOH}) ;{ }^{1} \mathrm{H}-$ $\operatorname{NMR}\left(300 \mathrm{MHz}, \mathrm{CDCl}_{3}\right): \delta 7.31\left(\mathrm{~d}, 1 \mathrm{H}, J_{2, \mathrm{NH}}=7.9 \mathrm{~Hz}, \mathrm{NH}\right), 4.70\left(\mathrm{~d}, 1 \mathrm{H}, J_{1,2}=7.5\right.$ $\mathrm{Hz}, \mathrm{H}-1), 4.56-4.30$ (m, 2H, H-6a, H-6b), 4.10 (s, 2H, $\mathrm{COCH}_{2} \mathrm{Cl}$ ), 4.02-3.80 (m, 3H, H3, H-4, H-2), 3.75 (m, 1H, H-5), $1.58\left(\mathrm{~m}, 1 \mathrm{H}, \mathrm{CH}\left(\mathrm{CH}_{3}\right)_{2}\right), 0.98-0.70\left(\mathrm{~m}, 12 \mathrm{H}, \mathrm{C}\left(\mathrm{CH}_{3}\right)_{2}\right.$ and $\left.\mathrm{CH}\left(\mathrm{CH}_{3}\right)_{2}\right), 0.23-0.03\left(2 \mathrm{~s}, 6 \mathrm{H}, \mathrm{Si}\left(\mathrm{CH}_{3}\right)_{2}\right) ;{ }^{13} \mathrm{C}-\mathrm{NMR}\left(75 \mathrm{MHz}, \mathrm{CDCl}_{3}\right): \delta 167.7$ $\left(\mathrm{COCH}_{2} \mathrm{Cl}\right), 159.0\left(\mathrm{q},{ }^{2} J_{\mathrm{C}, \mathrm{F}}=36.8 \mathrm{~Hz}, C \mathrm{COCF}_{3}\right), 115.8\left(\mathrm{q},{ }^{1} J_{\mathrm{C}, \mathrm{F}}=285.0 \mathrm{~Hz}, C \mathrm{OCF}_{3}\right)$, 95.8 (C-1), 72.5 (C-5), 70.4, 68.3 (C-3, C-4), 65.1 (C-6), 55.9 (C-2), $40.5\left(\mathrm{CH}_{2} \mathrm{Cl}\right), 33.9$, 24.8, 19.8, 18.5 (Ac, TDS), $-2.0, \quad-4.0$ (TDS); ESI MS: $m / z$ : calcd for $\mathrm{C}_{18} \mathrm{H}_{31} \mathrm{ClF}_{3} \mathrm{NO}_{7} \mathrm{SiNa}$ : 516.2; found: $516.2[\mathrm{M}+\mathrm{Na}]^{+}$.

\section{4-Methoxyphenyl 4,6-O-di-tert-butylsilylene-2-deoxy-2-trifluoroacetamido- $\beta$-D-}

galactopyranoside (4): Compound 14 (0.66 g, 1.73 mmol) was dissolved in dry Py (30 $\mathrm{mL})$ and cooled $\left(0^{\circ} \mathrm{C}\right)$. Di-tert-butylsilyl bis(trifluoromethanesulfonate) (0.63 mL, 1.9 mmol) was added and the mixture was stirred at room temperature for $12 \mathrm{~min}$. The reaction was quenched with $\mathrm{MeOH}(3 \mathrm{~mL})$, diluted with EtOAc $(120 \mathrm{~mL})$, and washed with $1 \mathrm{M} \mathrm{HCl}$, saturated aqueous $\mathrm{NaHCO}_{3}$, and $\mathrm{H}_{2} \mathrm{O}$. The organic phase was dried $\left(\mathrm{MgSO}_{4}\right)$, filtered and concentrated to dryness. The residue was purified by column chromatography (toluene/EtOAc 5:1) to afford 4 (0.8 g, 89\%). TLC (toluene/EtOAc 3:1) $\mathrm{R}_{\mathrm{f}} 0.30 ;[\alpha]_{\mathrm{D}}^{20}-17^{\circ}\left(c\right.$ 1.0, $\left.\mathrm{CHCl}_{3}\right) ;{ }^{1} \mathrm{H}-\mathrm{NMR}\left(300 \mathrm{MHz}, \mathrm{CDCl}_{3}\right): \delta 6.92(\mathrm{~m}, 2 \mathrm{H}$, Ar), $6.87\left(\mathrm{~d}, 1 \mathrm{H}, J_{2, \mathrm{NH}}=9.1 \mathrm{~Hz}, \mathrm{NH}\right), 6.78(\mathrm{~m}, 2 \mathrm{H}, \mathrm{Ar}), 4.97\left(\mathrm{~d}, 1 \mathrm{H}, J_{1,2}=8.4 \mathrm{~Hz}, \mathrm{H}-1\right)$, 4.39 (d, 1H, J3,4 = 2.7 Hz, H-4), 4.24 (m, 2H, H-6a, H-6b), 4.09 (bq, 1H, H-2), 3.89 (dd, $\left.1 \mathrm{H}, J_{2,3}=10.5 \mathrm{~Hz}, \mathrm{H}-3\right), 3.75$ (s, 3H, Me(OMP)), 3.46 (bs, 1H, H-5), 2.68 (bs, 1H, OH), $1.09,1.06\left(2 \mathrm{~s}, 18 \mathrm{H}, \mathrm{C}\left(\mathrm{CH}_{3}\right)_{3}\right) ;{ }^{13} \mathrm{C}-\mathrm{NMR}\left(75 \mathrm{MHz}, \mathrm{CDCl}_{3}\right): \delta 158.2\left(\mathrm{q},{ }^{2} J_{\mathrm{C}, \mathrm{F}}=37.2 \mathrm{~Hz}\right.$, $\left.\mathrm{COCF}_{3}\right), 155.9,151.2(\mathrm{Ar}-\mathrm{C}), 119.7(\mathrm{Ar}-\mathrm{CH}), 115.9\left(\mathrm{q},{ }^{1} J_{\mathrm{C}, \mathrm{F}}=288.0 \mathrm{~Hz}, \mathrm{COCF}_{3}\right)$, 114.6 (Ar-CH), 100.2 (C-1), 72.0 (C-4), 71.5 (C-5), 70.7 (C-3), 66.9 (C-6), 55.7 


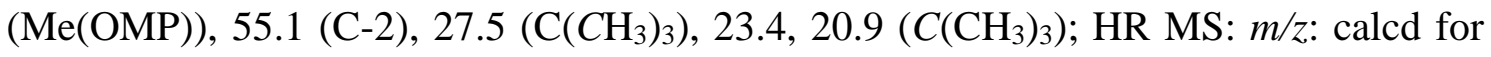
$\mathrm{C}_{23} \mathrm{H}_{34} \mathrm{~F}_{3} \mathrm{NO}_{7} \mathrm{SiNa}$ : 544.1954; found: $544.1968[M+\mathrm{Na}]^{+}$.

4-Methoxyphenyl 3-O-(benzyl 2-O-benzoyl-3-O-benzyl-4- $O$-levulinoyl- $\beta$-Dglucopyranosyluronate)-4,6-O-di-tert-butylsilylene-2-deoxy-2-trifluoroacetamido及-D-galactopyranoside (19): Acceptor 4 (0.18 g, $0.3 \mathrm{mmol})$ and glucuronic acid trichloroacetimidate $2(0.38 \mathrm{~g}, 0.5 \mathrm{mmol})$ were combined in a flask, coevaporated with toluene and dried under vacuum. The starting materials were disolved in dry $\mathrm{CH}_{2} \mathrm{Cl}_{2}(5$ $\mathrm{mL}$ ) and further dried by stirring over freshly activated $4 \AA$ molecular sieves for $15 \mathrm{~min}$. TMSOTf $(10 \mu \mathrm{L}, 0.05 \mathrm{mmol})$ was added at $0^{\circ} \mathrm{C}$. After $10 \mathrm{~min}$, the reaction was quenched with $\mathrm{Et}_{3} \mathrm{~N}(2.5 \mathrm{~mL})$ and filtered, and the solvent was removed under reduced pressure. Purification by flash cromathography (8:1 toluene/EtOAc) yielded 19 (345 mg, 91\%). TLC (5:1 toluene/EtOAc) $\mathrm{R}_{\mathrm{f}} 0.57 ;[\alpha]_{\mathrm{D}}^{20}+16.5^{\circ}\left(c 1.0, \mathrm{CH}_{2} \mathrm{Cl}_{2}\right) ;{ }^{1} \mathrm{H}-\mathrm{NMR}$ $\left(500 \mathrm{MHz}, \mathrm{CDCl}_{3}\right) \delta 8.01(\mathrm{~m}, 2 \mathrm{H}, \mathrm{Ar}), 7.62(\mathrm{~m}, 1 \mathrm{H}, \mathrm{Ar}), 7.47$ (m, 2H, Ar), 7.42-7.31 (m, 5H, Ar), 7.18-7.07 (m, 5H, Ar), 6.94 (m, 2H, Ar), 6.81(m, 2H, Ar), 5.41-5.30 (m, 4H, H-2B, H-1B, H-4B, H-1A), 5.18 (2d, 2H, $\left.\mathrm{CH}_{2}(\mathrm{Bn})\right)$,4.68-4.53 (m, 3H, H-4A, $\left.\mathrm{CH}_{2}(\mathrm{Bn})\right), 4.43\left(\mathrm{dd}, 1 \mathrm{H}, J_{2,3}=11.3 \mathrm{~Hz}, J_{3,4}=2.4 \mathrm{~Hz}, \mathrm{H}-3 \mathrm{~A}\right), 4.21-4.03$ (m, 4H, H-6aA, H-6bA, H-5B, H-2A), 3.86 (dd, 1H, $\left.J_{2,3}=J_{3,4}=8.7 \mathrm{~Hz}, \mathrm{H}-3 \mathrm{~B}\right), 3.78$ (s, 3H, Me(OMP)), 3.42 (s, 1H, H-5A), 2.62-2.22 (m, 4H, $\left.\mathrm{CH}_{2}(\mathrm{Lev})\right), 2.13$ (s, 3H, $\left.\mathrm{CH}_{3}(\mathrm{Lev})\right), 1.09,0.98$ (2s, 18H, C(CH3) $)$ ); ${ }^{13} \mathrm{C}-\mathrm{NMR}\left(100 \mathrm{MHz}, \mathrm{CDCl}_{3}\right): \delta 206.0(\mathrm{CO}(\mathrm{Lev})), 171.3,167.0$, $164.9(\mathrm{CO}(\mathrm{COOBn}, \mathrm{Bz}, \mathrm{Lev})), 157.6\left(\mathrm{q},{ }^{2} J_{\mathrm{C}, \mathrm{F}}=38.6 \mathrm{~Hz}, C \mathrm{OCF}_{3}\right), 155.8,151.1,137.2$, 134.6 (Ar-C), 133.5 (Ar-CH), 129.8-127.8 (Ar), $120.0(\mathrm{Ar}-\mathrm{CH}), 115.5\left(\mathrm{q},{ }^{1} J_{\mathrm{C}, \mathrm{F}}=288.5\right.$ $\left.\mathrm{Hz}, \mathrm{COCF}_{3}\right), 114.5$ (Ar-CH), 99.5, 99.4 (C-1B, C-1A), 79.6 (C-3B), 74.5 (C-3A), 74.2 $\left(\mathrm{CH}_{2}(\mathrm{Bn})\right), 73.2(\mathrm{C}-4 \mathrm{~A}), 72.5(\mathrm{C}-4 \mathrm{~B}$ or C-2B), $72.2(\mathrm{C}-5 \mathrm{~B}), 71.3(\mathrm{C}-5 \mathrm{~A}), 70.7(\mathrm{C}-2 \mathrm{~B}$ or C-4B), $68.1\left(\mathrm{CH}_{2}(\mathrm{Bn})\right), 67.0(\mathrm{C}-6 \mathrm{~A}), 55.6(\mathrm{Me}(\mathrm{OMP})), 53.7(\mathrm{C}-2 \mathrm{~A}), 37.6$ $\left(\mathrm{CH}_{2}(\mathrm{Lev})\right), 29.8\left(\mathrm{CH}_{3}(\mathrm{Lev})\right), 27.7,27.6,27.4\left(\mathrm{CH}_{2}(\mathrm{Lev}), \mathrm{C}\left(\mathrm{CH}_{3}\right)_{3}\right), 23.2,20.8$ 
$\left(\mathrm{C}\left(\mathrm{CH}_{3}\right)_{3}\right)$; HR MS: $m / z$ : calcd for $\mathrm{C}_{55} \mathrm{H}_{64} \mathrm{NO}_{16} \mathrm{~F}_{3} \mathrm{NaSi}$ : 1102.3844 ; found: 1102.3820 $[M+\mathrm{Na}]^{+}$.

4-Methoxyphenyl 3-O-(methyl

\section{3-O-benzyl-2,4-di- $O$-levulinoyl- $\alpha$-L-} idopyranosyluronate)-4,6-O-di-tert-butylsilylene-2-deoxy-2-trifluoroacetamido- $\beta$ D-galactopyranoside (20): Donor $3(0.92 \mathrm{~g}, 1.44 \mathrm{mmol})$ and acceptor $4(0.5 \mathrm{~g}, 0.96$ mmol) were dissolved in dry $\mathrm{CH}_{2} \mathrm{Cl}_{2}(12 \mathrm{~mL})$ in the presence of freshly activated $4 \AA$ molecular sieves. After stirring for $30 \mathrm{~min}$ at $0^{\circ} \mathrm{C}$, TMSOTf $(348 \mu \mathrm{L}$ of a $0.41 \mathrm{M}$ solution in dry $\mathrm{CH}_{2} \mathrm{Cl}_{2}$ ) was added under an argon atmosphere. After stirring for $15 \mathrm{~min}$ at $0^{\circ} \mathrm{C}$, the reaction mixture was neutralized with $\mathrm{Et}_{3} \mathrm{~N}$ and concentrated to dryness. The residue was purified by column chromatography (toluene/EtOAc 1:1) to afford 20 (756 mg, 79\%). TLC (toluene/EtOAc 1:1) $\mathrm{R}_{\mathrm{f}} 0.19 ;[\alpha]_{\mathrm{D}}^{20}-18^{\circ}\left(c 1.0, \mathrm{CHCl}_{3}\right) ;{ }^{1} \mathrm{H}-\mathrm{NMR}(300$ $\left.\mathrm{MHz}, \mathrm{CDCl}_{3}\right): \delta 7.34-7.27(\mathrm{~m}, 5 \mathrm{H}, \mathrm{Ar}), 7.00\left(\mathrm{~d}, 1 \mathrm{H}, J_{2, \mathrm{NH}}=7.2 \mathrm{~Hz}, \mathrm{NH}\right), 6.94(\mathrm{~m}, 2 \mathrm{H}$, Ar), 6.79 (m, 2H, Ar), 5.34 (d, 1H, $\left.J_{1,2}=8.3 \mathrm{~Hz}, \mathrm{H}-1 \mathrm{~A}\right), 5.28$ (bt, 1H, H-4B), 5.15 (bs, 1H, H-1B), $5.07\left(\mathrm{~d}, 1 \mathrm{H}, J_{4,5}=2.6 \mathrm{~Hz}, \mathrm{H}-5 \mathrm{~B}\right), 4.88(\mathrm{bt}, 1 \mathrm{H}, \mathrm{H}-2 \mathrm{~B}), 4.72(\mathrm{~m}, 2 \mathrm{H}$, $\left.\mathrm{CH}_{2}(\mathrm{Bn})\right), 4.54(\mathrm{bd}, 1 \mathrm{H}, \mathrm{H}-4 \mathrm{~A}), 4.35\left(\mathrm{dd}, 1 \mathrm{H}, J_{2,3}=11.0 \mathrm{~Hz}, J_{3,4}=2.3 \mathrm{~Hz}, \mathrm{H}-3 \mathrm{~A}\right), 4.20$ (m, 2H, H-6aA, H-6bA), 3.99 (m, 1H, H-2A), 3.80-3.75 (m, 7H, H-3B, Me(OMP), COOMe), 3.48 (bs, 1H, H-5A), 2.83-2.44 (m, 8H, $\mathrm{CH}_{2}(\mathrm{Lev})$ ), 2.16 (s, 6H, $\mathrm{CH}_{3}(\mathrm{Lev})$ ), 1.06, $0.97\left(2 \mathrm{~s}, 18 \mathrm{H}, \mathrm{C}\left(\mathrm{CH}_{3}\right)_{3}\right) ;{ }^{13} \mathrm{C}-\mathrm{NMR}\left(75 \mathrm{MHz} \mathrm{CDCl}_{3}\right): \delta 206.8,206.3(\mathrm{CO}(\mathrm{Lev}))$, 171.7, $171.6(\mathrm{CO}(\mathrm{Lev})), 168.9(\mathrm{COOMe}), 157.8\left(\mathrm{q},{ }^{2} J_{\mathrm{C}, \mathrm{F}}=36.8 \mathrm{~Hz}, C \mathrm{COCF}_{3}\right), 155.9$, 151.2, $138.0(\mathrm{Ar}-\mathrm{C}), 128.4,127.7,127.5,120.0(\mathrm{Ar}-\mathrm{CH}), 115.6\left(\mathrm{q},{ }^{1} J_{\mathrm{C}, \mathrm{F}}=287.8 \mathrm{~Hz}\right.$ $\left.\mathrm{COCF}_{3}\right), 114.6(\mathrm{Ar}-\mathrm{CH}), 100.3$ (C-1B), 99.1 (C-1A), 78.1 (C-3A), 73.3 (C-3B), 72.7, 72.6 (C-4A, $\left.\mathrm{CH}_{2}(\mathrm{Bn})\right), 71.3$ (C-5A), 68.6 (C-4B), 67.8 (C-2B), 67.4 (C-5B), 67.0 (C-6A), 55.7 (COOMe or Me(OMP)), 54.0 (C-2A), 52.6 (COOMe or Me(OMP)), 37.9, $37.8\left(\mathrm{CH}_{2}(\mathrm{Lev})\right), 29.9\left(\mathrm{CH}_{3}(\mathrm{Lev})\right), 28.0,27.7,27.4\left(\mathrm{CH}_{2}(\mathrm{Lev}), \mathrm{C}\left(\mathrm{CH}_{3}\right)_{3}\right), 23.3,20.9$ 
$\left(C\left(\mathrm{CH}_{3}\right)_{3}\right)$; HR MS: $m / z$ : calcd for $\mathrm{C}_{47} \mathrm{H}_{62} \mathrm{~F}_{3} \mathrm{NO}_{17} \mathrm{SiNa}$ : 1020.3637; found: 1020.3667 $[M+\mathrm{Na}]^{+}$.

4-Methoxyphenyl

idopyranosyluronate)-
3-O-(methyl

3-O-benzyl-2,4-di- $O$-levulinoyl- $\alpha$-L4,6-di- $O$-acetyl-2-deoxy-2-trifluoroacetamido- $\beta$-D-

galactopyranoside (22): An excess of $(\mathrm{HF})_{\mathrm{n}} \cdot \mathrm{Py}(1.26 \mathrm{~mL}, 48.3 \mathrm{mmol})$ was added at $0^{\circ} \mathrm{C}$ under an argon atmosphere to a solution of $\mathbf{2 0}(250 \mathrm{mg}, 0.25 \mathrm{mmol})$ in dry THF (5 $\mathrm{mL}$ ). After $23 \mathrm{~h}$ at $0^{\circ} \mathrm{C}$ the mixture was diluted with $\mathrm{CH}_{2} \mathrm{Cl}_{2}$ and washed with $\mathrm{H}_{2} \mathrm{O}$ and saturated $\mathrm{NaHCO}_{3}$ solution until neutral $\mathrm{pH}$. The organic layers were dried $\left(\mathrm{MgSO}_{4}\right)$, filtered and concentrated in vacuo to give 21 (209 mg, 97\%). TLC (toluene/acetone 3:2) $\mathrm{R}_{\mathrm{f}} 0.21 ;{ }^{1} \mathrm{H}-\mathrm{NMR}\left(300 \mathrm{MHz}, \mathrm{CDCl}_{3}\right): \delta 7.87\left(\mathrm{~d}, 1 \mathrm{H}, J_{2, \mathrm{NH}}=7.1 \mathrm{~Hz}, \mathrm{NH}\right), 7.36-7.24(\mathrm{~m}$, 5H, Ar), $6.96(\mathrm{~m}, 2 \mathrm{H}, \mathrm{Ar}), 6.80(\mathrm{~m}, 2 \mathrm{H}, \mathrm{Ar}), 5.47\left(\mathrm{~d}, 1 \mathrm{H}, J_{1,2}=8.5 \mathrm{~Hz}, \mathrm{H}-1 \mathrm{~A}\right), 5.23(\mathrm{~m}$, $2 \mathrm{H}, \mathrm{H}-4 \mathrm{~B}, \mathrm{H}-1 \mathrm{~B}), 4.98\left(\mathrm{t}, 1 \mathrm{H}, J_{1,2}=J_{2,3}=5.5 \mathrm{~Hz}, \mathrm{H}-2 \mathrm{~B}\right), 4.88\left(\mathrm{~d}, 1 \mathrm{H}, J_{4,5}=4.7 \mathrm{~Hz}, \mathrm{H}-\right.$ 5B), $4.68\left(\mathrm{~s}, 2 \mathrm{H}, \mathrm{CH}_{2}(\mathrm{Bn})\right), 4.48\left(\mathrm{dd}, 1 \mathrm{H}, J_{2,3}=10.8 \mathrm{~Hz}, J_{3,4}=3.1 \mathrm{~Hz}, \mathrm{H}-3 \mathrm{~A}\right), 4.20(\mathrm{~d}$, $1 \mathrm{H}, \mathrm{H}-4 \mathrm{~A}), 3.99\left(\mathrm{dd}, 1 \mathrm{H}, J_{5,6 \mathrm{a}}=6.6 \mathrm{~Hz}, J_{6 \mathrm{a}, 6 \mathrm{~b}}=11.8 \mathrm{~Hz}, \mathrm{H}-6 \mathrm{aA}\right), 3.95-3.82(\mathrm{~m}, 3 \mathrm{H}, \mathrm{H}-$ 2A, H-6bA, H-3B), 3.80, 3.76 (2s, 6H, Me(OMP), COOMe), 3.71 (bt, 1H, H-5A), 2.94$2.28\left(\mathrm{~m}, 8 \mathrm{H}, \mathrm{CH}_{2}(\mathrm{Lev})\right), 2.17$ (s, 6H, $\left.\mathrm{CH}_{3}(\mathrm{Lev})\right) ;{ }^{13} \mathrm{C}-\mathrm{NMR}\left(75 \mathrm{MHz}, \mathrm{CDCl}_{3}\right.$ ) (selected data from HSQC experiment): $\delta$ 129-127 (Ar-CH), 118.8, $114.2(\mathrm{Ar}-\mathrm{CH}), 99.0(\mathrm{C}-1 \mathrm{~B})$, 98.3 (C-1A), 75.9 (C-3A), 74.9 (C-3B), 74.0 (C-5A), $73.0\left(\mathrm{CH}_{2}(\mathrm{Bn})\right), 70.5(\mathrm{C}-2 \mathrm{~B}), 69.7$ (C-5B), 69.3 (C-4B), 68.4 (C-4A), 62.3 (C-6A), 55.3 (COOMe or Me(OMP)), 53.9 (C-2A), 52.3 (COOMe or $\mathrm{Me}(\mathrm{OMP})), 37.4\left(\mathrm{CH}_{2}(\mathrm{Lev})\right), 29.4\left(\mathrm{CH}_{3}(\mathrm{Lev})\right), 27.3$ $\left(\mathrm{CH}_{2}(\mathrm{Lev})\right.$; HR MS: $m / z$ : calcd for $\mathrm{C}_{39} \mathrm{H}_{46} \mathrm{~F}_{3} \mathrm{NO}_{17} \mathrm{Na}$ : 880.2616; found: 880.2617 $[M+\mathrm{Na}]^{+}$.

$21(152 \mathrm{mg}, 0.177 \mathrm{mmol})$ was dissolved in dry Py $(7 \mathrm{~mL})$, cooled $\left(0^{\circ} \mathrm{C}\right)$ and $\mathrm{Ac}_{2} \mathrm{O}(0.5$ $\mathrm{mL}$ ) was added. After stirring for $48 \mathrm{~h}$ at room temperature, the reaction mixture was diluted with $\mathrm{CH}_{2} \mathrm{Cl}_{2}$ and washed with $1 \mathrm{M} \mathrm{HCl}$ aqueous solution, saturated $\mathrm{NaHCO}_{3}$ 
aqueous solution and brine. The organic layers were dried $\left(\mathrm{MgSO}_{4}\right)$, filtered and concentrated in vacuo. The residue was purified by column chromatography (toluene/acetone 3:1) to afford 22 (149 $\mathrm{mg}, 89 \%)$. TLC (toluene/acetone 3:2) $\mathrm{R}_{\mathrm{f}}$ 0.49; $[\alpha]_{\mathrm{D}}^{20}-17^{\mathrm{o}}\left(c 1.0, \mathrm{CHCl}_{3}\right) ;{ }^{1} \mathrm{H}-\mathrm{NMR}\left(300 \mathrm{MHz}, \mathrm{CDCl}_{3}\right): \delta 7.77\left(\mathrm{~d}, 1 \mathrm{H}, J_{2, \mathrm{NH}}=7.4 \mathrm{~Hz}\right.$, $\mathrm{NH})$, 7.36-7.27 (m, 5H, Ar), $6.95(\mathrm{~m}, 2 \mathrm{H}, \mathrm{Ar}), 6.79(\mathrm{~m}, 2 \mathrm{H}, \mathrm{Ar}), 5.44\left(\mathrm{~d}, 1 \mathrm{H}, J_{1,2}=8.6\right.$ Hz, H-1A), 5.41(d, 1H, $\left.J_{3,4}=3.1 \mathrm{~Hz}, \mathrm{H}-4 \mathrm{~A}\right), 5.28$ (bt, 1H, H-4B), $5.01\left(\mathrm{~d}, 1 \mathrm{H}, J_{1,2}=3.9\right.$ Hz, H-1B), 4.92 (bt, 1H, $\left.J_{2,3}=5.5 \mathrm{~Hz}, \mathrm{H}-2 \mathrm{~B}\right), 4.88$ (d, 1H, $\left.J_{4,5}=3.3 \mathrm{~Hz}, \mathrm{H}-5 \mathrm{~B}\right), 4.63$ (q, $\left.2 \mathrm{H}, \mathrm{CH}_{2}(\mathrm{Bn})\right), 4.58\left(\mathrm{dd}, 1 \mathrm{H}, J_{2,3}=10.4 \mathrm{~Hz}, J_{3,4}=3.9 \mathrm{~Hz}, \mathrm{H}-3 \mathrm{~A}\right), 4.18-4.06(\mathrm{~m}, 2 \mathrm{H}$, H-6aA, H-6bA), 3.99 (bt, 1H, H-5A), 3.86 (m, 1H, H-2A), 3.80-3.75 (m, 7H, H-3B, Me(OMP), COOMe), 2.92-2.33 (m, 8H, $\mathrm{CH}_{2}(\mathrm{Lev})$ ), 2.19, 2.17 (2s, 6H, $\mathrm{CH}_{3}(\mathrm{Lev})$ ), 2.03, $1.92\left(2 \mathrm{~s}, 6 \mathrm{H}, \mathrm{CH}_{3}(\mathrm{Ac})\right) ;{ }^{13} \mathrm{C}-\mathrm{NMR}\left(75 \mathrm{MHz}, \mathrm{CDCl}_{3}\right): \delta 207.8,206.3(\mathrm{CO}(\mathrm{Lev}))$, 171.5, 171.4, 170.5, $170.4(\mathrm{CO}(\mathrm{Lev}, \mathrm{Ac})), 168.7(\mathrm{COOMe}), 157.9\left(\mathrm{q},{ }^{2} J_{\mathrm{C}, \mathrm{F}}=37.5 \mathrm{~Hz}\right.$, $\left.\mathrm{COCF}_{3}\right), 155.9,151.1,137.6(\mathrm{Ar}-\mathrm{C}), 128.5,127.9,119.1(\mathrm{Ar}-\mathrm{CH}), 115.6\left(\mathrm{q},{ }^{1} J_{\mathrm{C}, \mathrm{F}}=\right.$ 288.1 Hz, $\left.\mathrm{COCF}_{3}\right), 114.6$ (Ar-CH), 100.3 (C-1B), 99.0 (C-1A), 75.0 (C-3B), 73.7 (C3A), $73.2\left(\mathrm{CH}_{2}(\mathrm{Bn})\right), 71.3(\mathrm{C}-5 \mathrm{~A}), 69.9$ (C-4B), 69.4 (C-2B), 68.8 (C-4A), 68.6 (C-5B), 61.7 (C-6A), 55.7 (COOMe or Me(OMP)), 55.2 (C-2A), 52.5 (COOMe or Me(OMP)), 37.9, $37.7\left(\mathrm{CH}_{2}(\mathrm{Lev})\right), 29.8\left(\mathrm{CH}_{3}(\mathrm{Lev})\right), 27.9,27.6\left(\mathrm{CH}_{2}(\mathrm{Lev}), 20.7,20.4\left(\mathrm{CH}_{3}(\mathrm{Ac})\right)\right.$; HR MS: $m / z$ : calcd for $\mathrm{C}_{43} \mathrm{H}_{50} \mathrm{~F}_{3} \mathrm{NO}_{19} \mathrm{Na}$ : 964.2827; found: $964.2841[M+\mathrm{Na}]^{+}$.

\section{3-O-(Methyl 3-O-benzyl-2,4-di- $O$-levulinoyl- $\alpha$-L-idopyranosyluronate)-4,6-di- $O$ -} acetyl-2-deoxy-2-trifluoroacetamido- $\boldsymbol{\alpha}, \boldsymbol{\beta}$-D-galactopyranose (23): CAN (0.75 mL of a $0.63 \mathrm{M}$ solution in $\left.\mathrm{H}_{2} \mathrm{O}\right)$ was added to a solution of $22(149 \mathrm{mg}, 0.158 \mathrm{mmol})$ in toluene/MeCN $(1: 6 ; 5.25 \mathrm{~mL})$, and the mixture was vigorously stirred for $1 \mathrm{~h} 20 \mathrm{~min}$ at $0^{\circ} \mathrm{C}$. It was then diluted with EtOAc, washed with $\mathrm{H}_{2} \mathrm{O}$, saturated aqueous $\mathrm{NaHCO}_{3}$, and $\mathrm{H}_{2} \mathrm{O}$. The organic phase was dried $\left(\mathrm{MgSO}_{4}\right)$, filtered and concentrated to dryness. The residue was purified by column chromatography (toluene/acetone 5:2) to afford $\mathbf{2 3}$ 
(100 mg, 76\%) as a mixture of $\alpha / \beta$ anomers. TLC (toluene/acetone 3:2) $\mathrm{R}_{\mathrm{f}} 0.34,0.31$; ${ }^{1} \mathrm{H}-\mathrm{NMR}\left(300 \mathrm{MHz}, \mathrm{CDCl}_{3}\right.$ ) (data for $\alpha$ anomer): $\delta$ 7.39-7.26 (m, 5H, Ar), $6.73(\mathrm{~d}, 1 \mathrm{H}$, $\left.J_{2, \mathrm{NH}}=9.3 \mathrm{~Hz}, \mathrm{NH}\right), 5.40\left(\mathrm{~d}, 1 \mathrm{H}, J_{3,4}=3.0 \mathrm{~Hz}, \mathrm{H}-4 \mathrm{~A}\right), 5.37\left(\mathrm{~d}, 1 \mathrm{H}, J_{1,2}=3.1 \mathrm{~Hz}, \mathrm{H}-\right.$ 1A), 5.20 (bt, 1H, H-4B), 5.06 (bs, 1H, H-1B), 4.92 (d, 1H, $\left.J_{4,5}=2.3 \mathrm{~Hz}, \mathrm{H}-5 \mathrm{~B}\right), 4.80$ (m, 1H, H-2B), 4.63 (q, 2H, $\left.\mathrm{CH}_{2}(\mathrm{Bn})\right), 4.58$ (m, 1H, H-2A), 4.36 (bt, 1H, H-5A), 4.13 $\left(\mathrm{dd}, 1 \mathrm{H}, J_{2,3}=10.8 \mathrm{~Hz}, \mathrm{H}-3 \mathrm{~A}\right), 4.07\left(\mathrm{dd}, 1 \mathrm{H}, J_{5,6 \mathrm{a}}=5.3 \mathrm{~Hz}, J_{6 \mathrm{a}, 6 \mathrm{~b}}=11.5 \mathrm{~Hz}, \mathrm{H}-6 \mathrm{aA}\right)$, $3.97\left(\mathrm{dd}, 1 \mathrm{H}, J_{5,6 \mathrm{~b}}=7.0 \mathrm{~Hz}, \mathrm{H}-6 \mathrm{bA}\right), 3.81(\mathrm{~s}, 3 \mathrm{H}, \mathrm{COOMe}), 3.75$ (bt, 1H, H-3B), 3.27 (bs, 1H, OH), 2.84-2.48 (m, 8H, $\left.\mathrm{CH}_{2}(\mathrm{Lev})\right), 2.18$ (s, 6H, $\left.\mathrm{CH}_{3}(\mathrm{Lev})\right), 2.03,1.68$ (2s, 6H, $\left.\mathrm{CH}_{3}(\mathrm{Ac})\right) ;{ }^{13} \mathrm{C}-\mathrm{NMR}\left(75 \mathrm{MHz}, \mathrm{CDCl}_{3}\right.$ ) (data for $\alpha$ anomer): $\delta$ 207.5, $206.4(\mathrm{CO}(\mathrm{Lev})$ ), 171.7, 171.5, 170.7 (CO(Lev, Ac)), $169.4(\mathrm{COOMe}), 157.8\left(\mathrm{q},{ }^{2} J_{\mathrm{C}, \mathrm{F}}=37.3 \mathrm{~Hz}, C \mathrm{COCF}_{3}\right)$, $137.4(\mathrm{Ar}-\mathrm{C}), 128.5,128.2,128.0(\mathrm{Ar}-\mathrm{CH}), 115.9\left(\mathrm{q},{ }^{1} J_{\mathrm{C}, \mathrm{F}}=288.6 \mathrm{~Hz}, \mathrm{COCF}_{3}\right), 100.4$ (C-1B), 91.8 (C-1A), 75.0 (C-3A), $72.8\left(\mathrm{CH}_{2}(\mathrm{Bn})\right), 72.5$ (C-3B), 69.0 (C-4A), 68.4 (C4B), 67.7 (C-5A), 67.2 (C-2B), 67.1 (C-5B), 62.3 (C-6A), 52.7 (COOMe), 49.9 (C-2A), $37.9\left(\mathrm{CH}_{2}(\mathrm{Lev})\right), 30.0\left(\mathrm{CH}_{3}(\mathrm{Lev})\right), 27.9\left(\mathrm{CH}_{2}(\mathrm{Lev}), 20.9,20.3\left(\mathrm{CH}_{3}(\mathrm{Ac})\right)\right.$; HR MS: $m / z$ : calcd for $\mathrm{C}_{36} \mathrm{H}_{44} \mathrm{~F}_{3} \mathrm{NO}_{18} \mathrm{Na}$ : 858.2408; found: $858.2403[M+\mathrm{Na}]^{+}$.

$O$-[3-O-(Methyl 3-O-benzyl-2,4-di- $O$-levulinoyl- $\alpha$-L-idopyranosyluronate)-4,6-di- $O$ acetyl-2-deoxy-2-trifluoroacetamido- $\alpha, \beta$-D-galactopyranosyl] trichloroacetimidate (24): Trichloroacetonitrile $(180 \mu \mathrm{L}, 1.8 \mathrm{mmol})$ and catalytic DBU $(107 \mu \mathrm{L}$ of a $0.084 \mathrm{M}$ solution in dry $\mathrm{CH}_{2} \mathrm{Cl}_{2}$ ) were added to a solution of $23(100 \mathrm{mg}, 0.12 \mathrm{mmol})$ in dry $\mathrm{CH}_{2} \mathrm{Cl}_{2}(2 \mathrm{~mL})$. After stirring for $13 \mathrm{~h}$ at room temperature, the reaction mixture was concentrated to dryness. The residue was purified by flash chromatography (toluene/acetone $\left.5: 2+1 \% \mathrm{Et}_{3} \mathrm{~N}\right)$ to afford $24(99 \mathrm{mg}, 84 \%)$ as a mixture of $\alpha / \beta$ anomers. TLC (toluene/acetone 5:2) $\mathrm{R}_{\mathrm{f}} 0.42(\alpha) ;{ }^{1} \mathrm{H}-\mathrm{NMR}\left(400 \mathrm{MHz}, \mathrm{CDCl}_{3}\right.$ ) (data for $\alpha$ anomer): $\delta 8.82(\mathrm{~s}, 1 \mathrm{H}, \mathrm{NH}(\mathrm{TCA})), 7.36-7.26(\mathrm{~m}, 5 \mathrm{H}, \mathrm{Ar}), 6.88\left(\mathrm{~d}, 1 \mathrm{H}, J_{2, \mathrm{NH}}=9.2\right.$ Hz, NH(TFA)), 6.39 (d, 1H, $\left.J_{1,2}=3.7 \mathrm{~Hz}, \mathrm{H}-1 \mathrm{~A}\right), 5.47$ (bd, 1H, $\left.J_{3,4}=2.9 \mathrm{~Hz}, \mathrm{H}-4 \mathrm{~A}\right)$, 
5.18 (bt, 1H, H-4B), 5.13 (bd, 1H, H-1B), 4.87 (d, 1H, J4,5 = 2.7 Hz, H-5B), 4.78 (m, 1H, H-2B), 4.74 (m, 1H, H-2A), 4.61 (q, 2H, $\mathrm{CH}_{2}(\mathrm{Bn})$ ), 4.28 (bt, 1H, H-5A), 4.20 (dd, $\left.1 \mathrm{H}, J_{2,3}=10.9 \mathrm{~Hz}, \mathrm{H}-3 \mathrm{~A}\right), 4.08\left(\mathrm{dd}, 1 \mathrm{H}, J_{5,6 \mathrm{a}}=5.9 \mathrm{~Hz}, J_{6 \mathrm{a}, 6 \mathrm{~b}}=11.6 \mathrm{~Hz}, \mathrm{H}-6 \mathrm{aA}\right), 3.94$ $\left(\mathrm{dd}, 1 \mathrm{H}, J_{5,6 \mathrm{~b}}=7.1 \mathrm{~Hz}, \mathrm{H}-6 \mathrm{bA}\right), 3.78$ (s, 3H, COOMe), 3.74 (bt, 1H, H-3B), 2.80-2.46 (m, 8H, $\left.\mathrm{CH}_{2}(\mathrm{Lev})\right), 2.15,2.13\left(2 \mathrm{~s}, 6 \mathrm{H}, \mathrm{CH}_{3}(\mathrm{Lev})\right), 1.96,1.72$ (2s, 6H, $\left.\mathrm{CH}_{3}(\mathrm{Ac})\right)$; ${ }^{13} \mathrm{C}-\mathrm{NMR}\left(100 \mathrm{MHz}, \mathrm{CDCl}_{3}\right.$ ) (data for $\alpha$ anomer): $\delta$ 206.8, $206.2(\mathrm{CO}(\mathrm{Lev})), 171.6$, 170.4, $170.3(\mathrm{CO}(\mathrm{Lev}, \mathrm{Ac})), 168.9(\mathrm{COOMe}), 160.3(\mathrm{C}=\mathrm{NH}), 157.7\left(\mathrm{q},{ }^{2} J_{\mathrm{C}, \mathrm{F}}=38.0 \mathrm{~Hz}\right.$, $\left.\mathrm{COCF}_{3}\right), 137.3(\mathrm{Ar}-\mathrm{C}), 128.5,128.1,128.0(\mathrm{Ar}-\mathrm{CH}), 115.7\left(\mathrm{q},{ }^{1} J_{\mathrm{C}, \mathrm{F}}=289.0 \mathrm{~Hz}\right.$, $\left.\mathrm{COCF}_{3}\right), 100.6(\mathrm{C}-1 \mathrm{~B}), 94.9(\mathrm{C}-1 \mathrm{~A}), 90.7\left(\mathrm{CCl}_{3}\right), 75.1(\mathrm{C}-3 \mathrm{~A}), 72.7\left(\mathrm{CH}_{2}(\mathrm{Bn})\right), 72.5$ (C-3B), 69.9 (C-5A), 68.2 (C-4A), 68.1 (C-4B), 67.6 (C-2B), 67.2 (C-5B), 61.7 (C-6A), 52.7 (COOMe), 49.5 (C-2A), 37.8, $37.6\left(\mathrm{CH}_{2}(\mathrm{Lev})\right), 29.8\left(\mathrm{CH}_{3}(\mathrm{Lev})\right), 27.9,27.7$ $\left(\mathrm{CH}_{2}(\mathrm{Lev}), 20.6,20.1\left(\mathrm{CH}_{3}(\mathrm{Ac})\right)\right.$; ESI MS: $\mathrm{m} / z$ : calcd for $\mathrm{C}_{38} \mathrm{H}_{44} \mathrm{Cl}_{3} \mathrm{~F}_{3} \mathrm{~N}_{2} \mathrm{O}_{18} \mathrm{Na}$ : 1001.2; found: $1001.1[M+\mathrm{Na}]^{+}$.

\section{4-Methoxyphenyl 3- $O$-(benzyl 2- $O$-benzoyl-3- $O$-benzyl- $\beta$-D-}

\section{glucopyranosyluronate)-4,6-O-di-tert-butylsilylene-2-deoxy-2-trifluoroacetamido-}

$\boldsymbol{\beta}$-D-galactopyranoside (25): Compound 19 (0.25 g, $0.23 \mathrm{mmol})$ was dissolved in $\mathrm{CH}_{2} \mathrm{Cl}_{2}$ (4 mL), and hydrazine monohydrate $(2 \mathrm{~mL}$ of a $0.25 \mathrm{M}$ solution in $\mathrm{Py} / \mathrm{AcOH}$, 3:2) was added. After stirring at room temperature for $2 \mathrm{~h}$, the reaction mixture was quenched with acetone $(1 \mathrm{~mL})$, diluted with $\mathrm{CH}_{2} \mathrm{Cl}_{2}$, washed with $\mathrm{HCl}(1 \mathrm{M})$, saturated aqueous $\mathrm{NaHCO}_{3}$ and $\mathrm{H}_{2} \mathrm{O}$, dried with $\mathrm{MgSO}_{4}$, filtered and concentrated in vacuo. The residue was purified by flash chromatography on silica gel (9:1 toluene/EtOAc) to give $25(0.19 \mathrm{~g}, 84 \%)$. TLC (5:1 toluene/ EtOAc) $\mathrm{R}_{\mathrm{f}} 0.43 ;[\alpha]_{\mathrm{D}}^{20}+6.1^{\circ}\left(c 1.0, \mathrm{CH}_{2} \mathrm{Cl}_{2}\right)$; ${ }^{1} \mathrm{H}-\mathrm{NMR}\left(500 \mathrm{MHz}, \mathrm{CDCl}_{3}\right) \delta 8.00(\mathrm{~m}, 2 \mathrm{H}, \mathrm{Ar}), 7.61(\mathrm{~m}, 1 \mathrm{H}, \mathrm{Ar}), 7.46(\mathrm{~m}, 2 \mathrm{H}, \mathrm{Ar})$, 7.43- $7.31(\mathrm{~m}, 5 \mathrm{H}, \mathrm{Ar}), 7.23-7.12(\mathrm{~m}, 5 \mathrm{H}, \mathrm{Ar}), 6.98\left(\mathrm{~d}, 1 \mathrm{H}, J_{2, \mathrm{NH}}=6.5 \mathrm{~Hz}, \mathrm{NH}\right), 6.92$ (m, 2H, Ar), 6.81(m, 2H, Ar), $5.44\left(\mathrm{~d}, 1 \mathrm{H}, J_{1,2}=8.2 \mathrm{~Hz}, \mathrm{H}-1 \mathrm{~A}\right), 5.39$ (d, 1H, $\left.\mathrm{CH}_{2}(\mathrm{Bn})\right)$, 
$5.34\left(\mathrm{~d}, 1 \mathrm{H}, J_{1,2}=7.9 \mathrm{~Hz}, \mathrm{H}-1 \mathrm{~B}\right), 5.29(\mathrm{dd}, 1 \mathrm{H}, \mathrm{H}-2 \mathrm{~B}), 5.20\left(\mathrm{~d}, 1 \mathrm{H}, \mathrm{CH}_{2}(\mathrm{Bn})\right), 4.77(2 \mathrm{~d}$, 2H, $\left.\mathrm{CH}_{2}(\mathrm{Bn})\right), 4.62(\mathrm{~d}, 1 \mathrm{H}, \mathrm{H}-4 \mathrm{~A}), 4.44\left(\mathrm{dd}, 1 \mathrm{H}, J_{2,3}=11.3 \mathrm{~Hz}, J_{3,4}=2.4 \mathrm{~Hz}, \mathrm{H}-3 \mathrm{~A}\right)$, 4.19-4.05 (m, 2H, H-6aA, H-4B), 4.03-4.91 (m, 3H, H-6bA, H-5B, H-2A), 3.78 (s, 3H, $\operatorname{Me}(\mathrm{OMP})), 3.69\left(\mathrm{dd}, 1 \mathrm{H}, J_{2,3}=J_{3,4}=9.2 \mathrm{~Hz}, \mathrm{H}-3 \mathrm{~B}\right), 3.35$ (s, 1H, H-5A), 1.08, 1.00 (2s, $\left.18 \mathrm{H}, \mathrm{C}\left(\mathrm{CH}_{3}\right)_{3}\right) ;{ }^{13} \mathrm{C}-\mathrm{NMR}\left(100 \mathrm{MHz}, \mathrm{CDCl}_{3}\right): \delta 169.0,165.0(\mathrm{CO}(\mathrm{COOBn}, \mathrm{Bz})), 157.7$ $\left(\mathrm{q},{ }^{2} J_{\mathrm{C}, \mathrm{F}}=37.1 \mathrm{~Hz}, \mathrm{COCF}_{3}\right), 155.9,151.1,137.6,134.4(\mathrm{Ar}-\mathrm{C}), 133.5(\mathrm{Ar}-\mathrm{CH}), 129.9-$ $127.8(\mathrm{Ar}), 120.2(\mathrm{Ar}-\mathrm{CH}), 115.4\left(\mathrm{q},{ }^{1} J_{\mathrm{C}, \mathrm{F}}=286.4 \mathrm{~Hz}, \mathrm{COCF}_{3}\right), 114.5(\mathrm{Ar}-\mathrm{CH}), 100.3$ (C-1B), 99.0 (C-1A), 81.0 (C-3B), 74.9 (C-3A), $74.7\left(\mathrm{CH}_{2}(\mathrm{Bn})\right), 73.8$ (C-5B), 73.4 (C4A), 72.5 (C-2B), 72.2 (C-4B), 71.3 (C-5A), $68.0\left(\mathrm{CH}_{2}(\mathrm{Bn})\right), 66.9(\mathrm{C}-6 \mathrm{~A}), 55.6$

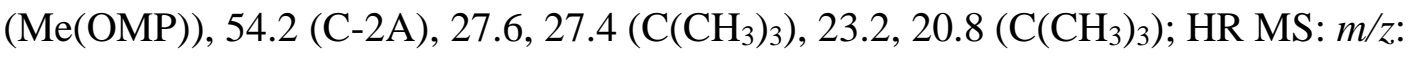
calcd for $\mathrm{C}_{50} \mathrm{H}_{58} \mathrm{NO}_{14} \mathrm{~F}_{3} \mathrm{NaSi}$ : 1004.3476; found: $1004.3511[M+\mathrm{Na}]^{+}$.

4-Methoxyphenyl $\quad O$-(methyl 3- $O$-benzyl-2,4-di- $O$-levulinoyl- $\alpha$-Lidopyranosyluronate)-(1 $\rightarrow 3)-O$-(4,6-di- $O$-acetyl-2-deoxy-2-trifluoroacetamido- $\beta$-Dgalactopyranosyl)-( $1 \rightarrow 4)-O-($ benzyl 2-O-benzoyl-3- $O$-benzyl- $\beta$-Dglucopyranosyluronate)-(1 $\rightarrow 3$ )-4,6-O-di-tert-butylsilylene-2-deoxy-2trifluoroacetamido- $\beta$-D-galactopyranoside (26): Donor $24(52 \mathrm{mg}, 53 \mu \mathrm{mol})$ and acceptor 25 (35 mg, $35 \mu \mathrm{mol})$ were dissolved in dry $\mathrm{CH}_{2} \mathrm{Cl}_{2}(1.0 \mathrm{~mL})$ in the presence of freshly activated $4 \AA$ molecular sieves. After stirring for 30 min, TMSOTf (115 $\mu \mathrm{L}$ of a $0.092 \mathrm{M}$ solution in dry $\mathrm{CH}_{2} \mathrm{Cl}_{2}$ ) was added under an argon atmosphere at $0^{\circ} \mathrm{C}$. After stirring for $15 \mathrm{~min}$ at $0^{\circ} \mathrm{C}$, the reaction mixture was neutralized with $\mathrm{Et}_{3} \mathrm{~N}$ and concentrated to dryness. The residue was purified by column chromatography (toluene/acetone 3:1) to afford 26 (47 $\mathrm{mg}, 74 \%$ ). TLC (toluene/acetone 3:1) $\mathrm{R}_{\mathrm{f}} 0.32$; $[\alpha]_{\mathrm{D}}^{20}-5^{\circ}\left(c 1.0, \mathrm{CHCl}_{3}\right) ;{ }^{1} \mathrm{H}-\mathrm{NMR}\left(400 \mathrm{MHz}, \mathrm{CDCl}_{3}\right): \delta 7.93(\mathrm{~d}, 2 \mathrm{H}, \mathrm{Ar}), 7.57(\mathrm{t}, 1 \mathrm{H}$, Ar), 7.47-7.22 (m, 12H, Ar), $7.06(\mathrm{~m}, 5 \mathrm{H}, \mathrm{Ar}), 6.96\left(\mathrm{~d}, 1 \mathrm{H}, J_{2, \mathrm{NH}}=8.7 \mathrm{~Hz}, \mathrm{NH}\right), 6.90$ (m, 2H, Ar), $6.83\left(\mathrm{~d}, 1 \mathrm{H}, J_{2, \mathrm{NH}}=7.0 \mathrm{~Hz}, \mathrm{NH}\right), 6.78(\mathrm{~m}, 2 \mathrm{H}, \mathrm{Ar}), 5.34\left(\mathrm{~d}, 1 \mathrm{H}, \mathrm{CH}_{2}(\mathrm{Bn})\right)$, 
$5.33\left(\mathrm{~d}, 1 \mathrm{H}, J_{1,2}=8.6 \mathrm{~Hz}, \mathrm{H}-1 \mathrm{~A}\right), 5.25(\mathrm{~m}, 3 \mathrm{H}, \mathrm{H}-1 \mathrm{~B}, \mathrm{H}-2 \mathrm{~B}, \mathrm{H}-4 \mathrm{D}), 5.19(\mathrm{~d}, 1 \mathrm{H}$, $\left.\mathrm{CH}_{2}(\mathrm{Bn})\right), 5.13$ (bd, 1H, H-4C), 4.98 (bs, 1H, H-1D), 4.96 (d, 1H, J4,5 = 2.4 Hz, H-5D), 4.80 (m, 2H, H-2D, $\left.\mathrm{CH}_{2}(\mathrm{Bn})\right), 4.64\left(\mathrm{~m}, 2 \mathrm{H}, \mathrm{CH}_{2}(\mathrm{Bn})\right), 4.57$ (d, 1H, J3,4 $=2.5 \mathrm{~Hz}, \mathrm{H}-$ 4A), $4.51\left(\mathrm{~d}, 1 \mathrm{H}, \mathrm{CH}_{2}(\mathrm{Bn})\right), 4.37\left(\mathrm{dd}, 1 \mathrm{H}, J_{2,3}=11.3 \mathrm{~Hz}, \mathrm{H}-3 \mathrm{~A}\right), 4.18\left(\mathrm{~d}, 1 \mathrm{H}, J_{1,2}=8.5\right.$ Hz, H-1C), 4.12 (bd, 1H, J6a,6b = 12.3 Hz, H-6aA), 4.04 (m, 3H, H-6bA, H-4B, H-2C), $3.96\left(\mathrm{~d}, 1 \mathrm{H}, J_{4,5}=9.3 \mathrm{~Hz}, \mathrm{H}-5 \mathrm{~B}\right), 3.93(\mathrm{~m}, 1 \mathrm{H}, \mathrm{H}-2 \mathrm{~A}), 3.81$ (m, 4H, H-6aC, Me(OMP) or COOMe), 3.75 (s, 3H, Me(OMP) or COOMe), 3.74-3.66 (m, 3H, H-3D, H-6bC, H3B), $3.61\left(\mathrm{dd}, 1 \mathrm{H}, J_{2,3}=10.6 \mathrm{~Hz}, J_{3,4}=3.4 \mathrm{~Hz}, \mathrm{H}-3 \mathrm{C}\right), 3.33$ (m, 2H, H-5A, H-5C), 2.832.50 (m, 8H, $\left.\mathrm{CH}_{2}(\mathrm{Lev})\right), 2.19,2.18$ (2s, 6H, $\left.\mathrm{CH}_{3}(\mathrm{Lev})\right), 1.97,1.61$ (2s, 6H, $\left.\mathrm{CH}_{3}(\mathrm{Ac})\right)$, 1.06, $1.02\left(2 \mathrm{~s}, 18 \mathrm{H}, \mathrm{C}\left(\mathrm{CH}_{3}\right)_{3}\right) ;{ }^{13} \mathrm{C}-\mathrm{NMR}\left(100 \mathrm{MHz}, \mathrm{CDCl}_{3}\right): \delta 206.9,206.3(\mathrm{CO}(\mathrm{Lev}))$, 171.7, 171.5, 170.4, 170.3, 168.9, 168.8, 165.0 (CO(Lev), $\mathrm{CO}(\mathrm{Ac}), \mathrm{CO}(\mathrm{Bz}), \mathrm{COOBn}$, COOMe), $158.1\left(\mathrm{q},{ }^{2} J_{\mathrm{C}, \mathrm{F}}=36.4 \mathrm{~Hz}, C \mathrm{COCF}_{3}\right), 157.7\left(\mathrm{q},{ }^{2} J_{\mathrm{C}, \mathrm{F}}=36.4 \mathrm{~Hz}, C O \mathrm{CF}_{3}\right), 156.0$, 151.1, 137.8, 137.6, 134.7 (Ar-C), 133.4, 129.9, 129.7, 129.6, 129.4, 129.1, 128.7, 128.5, 128.1, 127.9, 127.6, $120.3(\mathrm{Ar}-\mathrm{C}, \mathrm{Ar}-\mathrm{CH}), 116.0\left(\mathrm{q},{ }^{1} J_{\mathrm{C}, \mathrm{F}}=286.3 \mathrm{~Hz}, \mathrm{COCF}_{3}\right)$, $115.5\left(\mathrm{q},{ }^{1} J_{\mathrm{C}, \mathrm{F}}=287.9 \mathrm{~Hz}, \mathrm{COCF}_{3}\right), 114.6(\mathrm{Ar}-\mathrm{CH}), 100.4(\mathrm{C}-1 \mathrm{~B}), 100.3(\mathrm{C}-1 \mathrm{D}), 99.7$ (C-1C), 99.3 (C-1A), 80.1 (C-3B), 78.0 (C-4B), 76.4 (C-3C), 75.5 (C-3A), 75.1 $\left(\mathrm{CH}_{2}(\mathrm{Bn})\right), 74.3$ (C-5B), $73.3(\mathrm{C}-4 \mathrm{~A}), 73.0(\mathrm{C}-3 \mathrm{D}), 72.6\left(\mathrm{CH}_{2}(\mathrm{Bn})\right), 72.4(\mathrm{C}-2 \mathrm{~B}), 71.4$, 71.1 (C-5A, C-5C), 68.5, $68.4\left(\mathrm{CH}_{2}(\mathrm{Bn}), \mathrm{C}-4 \mathrm{D}\right), 67.8$ (C-4C), 67.5 (C-2D), 67.0 (C-5D, C-6A), 61.0 (C-6C), 55.7 (COOMe or Me(OMP)), 54.2 (C-2A), 53.5 (C-2C), 52.6 (COOMe or $\mathrm{Me}(\mathrm{OMP})), 37.9,37.7\left(\mathrm{CH}_{2}(\mathrm{Lev})\right), 29.9\left(\mathrm{CH}_{3}(\mathrm{Lev})\right), 28.0,27.7,27.6$

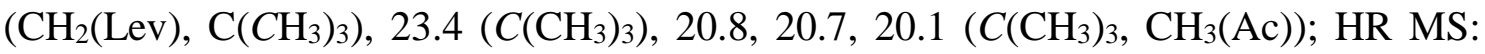
m/z: calcd for $\mathrm{C}_{86} \mathrm{H}_{100} \mathrm{~F}_{6} \mathrm{~N}_{2} \mathrm{O}_{31} \mathrm{SiNa}$ : 1821.5881 ; found: 1821.5903 [M+Na] $]^{+}$.

4-Methoxyphenyl $O$-(3-O-benzyl-2,4-di- $O$-sulfo- $\alpha$-L-idopyranosyluronic $\quad$ acid)$(1 \rightarrow 3)$ - $O$-(2-acetamido-2-deoxy-4,6-di- $O$-sulfo- $\beta$-D-galactopyranosyl)-( $1 \rightarrow 4)-O$ - $(3$ $O$-benzyl-2- $O$-sulfo- $\beta$-D-glucopyranosyluronic $\quad$ acid)-(1 $\rightarrow 3)$-2-acetamido-2-deoxy- 
4,6-di- $\boldsymbol{O}$-sulfo- $\boldsymbol{\beta}$-D-galactopyranoside (30): An excess of (HF) $)_{\mathrm{n}} \cdot \operatorname{Py}(56 \mu \mathrm{L}, 2.1 \mathrm{mmol})$ was added at $0^{\circ} \mathrm{C}$ under an argon atmosphere to a solution of $\mathbf{2 6}(20 \mathrm{mg}, 0.011 \mathrm{mmol})$ in dry THF $(1.0 \mathrm{~mL})$. After $24 \mathrm{~h}$ at $0^{\circ} \mathrm{C}$, the mixture was diluted with $\mathrm{CH}_{2} \mathrm{Cl}_{2}$ and washed with $\mathrm{H}_{2} \mathrm{O}$ and saturated $\mathrm{NaHCO}_{3}$ solution until neutral $\mathrm{pH}$. The organic layer was dried $\left(\mathrm{MgSO}_{4}\right)$, filtered and concentrated in vacuo to give 27. TLC (toluene/EtOAc 1:2) $\mathrm{R}_{\mathrm{f}}$ 0.14; $[\alpha]_{\mathrm{D}}^{20}-4^{\mathrm{o}}\left(c 0.9, \mathrm{CHCl}_{3}\right) ;{ }^{1} \mathrm{H}-\mathrm{NMR}\left(400 \mathrm{MHz}, \mathrm{CDCl}_{3}\right): \delta 7.85(\mathrm{~d}, 2 \mathrm{H}, \mathrm{Ar}), 7.56(\mathrm{t}$, $1 \mathrm{H}, \mathrm{Ar}), 7.48-7.23(\mathrm{~m}, 12 \mathrm{H}, \mathrm{Ar}), 7.13\left(\mathrm{~d}, 1 \mathrm{H}, J_{2, \mathrm{NH}}=8.2 \mathrm{~Hz}, \mathrm{NH}\right), 7.06(\mathrm{~m}, 5 \mathrm{H}, \mathrm{Ar})$, $7.00\left(\mathrm{~d}, 1 \mathrm{H}, J_{2, \mathrm{NH}}=6.4 \mathrm{~Hz}, \mathrm{NH}\right), 6.86(\mathrm{~m}, 2 \mathrm{H}, \mathrm{Ar}), 6.74(\mathrm{~m}, 2 \mathrm{H}, \mathrm{Ar}), 5.36\left(\mathrm{~d}, 1 \mathrm{H}, J_{1,2}=\right.$ $8.4 \mathrm{~Hz}, \mathrm{H}-1 \mathrm{~A}), 5.25$ (q, 2H, $\left.\mathrm{CH}_{2}(\mathrm{Bn})\right), 5.23$ (m, 2H, H-2B, H-4D), 5.16 (bs, 1H, H-4C), 4.98 (bs, 1H, H-1D), 4.93 (bs, 1H, H-5D), 4.80 (m, 3H, H-1B, H-2D, $\mathrm{CH}_{2}(\mathrm{Bn})$ ), 4.63 (q, 2H, $\left.\mathrm{CH}_{2}(\mathrm{Bn})\right), 4.54$ (m, 2H, H-1C, $\left.\mathrm{CH}_{2}(\mathrm{Bn})\right), 4.46$ (bd, 1H, $\left.J_{2,3}=10.6 \mathrm{~Hz}, \mathrm{H}-3 \mathrm{~A}\right), 4.20$ $\left(\mathrm{t}, 1 \mathrm{H}, J_{3,4}=J_{4,5}=8.2 \mathrm{~Hz}, \mathrm{H}-4 \mathrm{~B}\right), 4.13(\mathrm{bs}, 1 \mathrm{H}, \mathrm{H}-4 \mathrm{~A}), 4.08\left(\mathrm{bd}, 1 \mathrm{H}, J_{4,5}=8.8 \mathrm{~Hz}, \mathrm{H}-\right.$ 5B), $4.02(\mathrm{~m}, 1 \mathrm{H}, \mathrm{H}-2 \mathrm{C}), 3.89$ (m, 1H, H-6aC), 3.83-3.71 (m, 12H, H-2A, H-6aA, H-6bA, H-3B, H-3C, H-3D, Me(OMP), COOMe), 3.63 (m, 2H, H-6bC, H-5C), 3.54 (bt, 1H, H-5A), 2.82-2.49 (m, 8H, $\left.\mathrm{CH}_{2}(\mathrm{Lev})\right), 2.18$ (s, 6H, $\left.\mathrm{CH}_{3}(\mathrm{Lev})\right), 1.90,1.64$ (2s, 6H, $\left.\mathrm{CH}_{3}(\mathrm{Ac})\right) ;{ }^{13} \mathrm{C}-\mathrm{NMR}\left(100 \mathrm{MHz}, \mathrm{CDCl}_{3}\right): \delta$ 207.1, 206.4 (CO(Lev)), 171.7, 171.5, 170.5, 170.3, 168.9, 168.8, 165.2 (CO(Lev), CO(Ac), CO(Bz), COOBn, COOMe), $158.0\left(\mathrm{bq},{ }^{2} J_{\mathrm{C}, \mathrm{F}}=37.4 \mathrm{~Hz}, 2 \mathrm{x} C \mathrm{OCF}_{3}\right), 155.8,151.1,137.8,137.6,134.8$ (Ar-C), 133.6, $129.9,129.5,129.3,129.2,128.9,128.5,128.2,128.1,127.9,127.8,127.7,119.0$ (Ar-C, $\operatorname{Ar}-\mathrm{CH}), 116.0\left(\mathrm{q},{ }^{1} J_{\mathrm{C}, \mathrm{F}}=287.6 \mathrm{~Hz}, \mathrm{COCF}_{3}\right), 115.2\left(\mathrm{q},{ }^{1} J_{\mathrm{C}, \mathrm{F}}=287.6 \mathrm{~Hz}, \mathrm{COCF}_{3}\right), 114.7$ (Ar-CH), 101.6 (C-1B), 100.5 (C-1D), 100.1 (C-1C), 98.7 (C-1A), 80.0 (C-3B), 78.5 (C-3A), 77.8 (C-4B), 76.5 (C-3C), $75.1\left(\mathrm{CH}_{2}(\mathrm{Bn})\right), 74.7$ (C-5C), 74.3 (C-5B), 73.3 (C3D), $72.9\left(\mathrm{CH}_{2}(\mathrm{Bn})\right), 72.6(\mathrm{C}-2 \mathrm{~B}), 71.3(\mathrm{C}-5 \mathrm{~A}), 69.4(\mathrm{C}-4 \mathrm{D}), 68.7\left(\mathrm{CH}_{2}(\mathrm{Bn})\right), 68.6$ (C-4A), 68.1 (C-4C), 68.0 (C-2D), 67.3 (C-5D), 62.8 (C-6C), 61.4 (C-6A), 55.8 (COOMe or Me(OMP)), 54.7 (C-2A), 53.9 (C-2C), 52.8 (COOMe or Me(OMP)), 37.9, 
$37.8\left(\mathrm{CH}_{2}(\mathrm{Lev})\right), 29.9,29.8\left(\mathrm{CH}_{3}(\mathrm{Lev})\right), 28.0,27.7\left(\mathrm{CH}_{2}(\mathrm{Lev})\right), 20.6,20.2\left(\mathrm{CH}_{3}(\mathrm{Ac})\right)$;

HR MS: $m / z$ : calcd for $\mathrm{C}_{78} \mathrm{H}_{84} \mathrm{~F}_{6} \mathrm{~N}_{2} \mathrm{O}_{31} \mathrm{Na}$ : 1681.4860 ; found: $1681.4868[M+\mathrm{Na}]^{+}$.

$\mathrm{H}_{2} \mathrm{O}_{2}(30 \%, 0.44 \mathrm{~mL})$ and an aqueous solution of $\mathrm{LiOH}(0.7 \mathrm{M}, 0.27 \mathrm{~mL})$ were added at $-5^{\circ} \mathrm{C}$ to a solution of $27(11.1 \mu \mathrm{mol})$ in THF $(1.5 \mathrm{~mL})$. After stirring for $20 \mathrm{~h}$ at room temperature, $\mathrm{MeOH}(1.5 \mathrm{~mL})$ and an aqueous solution of $\mathrm{NaOH}(4 \mathrm{M}, 0.28 \mathrm{~mL})$ were added. After stirring for $6 \mathrm{~d}$ at room temperature, the reaction mixture was neutralized with Amberlite IR-120 $\left(\mathrm{H}^{+}\right)$resin, filtered, and concentrated to give 28. ESI MS: $m / z$ : calcd for $\mathrm{C}_{45} \mathrm{H}_{57} \mathrm{~N}_{2} \mathrm{O}_{22}$ : 977.3; found: $977.2[M+\mathrm{H}]^{-}$

Triethylamine $(0.4 \mathrm{~mL}$ of a $0.36 \mathrm{M}$ solution in dry $\mathrm{MeOH})$ and acetic anhydride $(21 \mu \mathrm{L}$, $0.22 \mathrm{mmol})$ were added to a cooled $\left(0^{\circ} \mathrm{C}\right)$ solution of $28(11.1 \mu \mathrm{mol})$ in dry $\mathrm{MeOH}(2.5$ $\mathrm{mL})$. After stirring for $2 \mathrm{~h}$ at room temperature, triethylamine $(0.3 \mathrm{~mL})$ was added and the mixture was concentrated to dryness. The residue was purified by Sephadex LH-20 chromatography $\left(\mathrm{CH}_{2} \mathrm{Cl}_{2}-\mathrm{MeOH}\right.$ 1:1) to give 29 as triethylammonium salt. The sodium salt of 29 was obtained by treatment with Amberlite IR-120 $\mathrm{H}^{+}$resin in $\mathrm{MeOH}(\mathrm{pH} \sim$ 3), followed by filtration, treatment with $0.1 \mathrm{M} \mathrm{NaOH}(\mathrm{pH} \sim 7)$ and concentration.

${ }^{1} \mathrm{H}-\mathrm{NMR}(500 \mathrm{MHz}, \mathrm{MeOD}$, data for sodium salt): $\delta 7.58$ (d, 2H, Ar), 7.45 (d, 2H, Ar), 7.34-7.21 (m, 6H, Ar), 6.98 (m, 2H, Ar), 6.82 (m, 2H, Ar), 5.06 (d, 1H, $\left.\mathrm{CH}_{2}(\mathrm{Bn})\right), 4.93$ $\left(\mathrm{d}, 1 \mathrm{H}, J_{1,2}=8.4 \mathrm{~Hz}, \mathrm{H}-1 \mathrm{~A}\right), 4.89\left(\mathrm{~d}, 1 \mathrm{H}, J_{1,2}=4.4 \mathrm{~Hz}, \mathrm{H}-1 \mathrm{D}\right), 4.77$ (q, 2H, $\left.\mathrm{CH}_{2}(\mathrm{Bn})\right)$, $4.73\left(\mathrm{~d}, 1 \mathrm{H}, \mathrm{CH}_{2}(\mathrm{Bn})\right), 4.62\left(\mathrm{~d}, 1 \mathrm{H}, J_{1,2}=8.5 \mathrm{~Hz}, \mathrm{H}-1 \mathrm{C}\right), 4.43$ (m, 1H, H-1B), 4.41 (d, $\left.1 \mathrm{H}, J_{4,5}=3.7 \mathrm{~Hz}, \mathrm{H}-5 \mathrm{D}\right), 4.27\left(\mathrm{dd}, 1 \mathrm{H}, J_{2,3}=10.6 \mathrm{~Hz}, \mathrm{H}-2 \mathrm{~A}\right), 4.23\left(\mathrm{dd}, 1 \mathrm{H}, J_{2,3}=10.4\right.$ Hz, H-2C), 4.13 (d, 1H, J3,4 = 2.7 Hz, H-4A), 4.05 (d, 1H, J J 3.95 (m, 2H, H-4B, H-4D), 3.84-3.69 (m, 9H, H-6aA, H-6aC, H-6bA or H-6bC, H-3A (3.80), Me(OMP) (3.74), H-5B (3.72), H-3C (3.71)), 3.65-3.58 (m, 3H, H-6bA or H-6bC, H-2D, H-5A or H-5C), 3.55 (t, $\left.1 \mathrm{H}, J_{2,3}=J_{3,4}=5.9 \mathrm{~Hz}, \mathrm{H}-3 \mathrm{D}\right), 3.52(\mathrm{~m}, 1 \mathrm{H}, \mathrm{H}-$ 5A or H-5C), 3.47-3.44 (m, 2H, H-2B, H-3B), 2.05, 1.99 (2s, 6H, NHAc); ${ }^{13} \mathrm{C}-\mathrm{NMR}$ 
(125 MHz, MeOD) (selected data from HSQC experiment): $\delta$ 129.9-128.4, 118.9, 115.1 (Ar-CH), 105.6 (C-1B), 103.9 (C-1D), 102.1 (C-1A), 101.3 (C-1C), 83.7 (C-3B), 81.8 (C-3C), 81.7 (C-3A), 80.3 (C-3D), 78.9 (C-4B), 77.7 (C-5B), 77.2, 76.5 (C-5A, C-5C), $76.4\left(\mathrm{CH}_{2}(\mathrm{Bn})\right), 74.0(\mathrm{C}-2 \mathrm{~B}), 73.6\left(\mathrm{CH}_{2}(\mathrm{Bn})\right), 71.7$ (C-5D), $71.2(\mathrm{C}-4 \mathrm{D}), 70.6(\mathrm{C}-2 \mathrm{D})$, 69.4 (C-4C), 68.8 (C-4A), 62.7, 62.3 (C-6A, C-6C), 55.7 (Me(OMP)), 52.7 (C-2C), 52.6 (C-2A), 23.1, 22.8 (NAc); ESI MS: $m / z$ : calcd for $\mathrm{C}_{49} \mathrm{H}_{61} \mathrm{~N}_{2} \mathrm{O}_{24}$ : 1061.4; found: $1061.2[M+\mathrm{H}]^{-}$.

Compound $29(11.1 \mu \mathrm{mol})$ and sulfur trioxide-trimethylamine complex (54 mg, 0.39 mmol) were dissolved in dry $\operatorname{DMF}(1.5 \mathrm{~mL})$ and heated at $100^{\circ} \mathrm{C}$ for $2 \mathrm{~h} \mathrm{using}$ microwave radiation ( $20 \mathrm{~W}$ average power). The reaction vessel was cooled and $\mathrm{Et}_{3} \mathrm{~N}$ $(150 \mu \mathrm{L})$ and $\mathrm{MeOH}(1 \mathrm{~mL})$ were added. The solution was layered on the top of a Sephadex LH-20 chromatography column which was eluted with $\mathrm{MeOH}$ to obtain $\mathbf{3 0}$ as triethylammonium salt. The residue was converted into the sodium salt by elution from a column of Dowex 50WX4-Na ${ }^{+}$with $\mathrm{MeOH}-\mathrm{H}_{2} \mathrm{O}$ 9:1 (6.9 mg, 34\% from 26; 4 steps, $76 \%$ average yield per step). Due to extensive overlap of ${ }^{1} \mathrm{H}-\mathrm{NMR}$ signals of the sodium salt at $25^{\circ} \mathrm{C}, \mathbf{3 0}$ was characterized as calcium salt at $40^{\circ} \mathrm{C}$. The calcium salt of $\mathbf{3 0}$ was obtained by adding a $0.9 \mathrm{M}$ solution of $\mathrm{CaCl}_{2}$ in $\mathrm{D}_{2} \mathrm{O} .{ }^{1} \mathrm{H}-\mathrm{NMR}\left(500 \mathrm{MHz}, \mathrm{D}_{2} \mathrm{O}, 40^{\circ} \mathrm{C}\right.$, data for calcium salt): $\delta 7.73(\mathrm{~d}, 2 \mathrm{H}, \mathrm{Ar}), 7.63-7.46(\mathrm{~m}, 8 \mathrm{H}, \mathrm{Ar}), 7.23(\mathrm{~m}, 2 \mathrm{H}, \mathrm{Ar}), 7.11$ (m, 2H, Ar), 5.44 (bs, 1H, H-1D), 5.37 (d, 1H, $\left.J_{1,2}=8.6 \mathrm{~Hz}, \mathrm{H}-1 \mathrm{~A}\right), 5.30$ (bs, $1 \mathrm{H}$, H-5D), 5.11 (bd, 1H, H-4A), 5.02 (bs, 1H, H-4C), 4.98 (q, 2H, $\left.\mathrm{CH}_{2}(\mathrm{Bn})\right), 4.91-4.81$ (m, 5H, H-1B (4.90), H-4D (4.89), $\left.\mathrm{CH}_{2}(\mathrm{Bn}), \mathrm{H}-1 \mathrm{C}(4.84)\right), 4.52$ (t, $1 \mathrm{H}, J_{1,2}=J_{2,3}=7.5 \mathrm{~Hz}$, H-2B), $4.47\left(\mathrm{dd}, 1 \mathrm{H}, J_{5,6 \mathrm{a}}=2.6 \mathrm{~Hz}, J_{6 \mathrm{a}, 6 \mathrm{~b}}=11.3 \mathrm{~Hz}, \mathrm{H}-6 \mathrm{aA}\right.$ or H-6aC), 4.43-4.19 (m, 10H, H-2D (4.43), H-6aA or H-6aC, H-3A (4.39), H-3D (4.36), H-6bA, H-6bC, H-5A or H-5C (4.33), H-4B (4.29), H-2C (4.24), H-2A (4.22)), 4.14 (m, 1H, H-3C), 4.10 (bt, 1H, H-5A or H-5C), 3.99-3.94 (m, 5H, H-3B (3.98), H-5B (3.97), Me(OMP) (3.94)), 
2.21 (s, 6H, NHAc); ${ }^{13} \mathrm{C}-\mathrm{NMR}\left(125 \mathrm{MHz}, \mathrm{D}_{2} \mathrm{O}, 40^{\circ} \mathrm{C}\right.$ ) (selected data from HSQC experiment): $\delta$ 129.6-128.5, 118.6, 115.2 (Ar-CH), 102.2 (C-1B), 101.5 (C-1D), 100.6 (C-1A), 100.4 (C-1C), 80.2 (C-3B), 79.6 (C-3C), 79.1 (C-2B), 77.5 (C-4B), 77.4 (C5B), 76.7 (C-3A), 75.7 (C-4A), 75.3 (C-4C), $74.2\left(\mathrm{CH}_{2}(\mathrm{Bn})\right), 72.8$ (C-5A or C-5C), 72.6 (C-4D), 72.4 (C-5A or C-5C), 71.9 (C-3D), $71.4\left(\mathrm{CH}_{2}(\mathrm{Bn})\right), 71.2(\mathrm{C}-2 \mathrm{D}), 68.2(\mathrm{C}-$ 5D), 67.8, 67.4 (C-6A, C-6C), 56.0 (Me(OMP)), 52.4 (C-2A), 52.2 (C-2C), 22.8 (NAc); ESI MS: $m / z$ : calcd for $\mathrm{C}_{49} \mathrm{H}_{55} \mathrm{~N}_{2} \mathrm{O}_{45} \mathrm{~S}_{7} \mathrm{Na}$ : 865.0 ; found: $865.0[M+5 \mathrm{Na}+2 \mathrm{H}]^{2-} ; \mathrm{HR}$ MS: $m / z$ : calcd for $\mathrm{C}_{49} \mathrm{H}_{60} \mathrm{~N}_{2} \mathrm{O}_{45} \mathrm{~S}_{7}: 810.0262$; found: $810.0253[M+7 \mathrm{H}]^{2-}$.

4-Methoxyphenyl $O$-(2,4-di- $O$-sulfo- $\alpha$-L-idopyranosyluronic $\quad$ acid)-(1 $\rightarrow 3$ )- $O$-(2acetamido-2-deoxy-4,6-di- $O$-sulfo- $\beta$-D-galactopyranosyl)-(1 $\rightarrow 4)-O$ - $(2-O$-sulfo- $\beta$-Dglucopyranosyluronic $\quad$ acid $)-(1 \rightarrow 3)$-2-acetamido-2-deoxy-4,6-di- $O$-sulfo- $\beta$-Dgalactopyranoside (1): A solution of $\mathbf{3 0}$ (4.9 mg, $2.7 \mu$ mol, sodium salt) in $\mathrm{H}_{2} \mathrm{O} / \mathrm{MeOH}$ (4.5 mL/0.5 mL) was hydrogenated $(1.5 \mathrm{~atm})$ in the presence of $\mathrm{Pd}(\mathrm{OH})_{2}$. After $22 \mathrm{~h}$, the suspension was filtered over Celite and concentrated. The residue was purified by Sephadex G-25 chromatography $\left(\mathrm{H}_{2} \mathrm{O} / \mathrm{MeOH}\right.$ 9:1) to give $\mathbf{1}$ as sodium salt after lyophilisation (3.6 mg, 82\%; $28 \%$ from 26, 5 steps, $78 \%$ average yield per step). ${ }^{1} \mathrm{H}-\mathrm{NMR}\left(500 \mathrm{MHz}, \mathrm{D}_{2} \mathrm{O}\right): \delta 7.10$ (d, 2H, Ar), 6.98 (d, 2H, Ar), 5.29 (bs, 1H, H-1D), $5.22\left(\mathrm{~d}, 1 \mathrm{H}, J_{1,2}=8.4 \mathrm{~Hz}, \mathrm{H}-1 \mathrm{~A}\right), 4.98$ (bs, 1H, H-5D), 4.95 (bs, 1H, H-4A), 4.76 (m, 1H, H-4C), 4.72 (m, 1H, H-1C), 4.70 (d, 1H, $\left.J_{1,2}=7.7 \mathrm{~Hz}, \mathrm{H}-1 \mathrm{~B}\right), 4.66$ (bs, 1H, H-4D), 4.43 (m, 1H, H-3D), 4.35 (bd, 1H, H-6aA or H-6aC), 4.31-4.20 (m, 7H, H-6aA or H6aC, H-3A (4.27), H-6bA, H-6bC, H-5A or H-5C (4.23), H-2B (4.22), H-2D (4.20)), 4.14-4.07 (m, 4H, H-2A, H-2C, H-5A or H-5C, H-3C), $3.93\left(\mathrm{t}, 1 \mathrm{H}, J_{3,4}=J_{4,5}=9.1 \mathrm{~Hz}\right.$, H-4B), 3.82-3.81 (m, 4H, Me(OMP), H-3B), 3.73 (d, 1H, $\left.J_{4,5}=9.4 \mathrm{~Hz}, \mathrm{H}-5 \mathrm{~B}\right), 2.11$, 2.05 (2s, 6H, NHAc); ${ }^{13} \mathrm{C}-\mathrm{NMR}\left(125 \mathrm{MHz}, \mathrm{D}_{2} \mathrm{O}\right)$ (selected data from HSQC experiment): $\delta$ 118.8, 115.5 (Ar-CH), 103.1 (C-1B), 101.3 (C-1C), 101.0 (C-1A), 100.7 
(C-1D), 81.0 (C-4B), 80.1 (C-2B), 77.0 (C-5B, C-3A), 76.6 (C-3C), 76.4 (C-4A), 76.1

(C-4C), 74.8 (C-4D), 73.9 (C-3B), 73.2, 72.7 (C-5A, C-5C), 72.6 (C-2D), 68.5, 67.9 (C-

6A, C-6C), 67.0 (C-5D), 66.5 (C-3D), 56.2 (Me(OMP)), 52.8, 52.4 (C-2A, C-2C), 23.2,

22.8 (NAc); ESI MS: $m / z$ : calcd for $\mathrm{C}_{35} \mathrm{H}_{43} \mathrm{~N}_{2} \mathrm{O}_{45} \mathrm{~S}_{7} \mathrm{Na}_{5}$ : 774.9; found: 774.8 $[M+5 \mathrm{Na}+2 \mathrm{H}]^{2-} ;$ HR MS: $m / z$ : calcd for $\mathrm{C}_{35} \mathrm{H}_{48} \mathrm{~N}_{2} \mathrm{O}_{45} \mathrm{~S}_{7}$ : 719.9792; found: 719.9786 $[M+7 \mathrm{H}]^{2-}$.

\section{Fluorescence polarization assays}

Fluorescence polarization measurements were performed in 384-well microplates (black polystyrene, non-treated, Corning) using a TRIAD multimode reader (Dynex).

Fluorescent probes 36-40, recombinant human FGF-2 (Peprotech), and inhibitors were dissolved in PBS buffer (10 mM, pH 7.4). For direct binding, $20 \mu \mathrm{L}$ of a fluorescent probe solution ( $20 \mathrm{nM}$ ) were transferred to each well. Then $20 \mu \mathrm{L}$ of FGF-2 solution (concentration ranging from $1.45 \mu \mathrm{M}$ to $23 \mathrm{nM}$ ) were added and the microplate was shaked in the dark for $5 \mathrm{~min}$, before reading. The total sample volume in each well was $40 \mu \mathrm{L}$. Control wells contained $20 \mu \mathrm{L}$ of the fluorescent probe solution and $20 \mu \mathrm{L}$ of PBS buffer. Blank wells contained $20 \mu \mathrm{L}$ of FGF-2 solution and $20 \mu \mathrm{L}$ of PBS buffer and their measurements were substracted from all values. All samples were performed in replicates of three. For inhibition assay, $10 \mu \mathrm{L}$ of probe and $20 \mu \mathrm{L}$ of protein at fixed concentration (40 nM and $205 \mathrm{nM}$, respectively) were mixed with $10 \mu \mathrm{L}$ of inhibitor solution $(100 \mu \mathrm{M})$. The total sample volume in each well was again $40 \mu \mathrm{L}$. After stirring for $5 \mathrm{~min}$ in the dark, fluorescence polarization was recorded. Two control wells containing no inhibitor and probe only (no FGF-2) were included in the study. For the determination of $\mathrm{IC}_{50}$ value, wells containing probe and FGF-2 at fixed concentration, as described above, were incubated with 6 different concentrations of inhibitor, ranging from $0.025 \mu \mathrm{M}$ to $100 \mu \mathrm{M}$. Average polarization values of six replicate wells were 
plotted against the logarithm of inhibitor concentration, and the curve was fitted to the simplified formula corresponding to a one-site competitive interaction. All the experiments were repeated at least twice.

Occasionally, we found loss of FGF-2 activity when working with low concentrated aliquots in PBS buffer. For this reason, all measurements were alternatively done in PBS $+0.5 \%$ BSA (data not shown), getting similar results than those obtained with PBS.

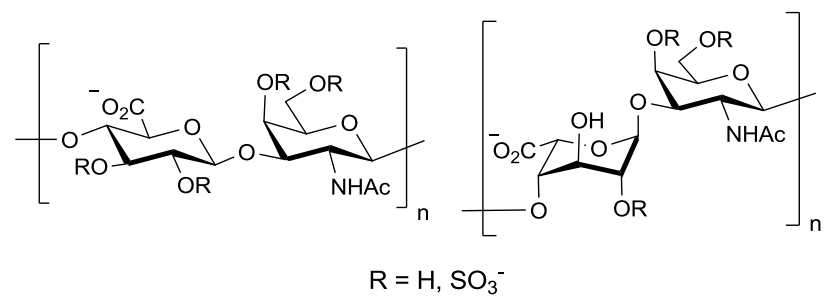

Figure 1. Disaccharide repeating units of chondroitin (left) and dermatan (right) sulfate with potential sites of sulfation indicated.

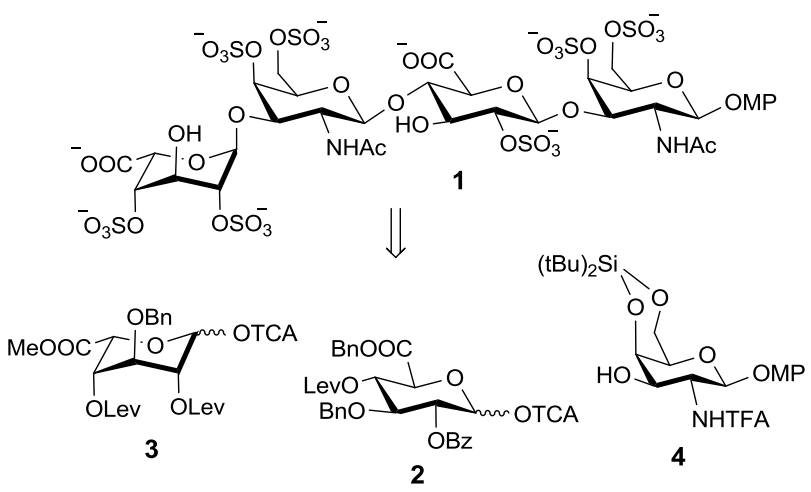

Scheme 1. Building blocks required for the synthesis of tetrasaccharide $\mathbf{1}$. 


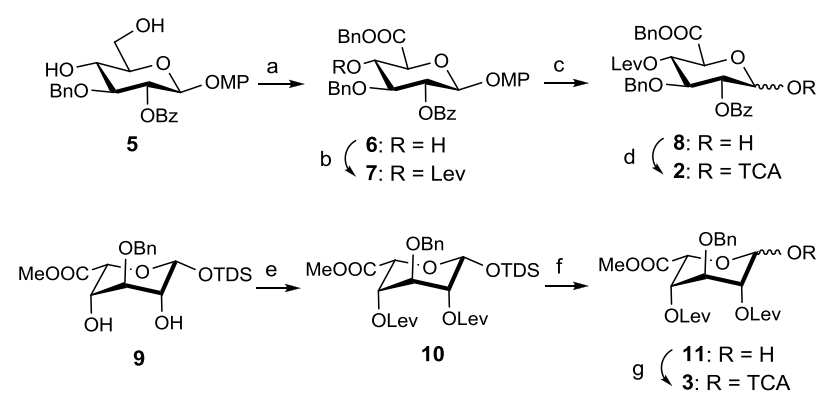

Scheme 2. a) TEMPO, $\mathrm{Bu}_{4} \mathrm{NBr}, \mathrm{KBr}, \mathrm{Ca}(\mathrm{ClO})_{2}, \mathrm{NaHCO}_{3}, \mathrm{CH}_{2} \mathrm{Cl}_{2} / \mathrm{H}_{2} \mathrm{O}, 0^{\circ} \mathrm{C} ; \mathrm{BnBr}$, DMF, Bu $4 \mathrm{NI}, 60^{\circ} \mathrm{C}, 56 \%$; b) LevOH, DCC, DMAP, $\mathrm{CH}_{2} \mathrm{Cl}_{2}, 81 \%$; c) CAN, toluene/ $\mathrm{CH}_{3} \mathrm{CN} / \mathrm{H}_{2} \mathrm{O}, 60 \%$; d) $\mathrm{Cl}_{3} \mathrm{CCN}, \mathrm{K}_{2} \mathrm{CO}_{3}, \mathrm{CH}_{2} \mathrm{Cl}_{2}, 96 \%$; e) $\mathrm{Lev}_{2} \mathrm{O}, \mathrm{Py} / \mathrm{CH}_{2} \mathrm{Cl}_{2}$, DMAP, 98\%; f) (HF)n . Py, THF, $0^{\circ} \mathrm{C}, 77 \%$; g) $\mathrm{Cl}_{3} \mathrm{CCN}, \mathrm{K}_{2} \mathrm{CO}_{3}, \mathrm{CH}_{2} \mathrm{Cl}_{2}, 95 \%$.

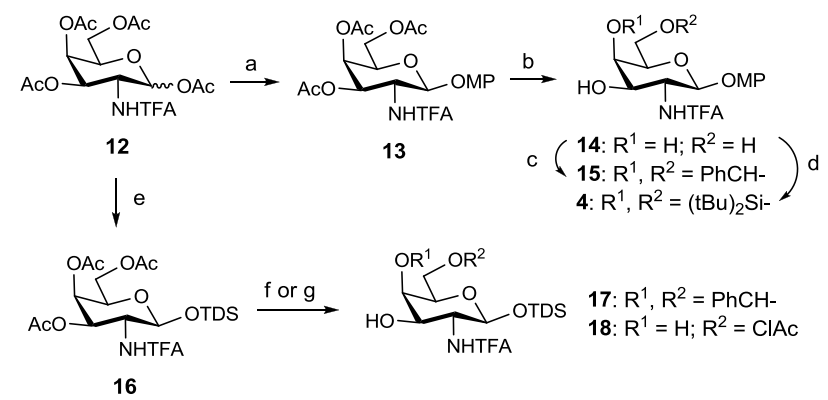

Scheme 3. a) 4-methoxyphenol, TMSOTf, $\mathrm{CH}_{2} \mathrm{Cl}_{2}, 0^{\circ} \mathrm{C}, 42 \%+40 \%$ starting material; b) $\mathrm{NaOMe}, \mathrm{MeOH}$, quantitative; c) $\mathrm{PhCH}(\mathrm{OMe})_{2}, p-\mathrm{TsOH}, \mathrm{CH}_{3} \mathrm{CN}, 87 \%$; d) tBu $2 \mathrm{Si}(\mathrm{OTf})_{2}, \mathrm{Py}, 89 \%$; e) $\mathrm{BnNH}_{2}$, THF; TDSCl, imidazole, $\mathrm{CH}_{2} \mathrm{Cl}_{2}, 51 \%$; f) $\mathrm{NaOMe}$, $\mathrm{MeOH} ; \mathrm{PhCH}(\mathrm{OMe})_{2}, p$-TsOH, $\left.\mathrm{CH}_{3} \mathrm{CN} / \mathrm{DMF} ; \mathrm{g}\right) \mathrm{NaOMe}, \mathrm{MeOH} ;(\mathrm{ClAc})_{2} \mathrm{O}$, collidine, $\mathrm{CH}_{2} \mathrm{Cl}_{2},-60^{\circ} \mathrm{C}, 77 \%$. 


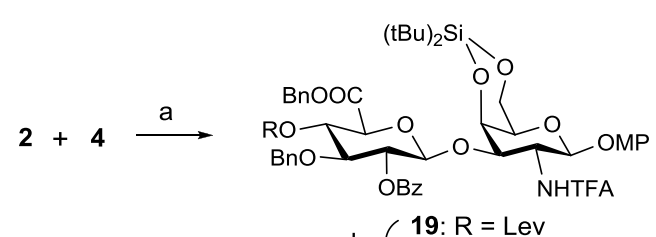

$b\left(\begin{array}{l}19: R=L e v \\ 25: R=H\end{array}\right.$

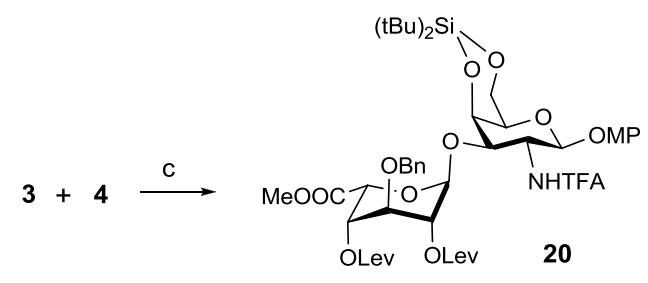

Scheme 4. a) TMSOTf, $\mathrm{CH}_{2} \mathrm{Cl}_{2}, 0^{\circ} \mathrm{C}, 91 \%$; b) $\mathrm{NH}_{2} \mathrm{NH}_{2} \cdot \mathrm{H}_{2} \mathrm{O}, \mathrm{Py} / \mathrm{AcOH}, \mathrm{CH}_{2} \mathrm{Cl}_{2}, 84 \%$;

c) TMSOTf, $\mathrm{CH}_{2} \mathrm{Cl}_{2}, 0^{\circ} \mathrm{C}, 79 \%$.

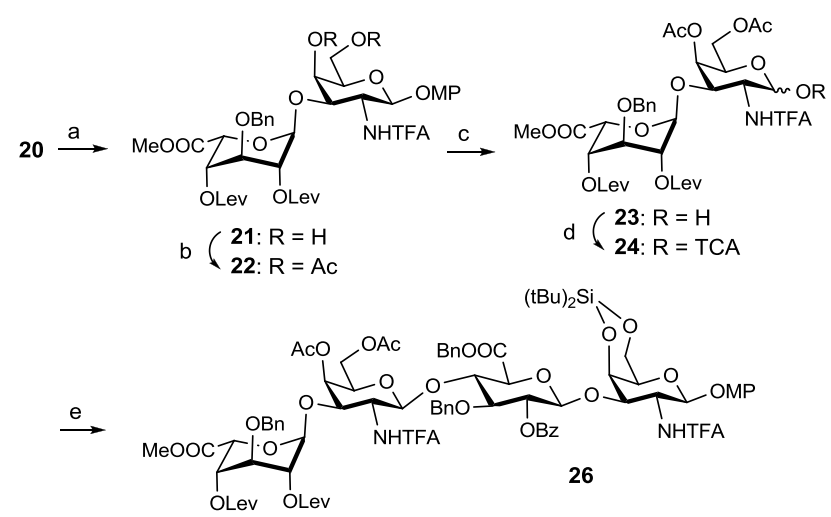

Scheme 5. a) (HF) $)_{n} \cdot \mathrm{Py}, \mathrm{THF}, 0^{\circ} \mathrm{C}, 97 \%$; b) $\mathrm{Ac}_{2} \mathrm{O}, \mathrm{Py}, \mathrm{DMAP}, 89 \%$; c) CAN,

toluene/ $\mathrm{CH}_{3} \mathrm{CN} / \mathrm{H}_{2} \mathrm{O}, 0^{\circ} \mathrm{C}, 76 \%$; d) $\mathrm{Cl}_{3} \mathrm{CCN}, \mathrm{DBU}, \mathrm{CH}_{2} \mathrm{Cl}_{2}, 84 \%$; e) 25, TMSOTf,

$\mathrm{CH}_{2} \mathrm{Cl}_{2}, 0^{\circ} \mathrm{C}, 74 \%$. 


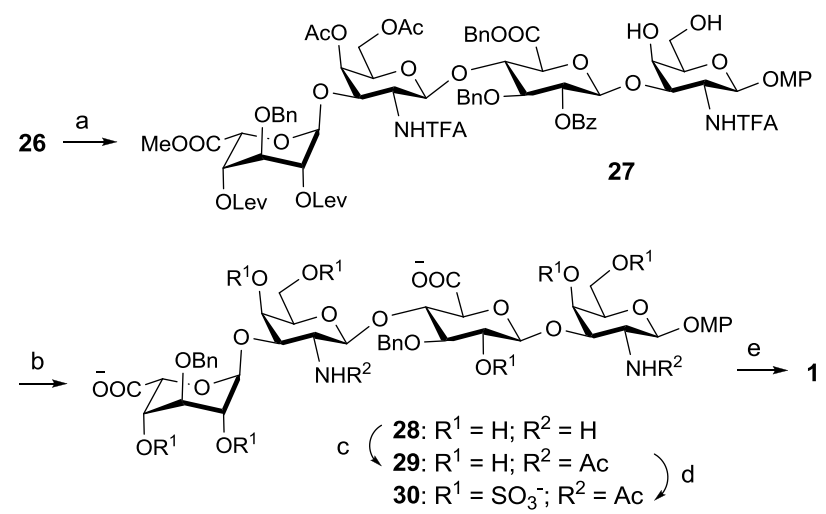

Scheme 6. a) (HF) $)_{n} \cdot \mathrm{Py}, \mathrm{THF}, 0^{\circ} \mathrm{C}$; b) $\mathrm{LiOH}, \mathrm{H}_{2} \mathrm{O}_{2}$, THF; $\mathrm{NaOH}, \mathrm{MeOH}$; c) $\mathrm{Ac}_{2} \mathrm{O}$, $\mathrm{MeOH}, \mathrm{Et}_{3} \mathrm{~N}$; d) $\mathrm{SO}_{3} \cdot \mathrm{Me}_{3} \mathrm{~N}, \mathrm{DMF}, 100^{\circ} \mathrm{C}, \mathrm{MW}, 34 \%$ from 26, 4 steps, $76 \%$ average yield per step; e) $\mathrm{H}_{2}, \mathrm{Pd}(\mathrm{OH})_{2}, \mathrm{H}_{2} \mathrm{O} / \mathrm{MeOH}, 82 \%$.

Table 1. ${ }^{1} \mathrm{H}-\mathrm{NMR}$ chemical shifts for sulfated positions of compounds $\mathbf{3 0}$ and $\mathbf{1}$ and the corresponding non-sulfated positions of 29.

\begin{tabular}{cccccccc}
\hline Compound & H-4A & H-6A & H-2B & H-4C & H-6C & H-2D & H-4D \\
\hline $\mathbf{2 9}^{\mathrm{a}}$ & 4.13 & $3.84-3.58$ & $3.47-3.44$ & 4.05 & $3.84-3.58$ & $3.65-3.58$ & $3.99-3.95$ \\
$\mathbf{3 0}^{\mathrm{b}}$ & 5.11 & $4.47-4.19$ & 4.52 & 5.02 & $4.47-4.19$ & 4.43 & 4.89 \\
$\mathbf{1}^{\mathrm{c}}$ & 4.95 & $4.35-4.20$ & 4.22 & 4.76 & $4.35-4.20$ & 4.20 & 4.66 \\
\hline
\end{tabular}

${ }^{\mathrm{a}}$ sodium salt, in MeOD; ${ }^{\mathrm{b}}$ calcium salt, in $\mathrm{D}_{2} \mathrm{O}$; ${ }^{\text {c }}$ sodium salt, in $\mathrm{D}_{2} \mathrm{O}$

Table 2. ${ }^{13} \mathrm{C}$-NMR chemical shifts for sulfated positions of compounds $\mathbf{3 0}$ and $\mathbf{1}$ and the corresponding non-sulfated positions of 29.

\begin{tabular}{cccccccc}
\hline Compound & C-4A & C-6A & C-2B & C-4C & C-6C & C-2D & C-4D \\
\hline $\mathbf{2 9}^{\mathrm{a}}$ & 68.8 & 62.7 or 62.3 & 74.0 & 69.4 & 62.7 or 62.3 & 70.6 & 71.2 \\
$\mathbf{3 0}^{\mathrm{b}}$ & 75.7 & 67.8 or 67.4 & 79.1 & 75.3 & 67.8 or 67.4 & 71.2 & 72.6 \\
$\mathbf{1}^{\mathrm{c}}$ & 76.4 & 68.5 or 67.9 & 80.1 & 76.1 & 68.5 or 67.9 & 72.6 & 74.8 \\
\hline
\end{tabular}

${ }^{\mathrm{a}}$ sodium salt, in MeOD; ${ }^{\mathrm{b}}$ calcium salt, in $\mathrm{D}_{2} \mathrm{O}$; ${ }^{\mathrm{c}}$ sodium salt, in $\mathrm{D}_{2} \mathrm{O}$ 

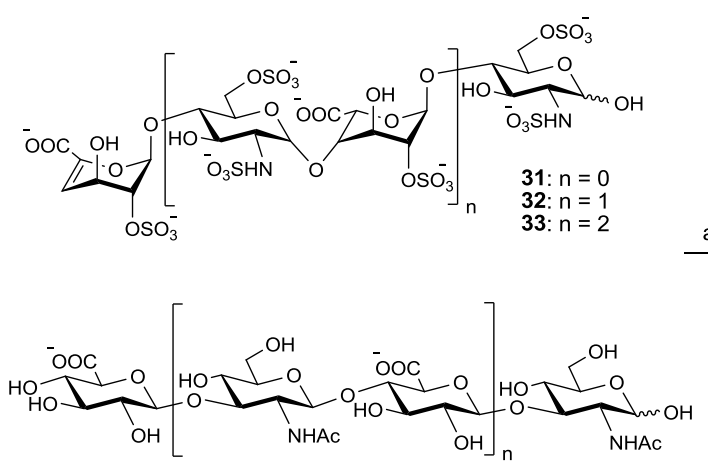

34: $n=1$
35: $n=2$
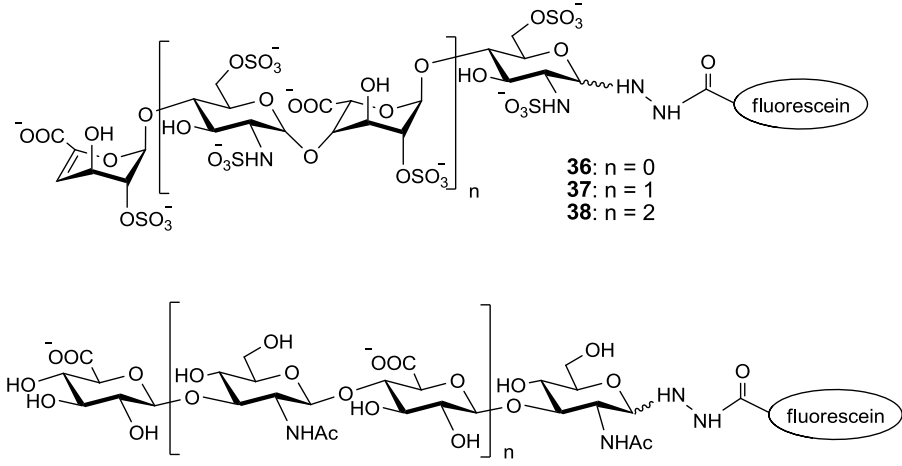

39: $n=1$

Scheme 7. a) Fluorescein hydrazide, DMSO/phosphate buffer pH $5.5(1: 1), 30^{\circ} \mathrm{C}, 91 \%$ (36), $91 \%$ (37), $94 \%$ (38), $92 \%$ (39), $95 \%$ (40).

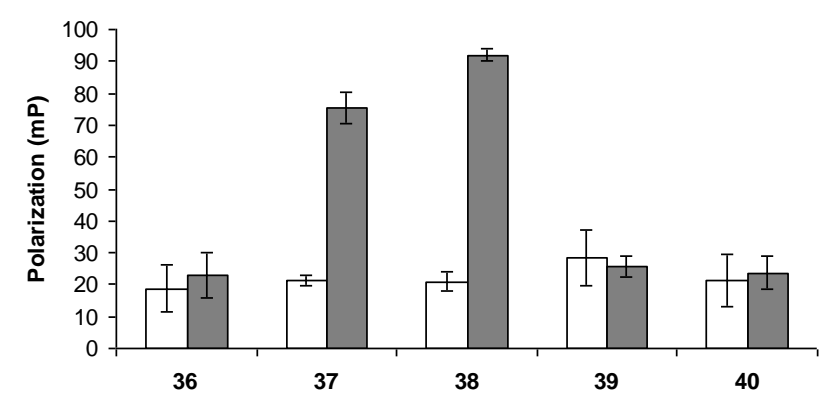

Figure 2. Fluorescence polarization values (right, in grey) from wells containing fluorescent GAG oligosaccharides 36-40 (10 nM) and FGF-2 (97 nM) are compared with the values obtained in the absence of the protein (left, in white). For each oligosaccharide, polarization values are the average of three replicate wells and the error bars show the standard deviations for these measurements. 


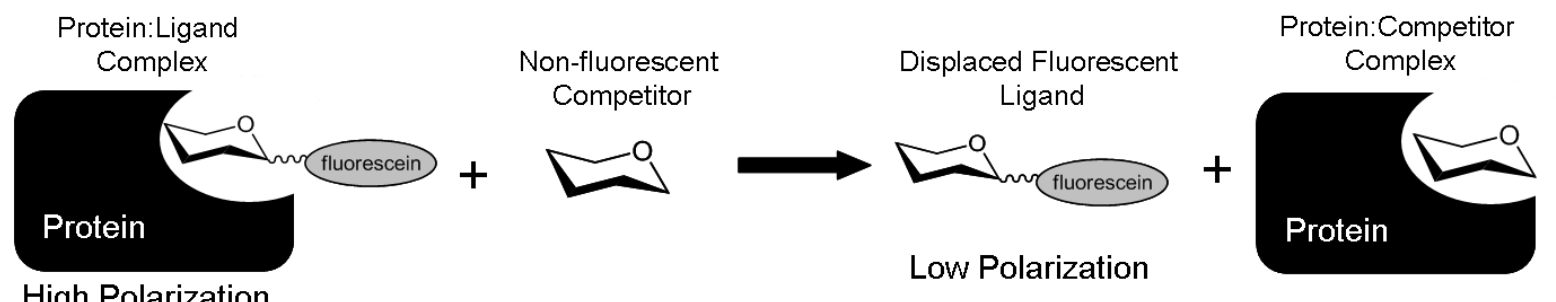

Figure 3. Schematic representation of the competition assay. The displacement of a fluorescent sugar from a protein receptor by an active competitor results in a decrease of the polarization value. Thus, the binding affinities of non-fluorescent compounds can be estimated.
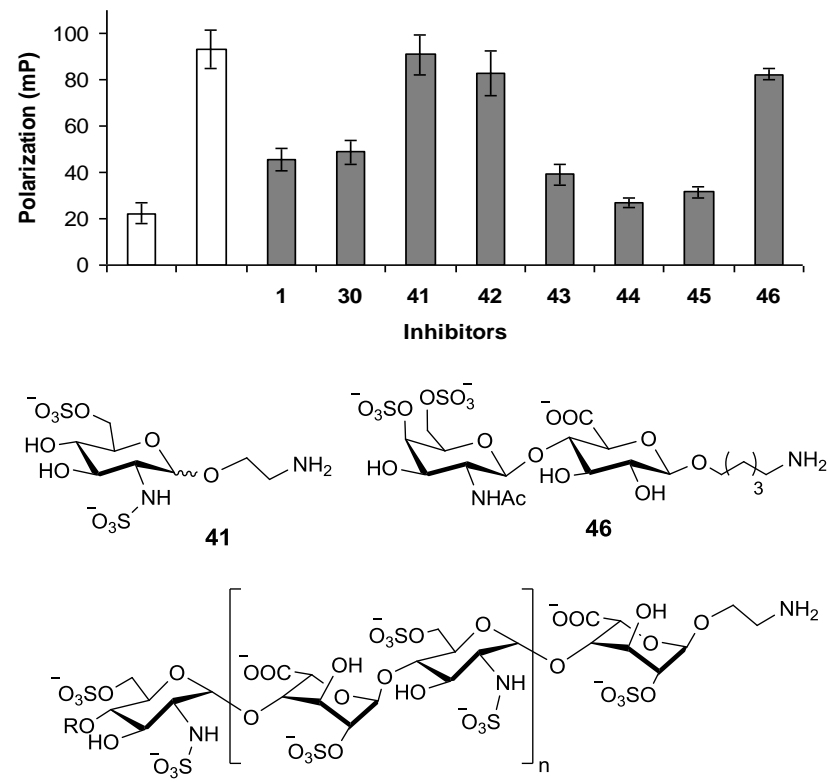

42: $\mathrm{n}=0 ; \mathrm{R}=\mathrm{H} ; \mathbf{4 3}: \mathrm{n}=1 ; \mathrm{R}=\mathrm{H}$;

44: $\mathrm{n}=2 ; \mathrm{R}=\mathrm{H} ; 45: \mathrm{n}=2 ; \mathrm{R}=\mathrm{SO}_{3}$

Figure 4. Competition assay to analyse the inhibitory potency of a collection of synthetic oligosaccharides $(\mathbf{1}, \mathbf{3 0}, \mathbf{4 1}-\mathbf{4 6})$. The graphic presents the polarization values obtained from wells containing $25 \mu \mathrm{M}$ inhibitor, $103 \mathrm{nM}$ FGF-2, and $10 \mathrm{nM}$ fluorescent 38. Control wells (in white) correspond to samples with probe only (left) and no inhibitor (right) and indicate the expected values for $100 \%$ and $0 \%$ inhibition, respectively. All the measurements are the average of three replicate wells and the error bars show the standard deviations for these measurements. 


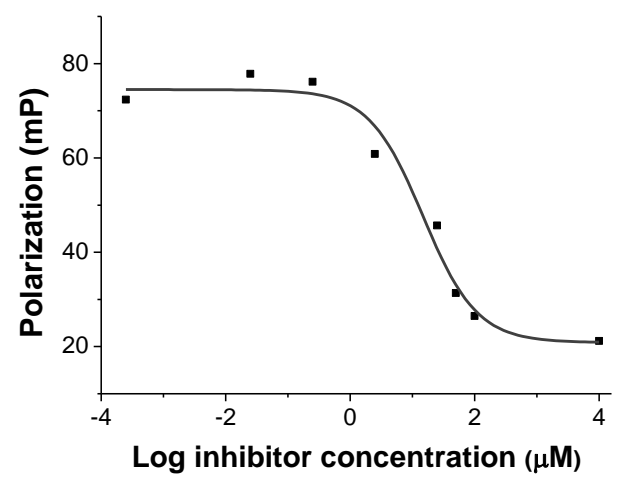

Figure 5. Inhibition curve showing the ability of tetrasacharide 1, at different concentrations (from $0.025 \mu \mathrm{M}$ to $100 \mu \mathrm{M}$ ), to inhibit the interaction between FGF-2 $(103 \mathrm{nM})$ and probe $38(10 \mathrm{nM})$. The concentration required to inhibit 50\% binding ( $\mathrm{IC}_{50}$ value) was calculated from data analysis (see main text). Control wells, with no inhibitor and no protein, were included in the fitting. All the polarization values are the average of six replicate wells.

\section{Acknowledgements}

We thank the Spanish Ministry of Economy and Competitiveness (Grants CTQ200907168 and CTQ2012-32605), the Regional Government of Andalusia (Grant P07-FQM02969), the Spanish National Research Council (CSIC, Grant 201180E021) and the European Union (FEDER support) for financial support. JA acknowledges financial support from the Spanish Ministry of Economy and Competitiveness through the Ramón y Cajal program.

\section{References and notes}

1. K. Sugahara, T. Mikami, T. Uyama, S. Mizuguchi, K. Nomura and H. Kitagawa, Curr. Opin. Struct. Biol., 2003, 13, 612-620.

2. J. M. Trowbridge and R. L. Gallo, Glycobiology, 2002, 12, 117R-125R.

3. C. I. Gama, S. E. Tully, N. Sotogaku, P. M. Clark, M. Rawat, N. Vaidehi, W. A. Goddard, A. Nishi and L. C. Hsieh-Wilson, Nat. Chem. Biol., 2006, 2, 467-473.

4. M. Petitou and C. A. A. van Boeckel, Angew. Chem. Int. Ed., 2004, 43, 31183133. 
5. J. L. de Paz and P. H. Seeberger, Mol. BioSyst., 2008, 4, 707-711.

6. C. I. Gama and L. C. Hsieh-Wilson, Curr. Opin. Chem. Biol., 2005, 9, 609-619.

7. A. K. Powell, E. A. Yates, D. G. Fernig and J. E. Turnbull, Glycobiology, 2004, 14, 17R-30R.

8. I. Capila and R. J. Linhardt, Angew. Chem. Int. Ed., 2002, 41, 391-412.

9. $\quad$ N. Barroca and J. C. Jacquinet, Carbohydr. Res., 2002, 337, 673-689.

10. E. Bedini and M. Parrilli, Carbohydr. Res., 2012, 356, 75-85.

11. P. Bourhis, F. Machetto, P. Duchaussoy, J. P. Herault, J. M. Mallet, J. M. Herbert, M. Petitou and P. Sinay, Bioorg. Med. Chem. Lett., 1997, 7, 2843-2846.

12. F. Goto and T. Ogawa, Bioorg. Med. Chem. Lett., 1994, 4, 619-624.

13. A. Lubineau and D. Bonnaffe, Eur. J. Org. Chem., 1999, 2523-2532.

14. M. Rawat, C. I. Gama, J. B. Matson and L. C. Hsieh-Wilson, J. Am. Chem. Soc., 2008, 130, 2959-2961.

15. S.-G. Lee, J. M. Brown, C. J. Rogers, J. B. Matson, C. Krishnamurthy, M. Rawat and L. C. Hsieh-Wilson, Chem. Sci., 2010, 1, 322-325.

16. G. Despras, C. Bernard, A. Perrot, L. Cattiaux, A. Prochiantz, H. Lortat-Jacob and J.-M. Mallet, Chem. Eur. J., 2013, 19, 530-539.

17. K. Sugahara and T. Mikami, Curr. Opin. Struct. Biol., 2007, 17, 536-545.

18. H. Kawashima, K. Atarashi, M. Hirose, J. Hirose, S. Yamada, K. Sugahara and M. Miyasaka, J. Biol. Chem., 2002, 277, 12921-12930.

19. S. S. Deepa, Y. Umehara, S. Higashiyama, N. Itoh and K. Sugahara, J. Biol. Chem., 2002, 277, 43707-43716.

20. C. D. Nandini, T. Mikami, M. Ohta, N. Itoh, F. Akiyama-Nambu and K. Sugahara, J. Biol. Chem., 2004, 279, 50799-50809.

21. X. F. Bao, S. Nishimura, T. Mikami, S. Yamada, N. Itoh and K. Sugahara, J. Biol. Chem., 2004, 279, 9765-9776.

22. C. Noti, J. L. de Paz, L. Polito and P. H. Seeberger, Chem. Eur. J., 2006, 12, 8664-8686.

23. S. Arungundram, K. Al-Mafraji, J. Asong, F. E. Leach, I. J. Amster, A. Venot, J. E. Turnbull and G. J. Boons, J. Am. Chem. Soc., 2009, 131, 17394-17405.

24. F. Baleux, L. Loureiro-Morais, Y. Hersant, P. Clayette, F. Arenzana-Seisdedos, D. Bonnaffe and H. Lortat-Jacob, Nat. Chem. Biol., 2009, 5, 743-748.

25. J. D. C. Codee, B. Stubba, M. Schiattarella, H. S. Overkleeft, C. A. A. van Boeckel, J. H. van Boom and G. A. van der Marel, J. Am. Chem. Soc., 2005, 127, 3767-3773.

26. P. Czechura, N. Guedes, S. Kopitzki, N. Vazquez, M. Martin-Lomas and N. C. Reichardt, Chem. Commun., 2011, 47, 2390-2392.

27. R. Ojeda, J. L. de Paz and M. Martin-Lomas, Chem. Commun., 2003, 24862487.

28. T. Polat and C. H. Wong, J. Am. Chem. Soc., 2007, 129, 12795-12800.

29. G. Tiruchinapally, Z. Yin, M. El-Dakdouki, Z. Wang and X. Huang, Chem. Eur. J., 2011, 17, 10106-10112.

30. Y.-P. Hu, S.-Y. Lin, C.-Y. Huang, M. M. L. Zulueta, J.-Y. Liu, W. Chang and S.-C. Hung, Nat. Chem., 2011, 3, 557-563.

31. Z. Wang, Y. M. Xu, B. Yang, G. Tiruchinapally, B. Sun, R. P. Liu, S. Dulaney, J. A. Liu and X. F. Huang, Chem. Eur. J., 2010, 16, 8365-8375.

32. C. Lopin and J. C. Jacquinet, Angew. Chem. Int. Ed., 2006, 45, 2574-2578.

33. M. T. C. Walvoort, A. G. Volbeda, N. R. M. Reintjens, H. van den Elst, O. J. Plante, H. S. Overkleeft, G. A. van der Marel and J. D. C. Codee, Org. Lett., 2012, 14, 3776-3779. 
34. A.-R. de Jong, B. Hagen, V. van der Ark, H. S. Overkleeft, J. D. C. Codee and G. A. van der Marel, J. Org. Chem., 2012, 77, 108-125.

35. Y. Zeng, Z. Wang, D. Whitfield and X. Huang, J. Org. Chem., 2008, 73, 79527962.

36. J. L. de Paz, M. Mar Kayser, G. Macchione and P. M. Nieto, Carbohydr. Res., 2010, 345, 565-571.

37. M. Mar Kayser, J. L. de Paz and P. M. Nieto, Eur. J. Org. Chem., 2010, 21382147.

38. P. H. Seeberger, Chem. Soc. Rev., 2008, 37, 19-28.

39. P. H. Seeberger, Carbohydr. Res., 2008, 343, 1889-1896.

40. O. J. Plante, E. R. Palmacci and P. H. Seeberger, Science, 2001, 291, 1523-1527.

41. S. Maza, G. Macchione, R. Ojeda, J. Lopez-Prados, J. Angulo, J. L. de Paz and P. M. Nieto, Org. Biomol. Chem., 2012, 10, 2146-2163.

42. J. L. de Paz, C. Noti and P. H. Seeberger, J. Am. Chem. Soc., 2006, 128, 27662767.

43. J. L. de Paz, E. A. Moseman, C. Noti, L. Polito, U. H. von Andrian and P. H. Seeberger, ACS Chem. Biol., 2007, 2, 735-744.

44. N. Karst and J. C. Jacquinet, Eur. J. Org. Chem., 2002, 815-825.

45. M. Z. Zhao, J. Li, E. Mano, Z. G. Song, D. M. Tschaen, E. J. J. Grabowski and P. J. Reider, J. Org. Chem., 1999, 64, 2564-2566.

46. R. Ojeda, J. L. de Paz, H. Martin-Lomas and J. M. Lassaletta, Synlett, 1999, 1316-1318.

47. J. L. de Paz, R. Ojeda, N. Reichardt and M. Martin-Lomas, Eur. J. Org. Chem., 2003, 3308-3324.

48. J. Tamura, K. W. Neumann and T. Ogawa, Bioorg. Med. Chem. Lett., 1995, 5, $1351-1354$.

49. J. Tamura, K. W. Neumann, S. Kurono and T. Ogawa, Carbohydr. Res., 1998, 305, 43-63.

50. J. Tamura and M. Tokuyoshi, Biosci. Biotechnol. Biochem., 2004, 68, 24362443.

51. J.-i. Tamura, Y. Nakada, K. Taniguchi and M. Yamane, Carbohydr. Res., 2008, 343, 39-47.

52. A. Vibert, C. Lopin-Bon and J.-C. Jacquinet, Eur. J. Org. Chem., 2011, 41834204.

53. J. C. Jacquinet, C. Lopin-Bon and A. Vibert, Chem. Eur. J., 2009, 15, 95799595.

54. A. Vibert, C. Lopin-Bon and J. C. Jacquinet, Chem. Eur. J., 2009, 15, 95619578.

55. S. E. Tully, R. Mabon, C. I. Gama, S. M. Tsai, X. W. Liu and L. C. HsiehWilson, J. Am. Chem. Soc., 2004, 126, 7736-7737.

56. G. Macchione, J. L. de Paz and P. M. Nieto, unpublished work.

57. X. W. Lu, M. N. Kamat, L. J. Huang and X. F. Huang, J. Org. Chem., 2009, 74, 7608-7617.

58. H. Gold, S. Munneke, J. Dinkelaar, H. S. Overkleeft, J. M. F. G. Aerts, J. D. C. Codee and G. A. van der Marel, Carbohydr. Res., 2011, 346, 1467-1478.

59. A. Vibert, C. Lopin-Bon and J.-C. Jacquinet, Tetrahedron Lett., 2010, 51, 18671869.

60. A. F. G. Bongat and A. V. Demchenko, Carbohydr. Res., 2007, 342, 374-406.

61. P. Busca and O. R. Martin, Tetrahedron Lett., 1998, 39, 8101-8104.

62. M. L. Wolfrom and Coniglia.Pj, Carbohydr. Res., 1969, 11, 63-76. 
63. We also carried out this glycosylation with a different GlcA donor [O-(methyl 4$O$-levulinoyl-2,3-di- $O$-pivaloyl- $\alpha, \beta$-D-glucopyranosyluronate) trichloroacetimidate $],{ }^{36,37}$ obtaining the same negative results.

64. J. Dinkelaar, H. Gold, H. S. Overkleeft, J. D. C. Codee and G. A. van der Marel, J. Org. Chem., 2009, 74, 4208-4216.

65. B. Fraser-Reid, J. C. Lopez, A. M. Gomez and C. Uriel, Eur. J. Org. Chem., 2004, 1387-1395.

66. J. Tatai, G. Osztrovszky, M. Kajtar-Peredy and P. Fugedi, Carbohydr. Res., 2008, 343, 596-606.

67. D. Kumagai, M. Miyazaki and S. I. Nishimura, Tetrahedron Lett., 2001, 42, 1953-1956.

68. A. Imamura, A. Kimura, H. Ando, H. Ishida and M. Kiso, Chem. Eur. J., 2006, 12, 8862-8870.

69. F. Belot and J. C. Jacquinet, Carbohydr. Res., 2000, 325, 93-106.

70. S. Maza, J. L. de Paz and P. M. Nieto, Tetrahedron Lett., 2011, 52, 441-443.

71. K. Kakehi, Y. Oda and M. Kinoshita, Anal. Biochem., 2001, 297, 111-116.

72. P. Sorme, B. Kahl-Knutsson, M. Huflejt, U. J. Nilsson and H. Leffler, Anal. Biochem., 2004, 334, 36-47.

73. O. Andrievskaia, Z. Potetinova, A. Balachandran and K. Nielsen, Arch. Biochem. Biophys., 2007, 460, 10-16.

74. E. Gemma, O. Meyer, D. Uhrin and A. N. Hulme, Mol. BioSyst., 2008, 4, 481495.

75. S. Faham, R. E. Hileman, J. R. Fromm, R. J. Linhardt and D. C. Rees, Science, 1996, 271, 1116-1120.

76. R. Raman, V. Sasisekharan and R. Sasisekharan, Chem. Biol., 2005, 12, 267277.

77. S. Maza, J. L. de Paz and P. M. Nieto, unpublished work.

78. K. D. Johnstone, T. Karoli, L. Liu, K. Dredge, E. Copeman, C. P. Li, K. Davis, E. Hammond, I. Bytheway, E. Kostewicz, F. C. K. Chiu, D. M. Shackleford, S. A. Charman, W. N. Charman, J. Harenberg, T. J. Gonda and V. Ferro, J. Med. Chem., 2010, 53, 1686-1699.

79. V. Ferro, L. Liu, K. D. Johnstone, N. Wimmer, T. Karoli, P. Handley, J.

Rowley, K. Dredge, C. P. Li, E. Hammond, K. Davis, L. Sarimaa, J. Harenberg and I. Bytheway, J. Med. Chem., 2012, 55, 3804-3813. 